\title{
Temperature-Dependent Effects of Alkyl Substitution on Diarylamine Reactivity
}

\author{
Ron Shah, Jia-Fei Poon, Evan A. Haidasz and Derek A. Pratt* \\ Department of Chemistry and Biomolecular Sciences, University of Ottawa \\ Ottawa, ON K1N 6N5, Canada \\ dpratt@uottawa.ca
}

Supporting Information

\section{Table of Contents}

I. Computed Structures of Diarylamines and their Diarylaminyl Radicals ..........................S3

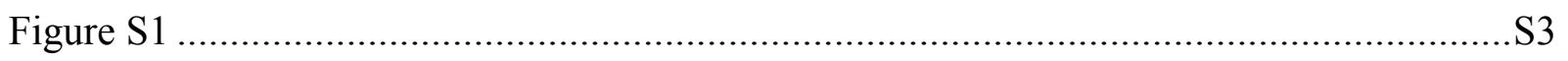

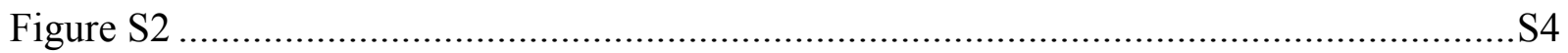

Table S1. Key dihedral angles and calculated N-H BDE ................................................S5

II. Determination of $\alpha 2 \mathrm{H}$ Values of the Diarylamines by ${ }^{1} \mathrm{H}$ NMR ....................................S5

Table S2. Summary of $\alpha 2 H$ values determined by ${ }^{1} \mathrm{H}$ NMR ..........................................S5

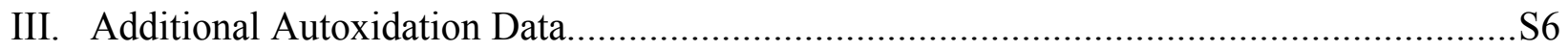

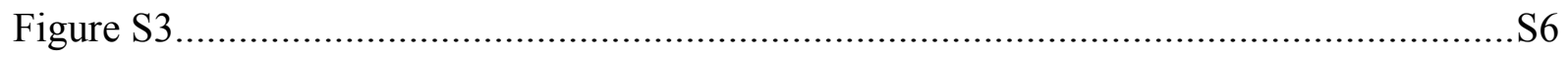

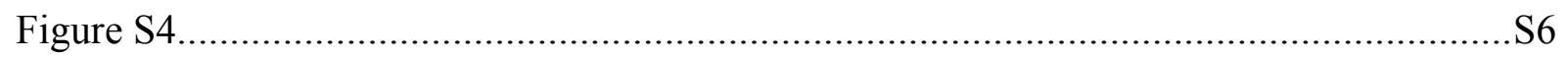

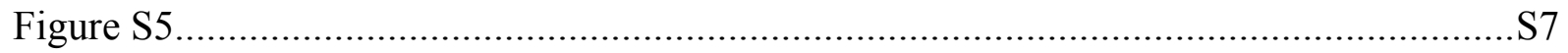

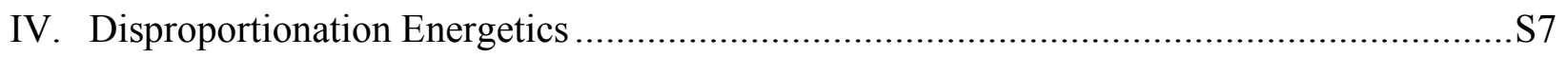

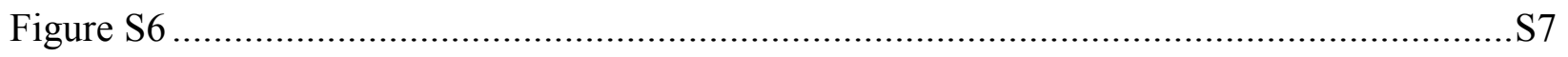

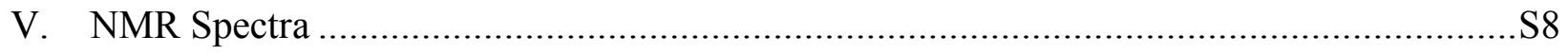

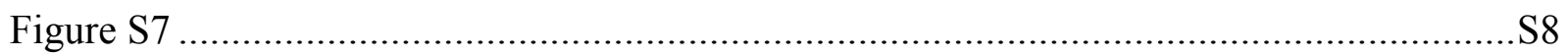

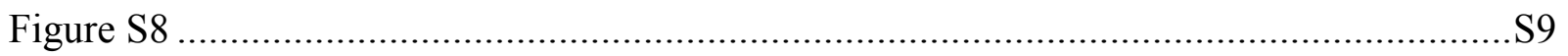

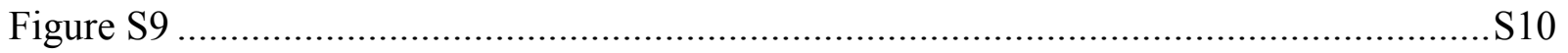

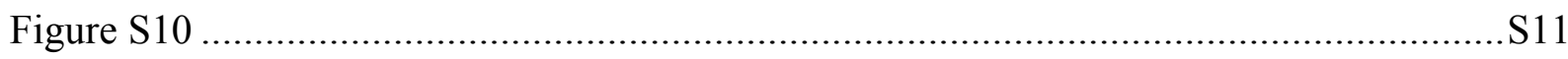

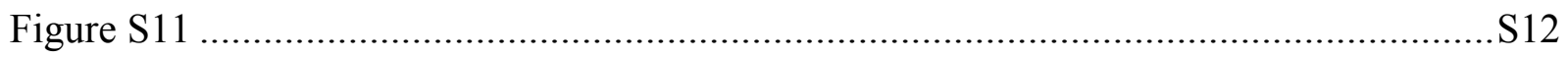

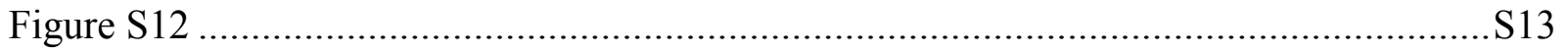

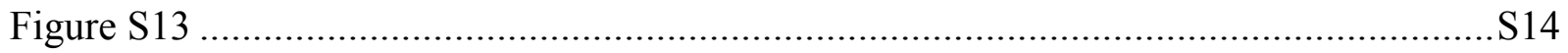

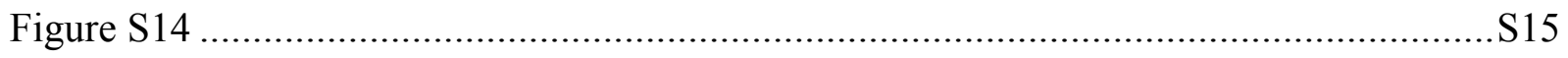

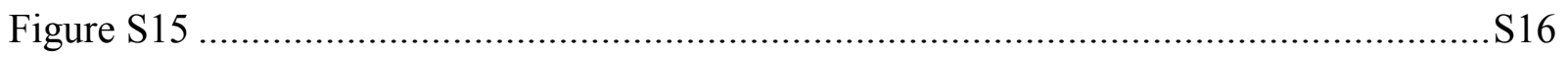

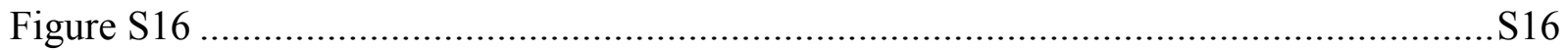

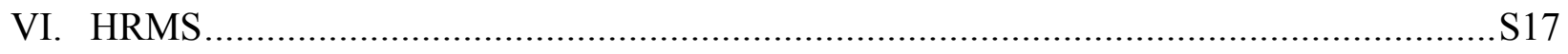

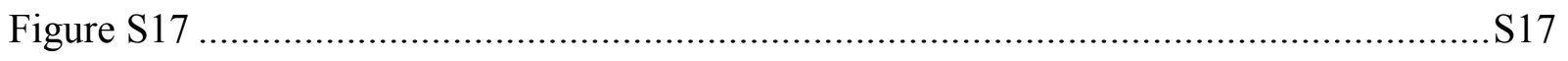




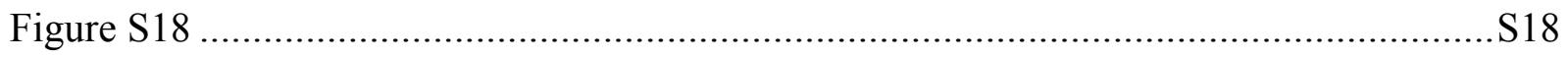

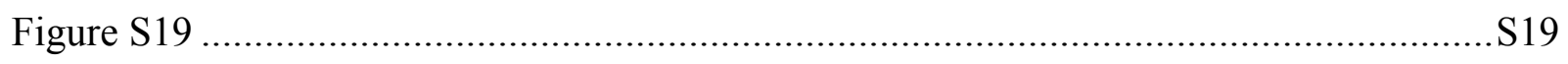

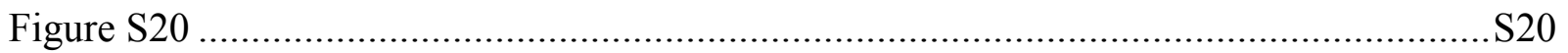

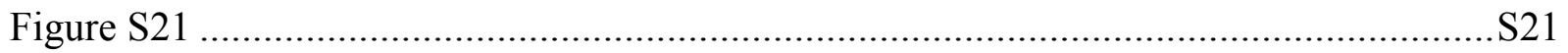

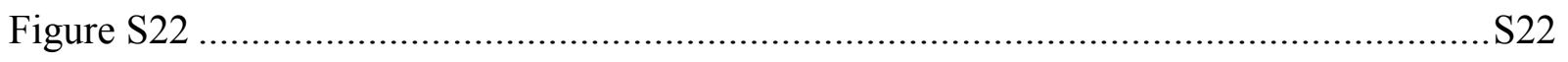

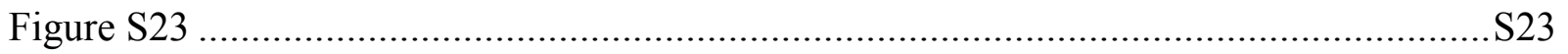

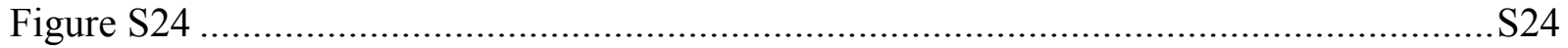

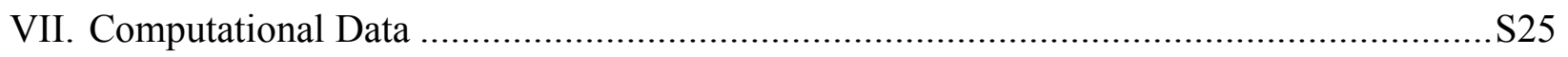


I. Computed Structures of Diarylamines and their Diarylaminyl Radicals

A

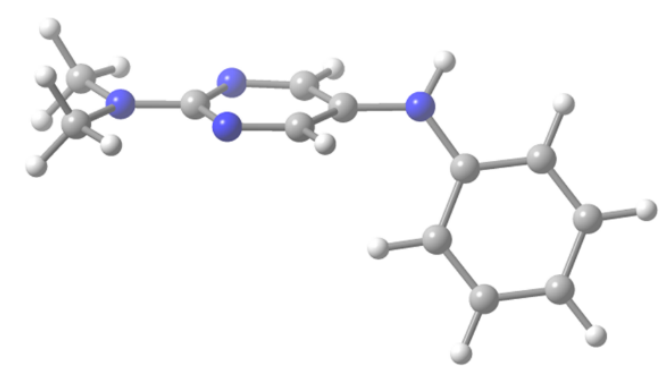

C

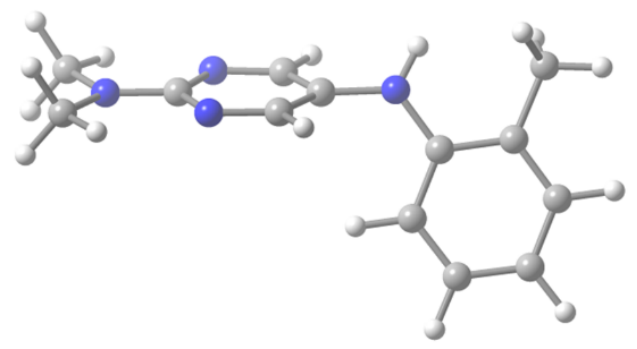

E

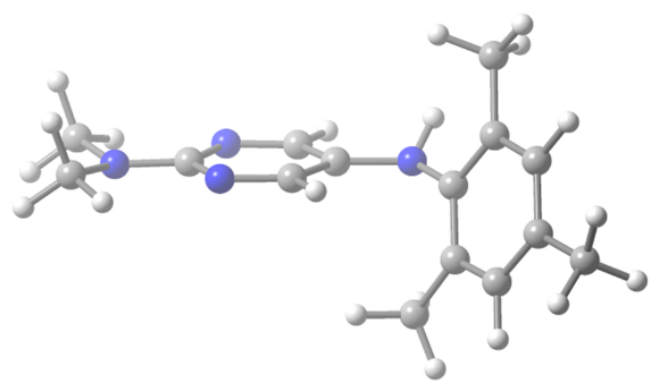

G

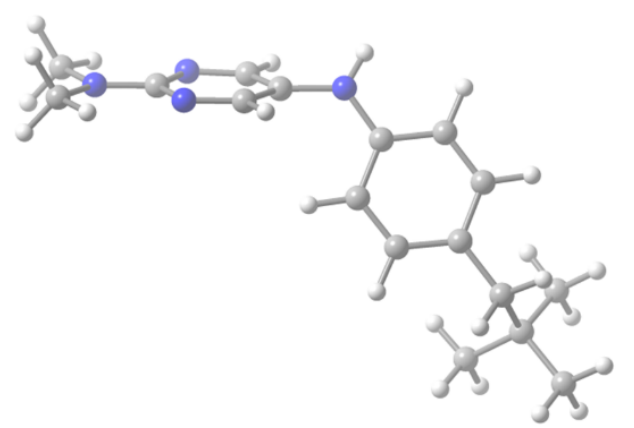

B

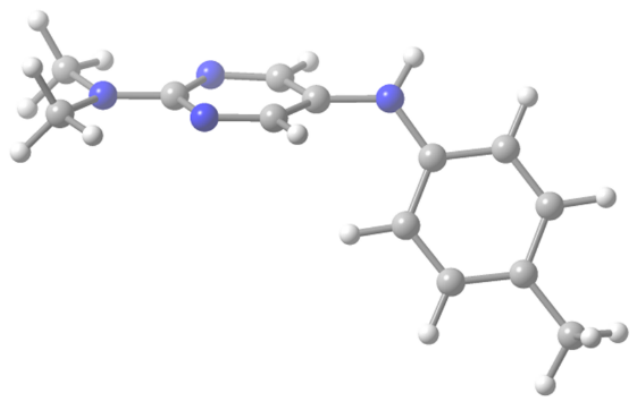

D

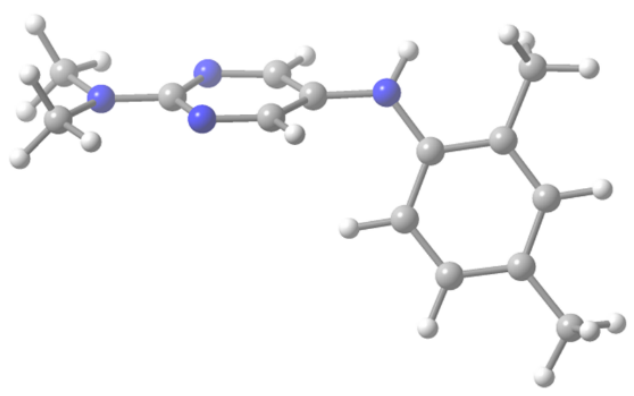

F

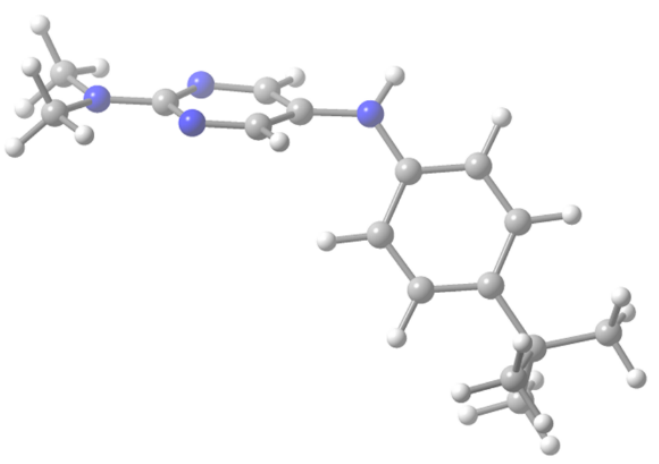

$\mathrm{H}$

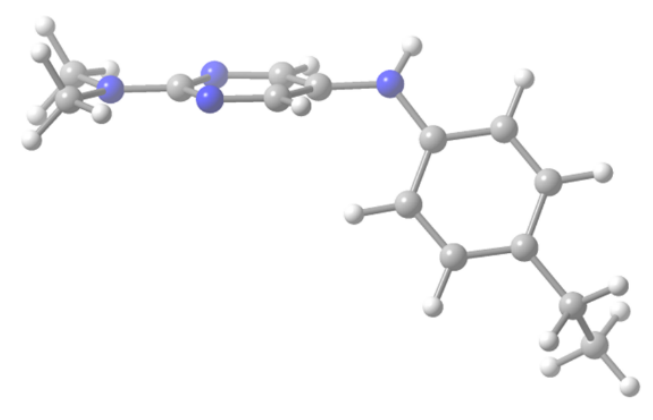

Figure S1. Computed structures of 2 (A), $p$-Me-2 (B), $o$-Me-2 (C), $\mathrm{Me}_{2}-2$ (D), mes-2 (E), $t$-Bu-2 (F), neopent-2 (G) and Et-2 (H). 
A

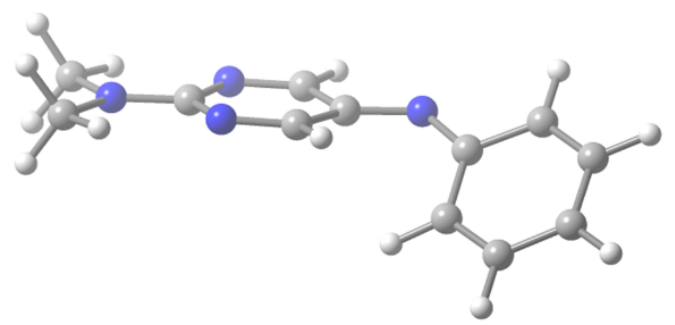

C

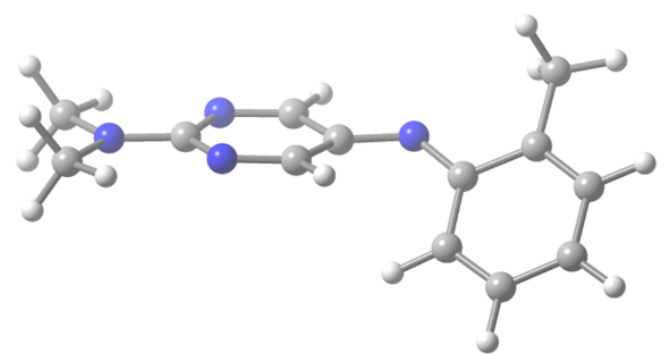

$E$

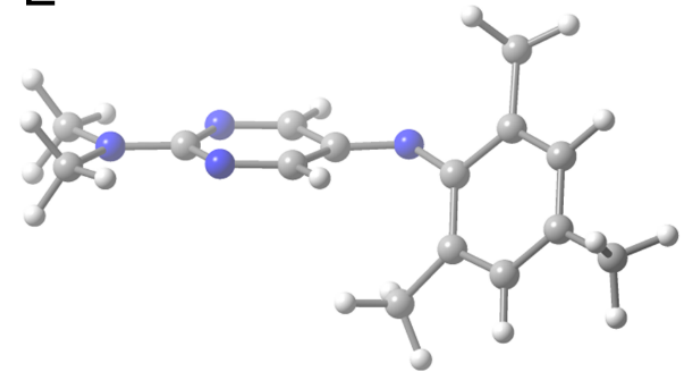

G

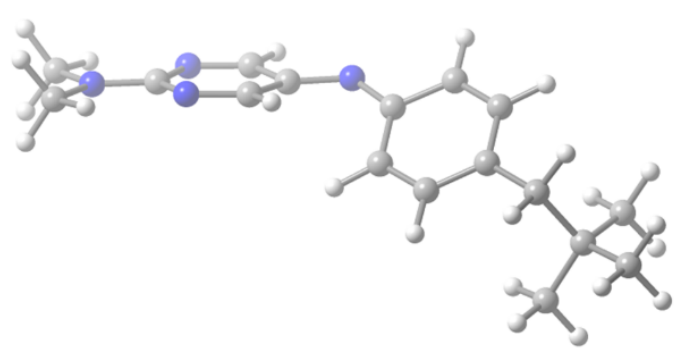

B

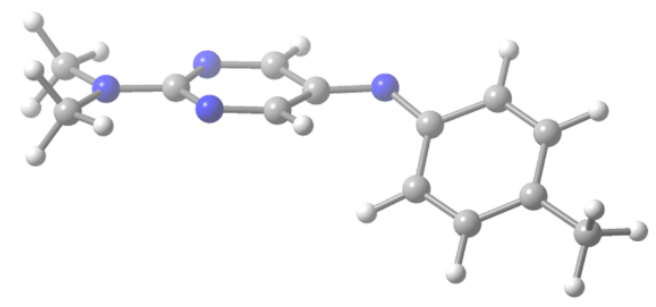

D

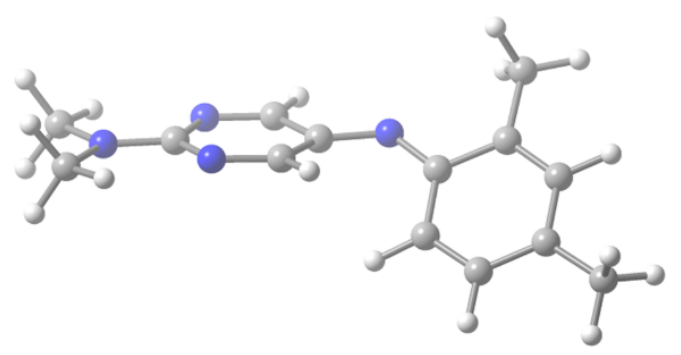

F

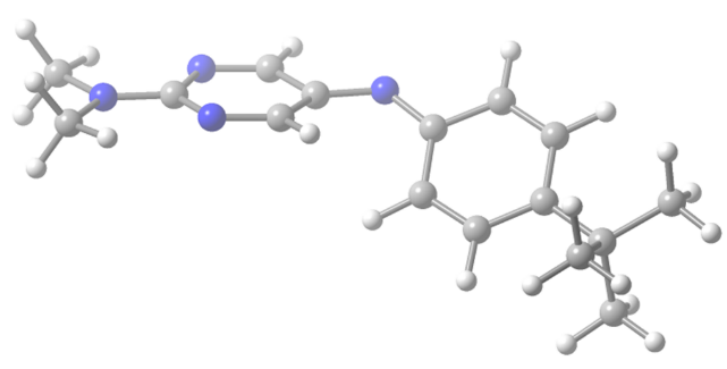

$\mathrm{H}$

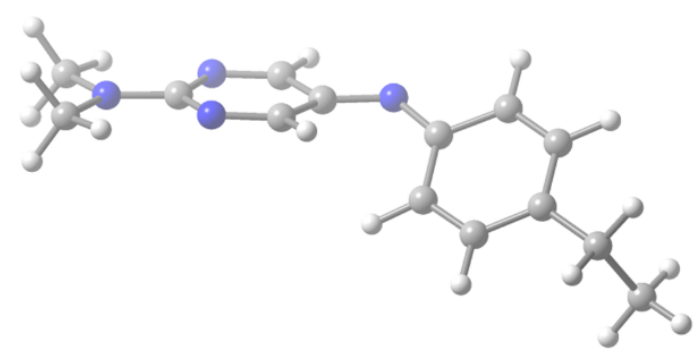

Figure S2. Computed structures of the aminyl radical derived from 2 (A), $p$-Me-2 (B), $o$-Me-2 (C), $\mathrm{Me}_{2}-\mathbf{2}$ (D), mes-2 (E), $t$-Bu-2 (F), neopent-2 (G) and Et-2 (H). 
Table S1. Key dihedral angles and calculated N-H BDEs.

\begin{tabular}{ccccc}
\hline Compound & \multicolumn{2}{c}{ Dihedral angle between the rings } & \multicolumn{2}{c}{$\begin{array}{c}\text { Calculated N-H BDE } \\
\text { (kcal/mol) }\end{array}$} \\
& Amine & Aminyl radical & B3LYP/CBSB7 & CBS-QB3 \\
\hline $\mathbf{2}$ & $55.2^{\circ}$ & $42.7^{\circ}$ & 77.1 & 81.8 \\
$p$-Me-2 & $53.5^{\circ}$ & $41.5^{\circ}$ & 76.4 & 81.3 \\
$o-\mathrm{Me}-2$ & $57.5^{\circ}$ & $44.4^{\circ}$ & 76.3 & 81.8 \\
$\mathrm{Me}_{2}-2$ & $56.4^{\circ}$ & $43.3^{\circ}$ & 75.5 & 81.2 \\
mes-2 & $64.3^{\circ}$ & $58.1^{\circ}$ & 76.9 & 82.7 \\
$t$-Bu-2 & $53.0^{\circ}$ & $41.4^{\circ}$ & 76.6 & $\mathrm{nd}^{\mathrm{a}}$ \\
neopent-2 & $54.2^{\circ}$ & $41.6^{\circ}$ & 76.6 & $\mathrm{nd}^{\mathrm{a}}$ \\
Et-2 & $54.0^{\circ}$ & $41.6^{\circ}$ & 76.5 & 81.4 \\
\hline
\end{tabular}

${ }^{\mathrm{a}}$ Not determined. CBS-QB3 calculations were beyond the current limit of our computational resources.

\section{Determination of $\alpha_{2}^{H}$ Values of the Diarylamines by ${ }^{1} \mathrm{H}$ NMR}

Functional groups which engage significantly as hydrogen bond donors (having measurable $\alpha_{2}^{H}$ values) demonstrate substantially lower-field chemical shifts in a strongly hydrogen bonding solvent (DMSO-d6) compared to a weakly hydrogen bonding solvent $\left(\mathrm{CDCl}_{3}\right.$ or Benzene- $\left.\mathrm{d}_{6}\right)$. The correlation between $\alpha_{2}^{H}$ values and the $\Delta \delta$ of DMSO-d6 and $\mathrm{CDCl}_{3}$ was described by Abraham et al to afford the empirical relationship Eq. S1. In a previously published paper we utilized benzene-d6 instead of $\mathrm{CDCl}_{3}$ (due to an unusual incompatibility between $\mathrm{CDCl}_{3}$ and several of our phenoxazines/phenothiazines) which afforded a similar correlation rendering Eq. S2.

$\alpha_{2}^{H}=0.133 \Delta \delta_{D M S O-C D C l 3}+0.0066 \quad$ Eq. $\mathrm{S} 1$

$\alpha_{2}^{H}=0.134 \Delta \delta_{D M S O-B e n}-0.1087 \quad$ Eq. S2

Table S2. Summary of $\boldsymbol{\alpha}_{\mathbf{2}}^{\boldsymbol{H}}$ values determined by ${ }^{1} \mathrm{H}$ NMR

\begin{tabular}{|c|c|c|c|c|}
\hline & $\begin{array}{c}\text { SBenzene-d6 } \\
(\mathrm{ppm})\end{array}$ & $\begin{array}{c}\text { SDMSO-d6 } \\
(\mathrm{ppm})\end{array}$ & $\Delta \delta_{\text {DMSO-Ben }}(\mathbf{p p m})$ & $\begin{array}{c}\text { Calculated } \alpha_{2}^{H} \\
\left({ }^{1} \mathrm{H} \text { NMR }\right)\end{array}$ \\
\hline 2 & 4.18 & 7.52 & 3.34 & 0.33 \\
\hline$t-\mathrm{Bu}-2$ & 4.21 & 7.35 & 3.14 & 0.31 \\
\hline neopent-2 & 4.20 & 7.40 & 3.20 & 0.32 \\
\hline$p$-Me-2 & 4.16 & 7.36 & 3.20 & 0.32 \\
\hline$o-\mathrm{Me}-2$ & 4.25 & 6.71 & 2.46 & 0.32 \\
\hline $\mathrm{Me}_{2}-2$ & 4.18 & 6.59 & 2.41 & 0.21 \\
\hline mest-2 & 3.93 & 6.62 & 2.69 & 0.25 \\
\hline 4 & 4.93 & 7.82 & 2.89 & 0.28 \\
\hline 5 & 4.92 & 7.82 & 2.90 & 0.28 \\
\hline
\end{tabular}




\section{Additional Autoxidation Data}
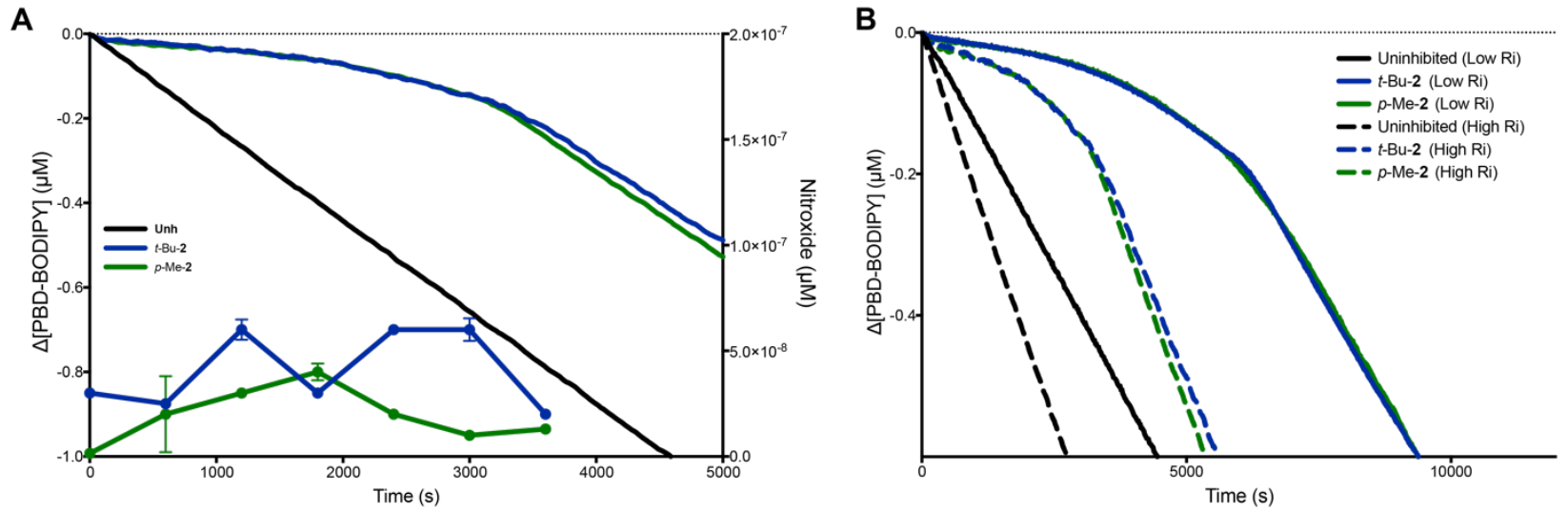

Figure S3. (A) Nitroxide formation in styrene autoxidations for $t$-Bu-2 and $p-\mathrm{Me}-2$ overlaid with their inhibition activity. Co-autoxidation of styrene (4.3 M) and PBD-BODIPY $(10 \mu \mathrm{M})$ initiated by AIBN (3 $\mathrm{mM}$ or $6 \mathrm{mM})$ in $\mathrm{PhCl}$ at $37^{\circ} \mathrm{C}$, monitored at $591 \mathrm{~nm}\left(\varepsilon=139,000 \mathrm{M}^{-1} \mathrm{~cm}^{-1}\right)$. Uninhibited (black), or inhibited with $2 \mu \mathrm{M}$ of $t$-Bu-2 or $p$-Me-2. Data obtained with $6 \mathrm{mM}$ AIBN is denoted in dashed lines, while corresponding data with $3 \mathrm{mM}$ AIBN is shown with solid lines.
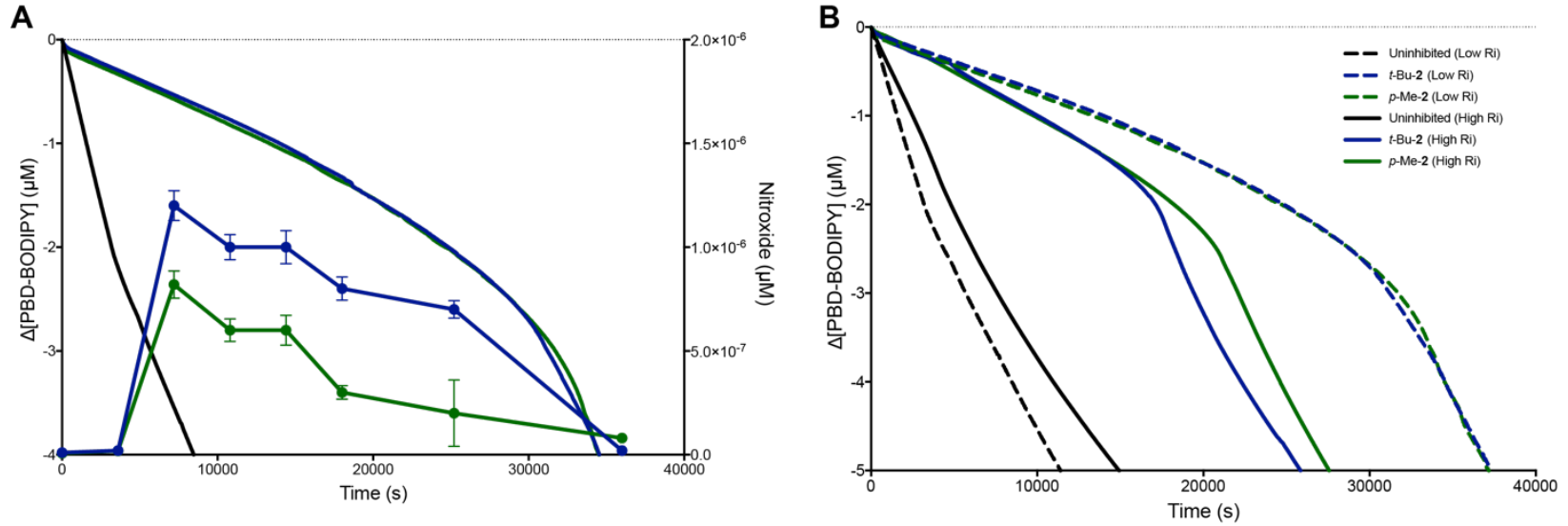

Figure S4. (A) Concentration of $\mathrm{Ar}_{2} \mathrm{NO}$ determined by EPR during $t$-Bu-2 and $p$-Me-2 inhibited coautoxidations of 1-hexadecene. (B) Co-autoxidation of 1-hexadecene (2.8 M) and PBD-BODIPY $(10 \mu \mathrm{M})$ initiated by dicumyl peroxide $(1 \mathrm{mM}$ or $2 \mathrm{mM})$ in $\mathrm{PhCl}$ at $100{ }^{\circ} \mathrm{C}$, monitored at $587 \mathrm{~nm}\left(\varepsilon=131,972 \mathrm{M}^{-}\right.$ $\left.{ }^{1} \mathrm{~cm}^{-1}\right)$. Uninhibited (black), or inhibited with $2 \mu \mathrm{M}$ of $t$-Bu-2 or $p$-Me-2. Data obtained with $1 \mathrm{mM}$ dicumylperoxide is denoted in dashed lines, while corresponding data with $2 \mathrm{mM}$ dicumylperoxide is shown with solid lines. 

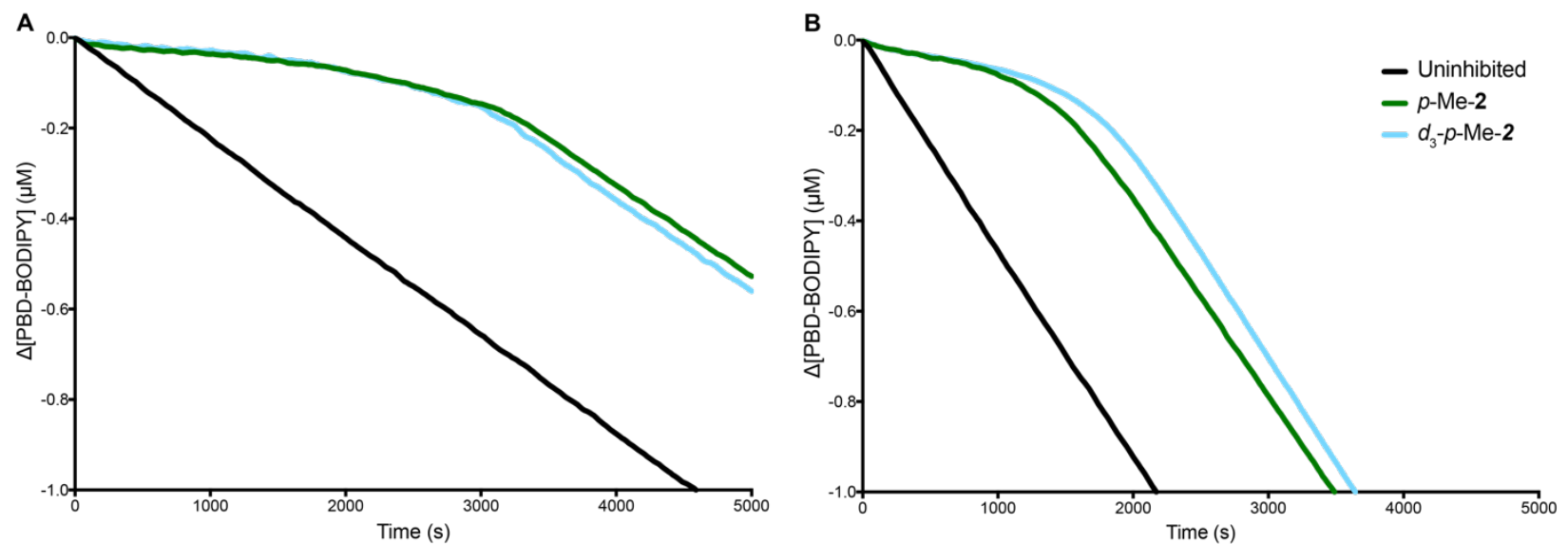

Figure S5. (A) Co-autoxidation of styrene (4.3 M) and PBD-BODIPY $(10 \mu \mathrm{M})$ initiated by AIBN $(6 \mathrm{mM})$ in $\mathrm{PhCl}$ at $37{ }^{\circ} \mathrm{C}$, monitored at $591 \mathrm{~nm}\left(\varepsilon=139,000 \mathrm{M}^{-1} \mathrm{~cm}^{-1}\right)$. Uninhibited (black), or inhibited with $2 \mu \mathrm{M}$ of $p$-Me-2 or $d_{3}-p$-Me-2. (B) Co-autoxidation of 1-hexadecene $(2.8 \mathrm{M})$ and PBD-BODIPY $(10 \mu \mathrm{M})$ initiated by dicumyl peroxide $(1 \mathrm{mM})$ in $\mathrm{PhCl}$ at $100^{\circ} \mathrm{C}$, monitored at $587 \mathrm{~nm}\left(\varepsilon=131,972 \mathrm{M}^{-1} \mathrm{~cm}^{-1}\right)$. Uninhibited (black), or inhibited with $2 \mu \mathrm{M}$ of $p$-Me-2 or $d_{3}-p$-Me-2.

\section{Disproportionation Energetics}<smiles>Cc1ccc(N([O-])c2ccccc2)cc1</smiles><smiles>C=C1C=CC([N+]([O-])=C2C=CC=CC2)C=C1</smiles>

$\Delta \mathrm{G}=4.0 \mathrm{kcal} / \mathrm{mol}$

2<smiles>Cc1ccc(Nc2ccccc2)cc1</smiles>

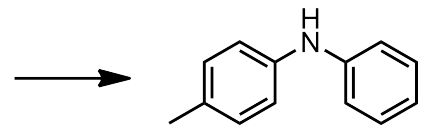<smiles>C=C1C=CC(=Nc2ccccc2)C=C1</smiles>

$\Delta \mathrm{G}=-21.0 \mathrm{kcal} / \mathrm{mol}$<smiles>C=C1C=CC([N+]([O-])(O)c2cnc(NC(C)C)nc2)C=C1</smiles>

$\Delta \mathrm{G}=9.9 \mathrm{kcal} / \mathrm{mol}$<smiles>C=C1C=CC(=Nc2cnc(NC(C)=O)nc2)C=C1</smiles>

$\Delta \mathrm{G}=-14.5 \mathrm{kcal} / \mathrm{mol}$

Figure S6. CBS-QB3-computed free energies changes for the disproportionation of nitroxides and aminyl radicals derived from ditolylamine and $p$-Me-2. 
V. NMR Spectra
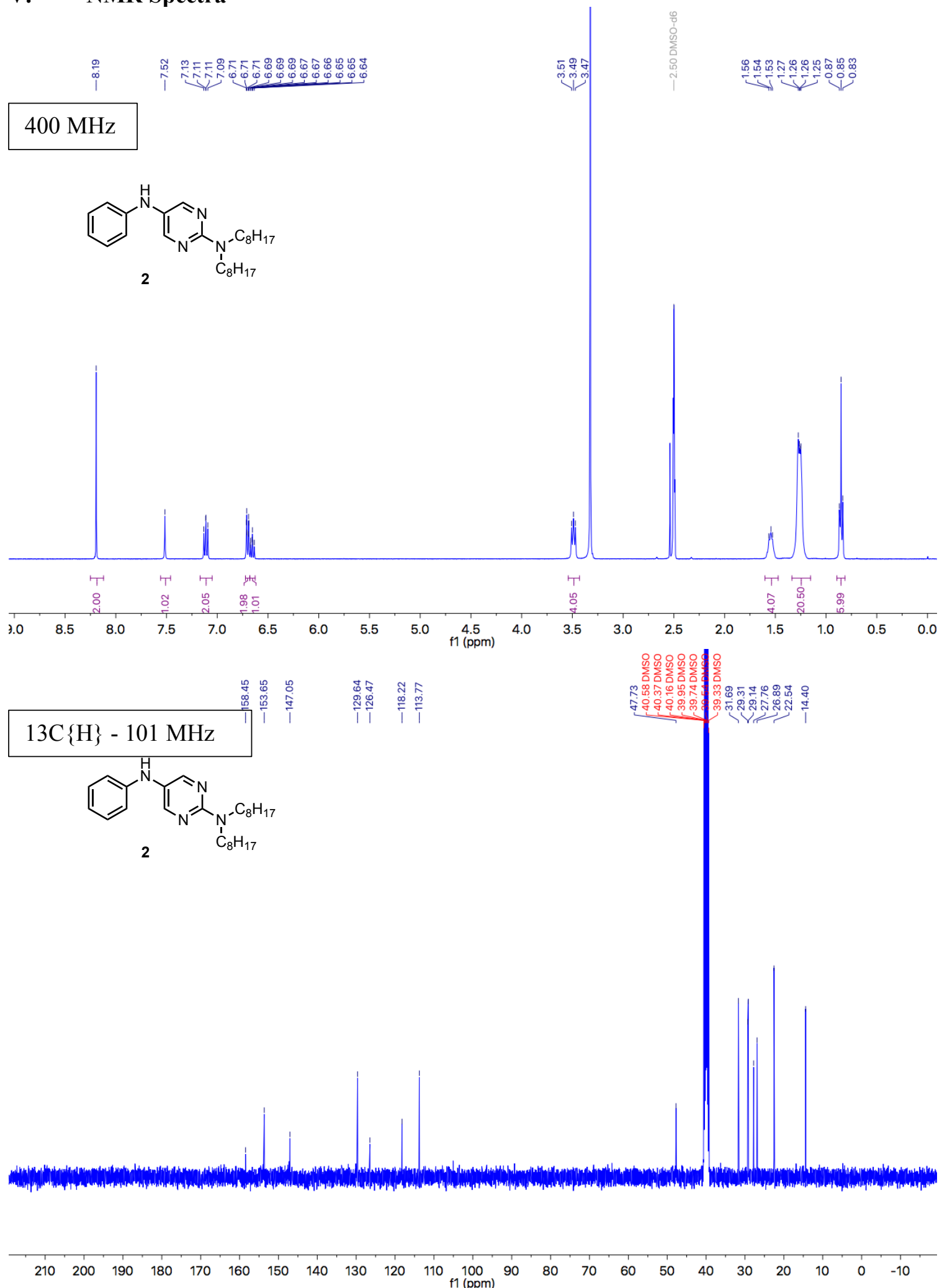

Figure S7. ${ }^{1} \mathrm{H}$ NMR and ${ }^{13} \mathrm{C}\{\mathrm{H}\}$ NMR of 2 

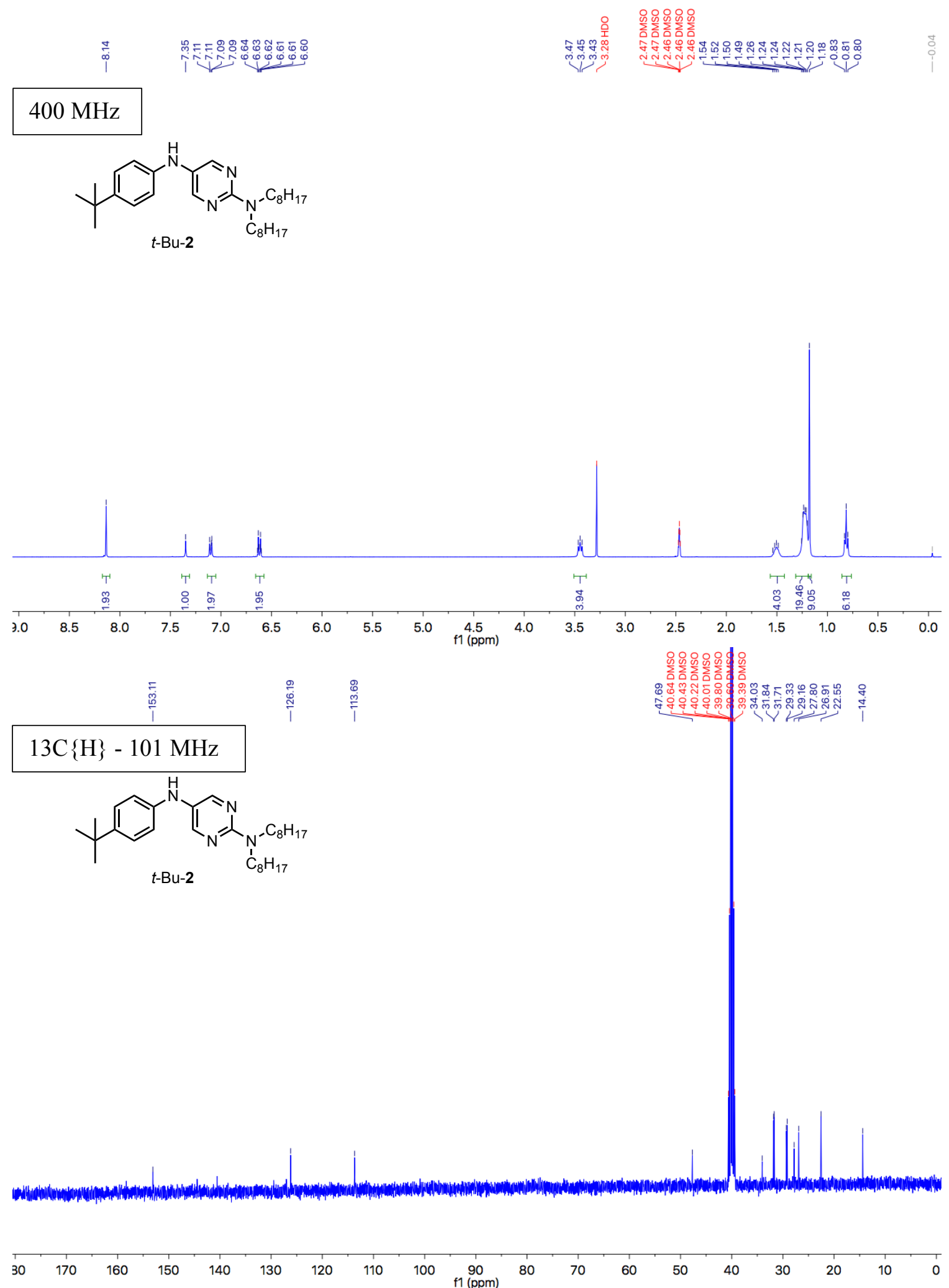

Figure S8. ${ }^{1} \mathrm{H}$ NMR and ${ }^{13} \mathrm{C}\{\mathrm{H}\}$ NMR of $t$-Bu-2 

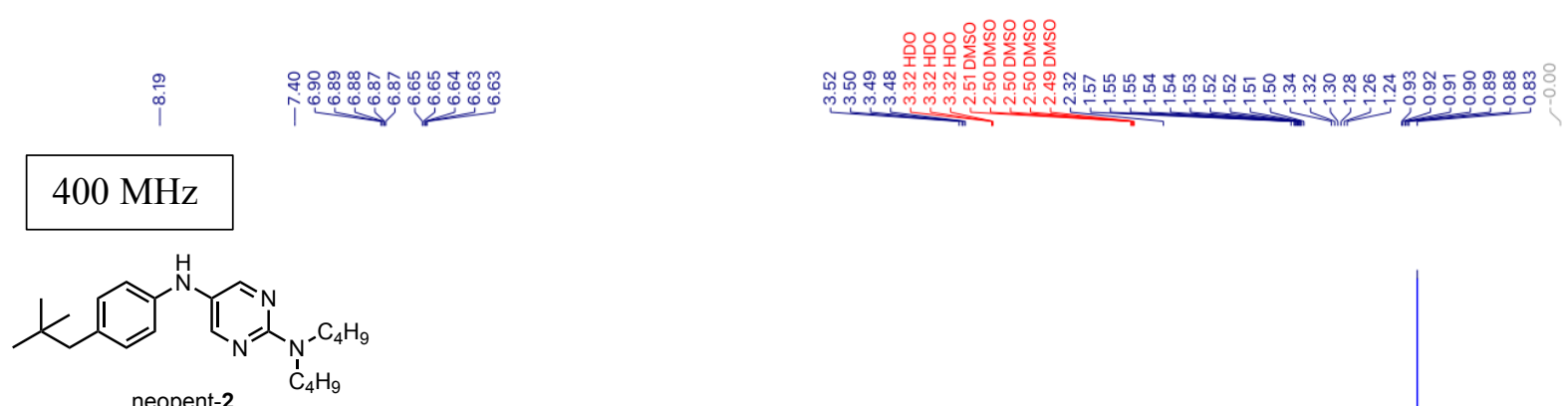

neopent-2
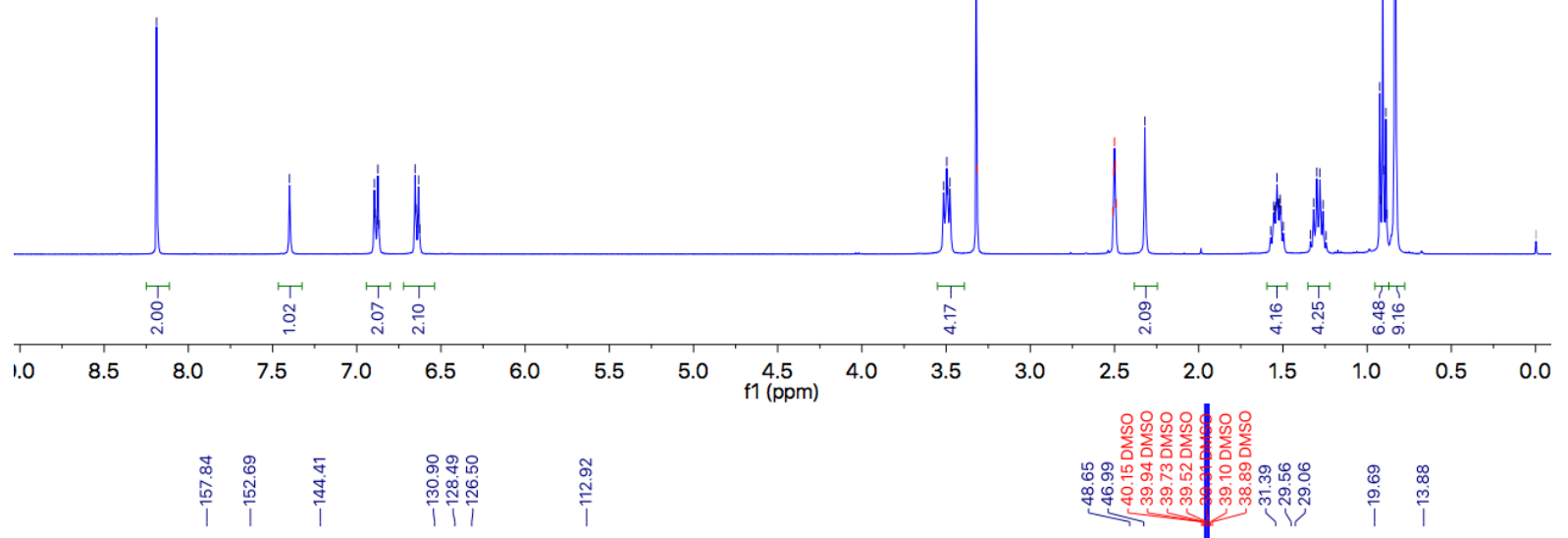

$\stackrel{\text { ָ̃ }}{\stackrel{\text { I }}{\nu}}$

$13 \mathrm{C}\{\mathrm{H}\}-101 \mathrm{MHz}$

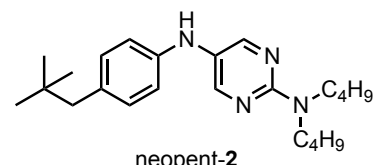

neopent-2
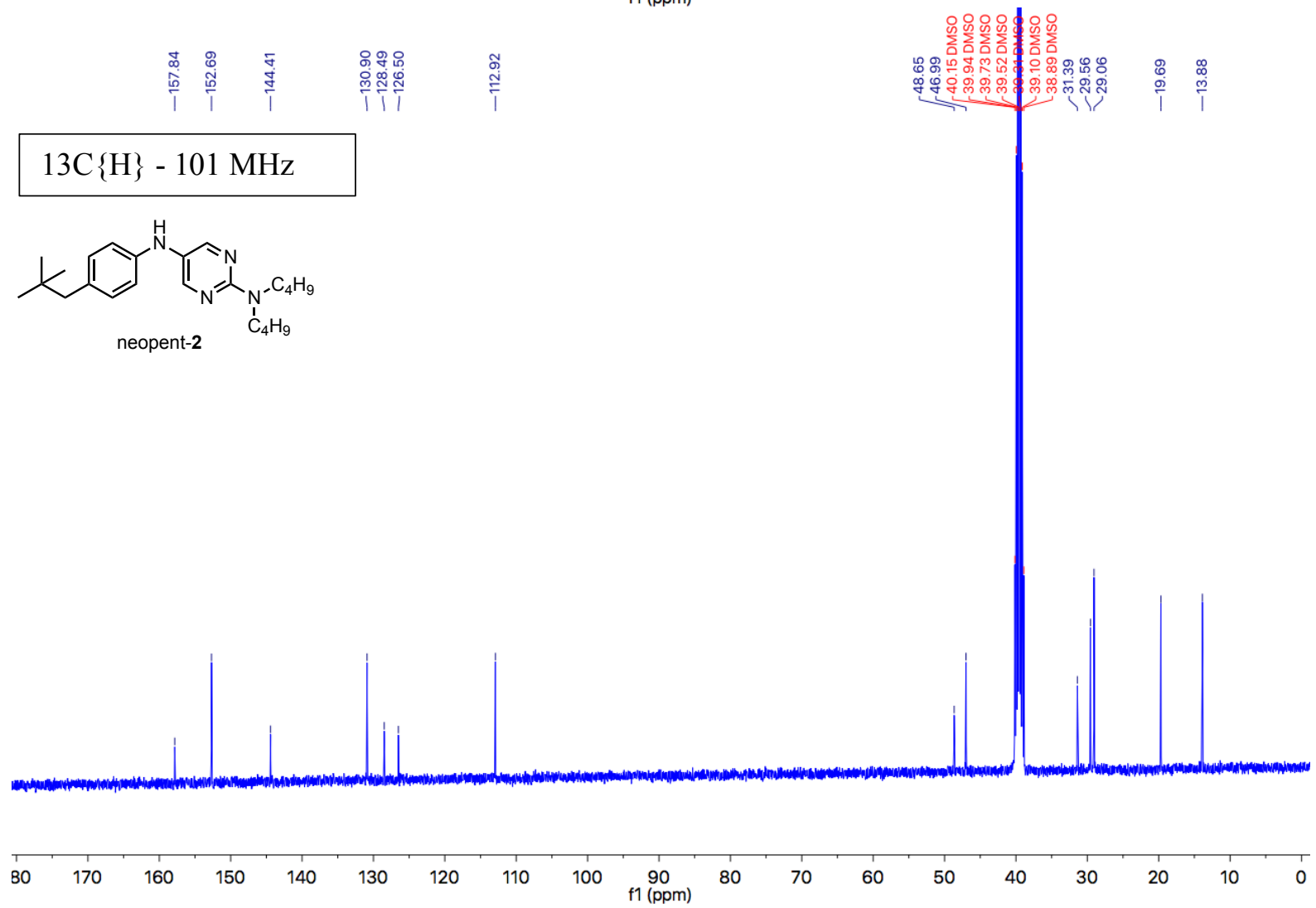

Figure S9. ${ }^{1} \mathrm{H}$ NMR and ${ }^{13} \mathrm{C}\{\mathrm{H}\}$ NMR of neopent-2

S10 
<smiles>CCCN(CC)c1ncc(Nc2ccc(C)cc2)cn1</smiles>

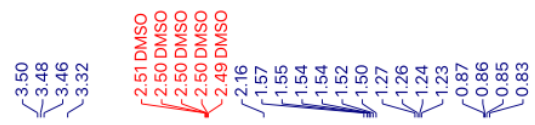

$\stackrel{\circ}{\circ}$

$400 \mathrm{MHz}$

p-Me-2
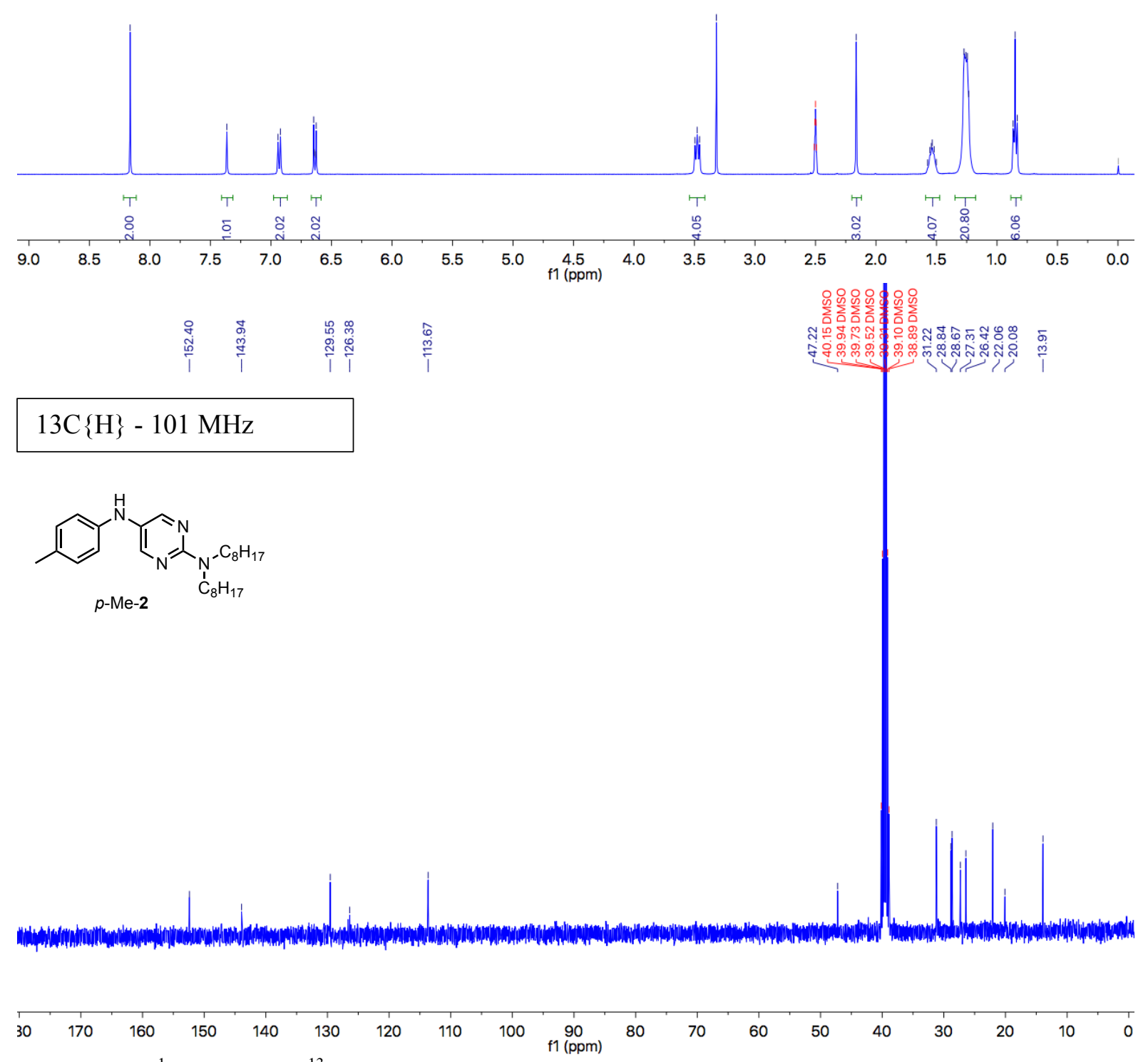

Figure S10. ${ }^{1} \mathrm{H}$ NMR and ${ }^{13} \mathrm{C}\{\mathrm{H}\}$ NMR of $p$-Me-2

Si 1 


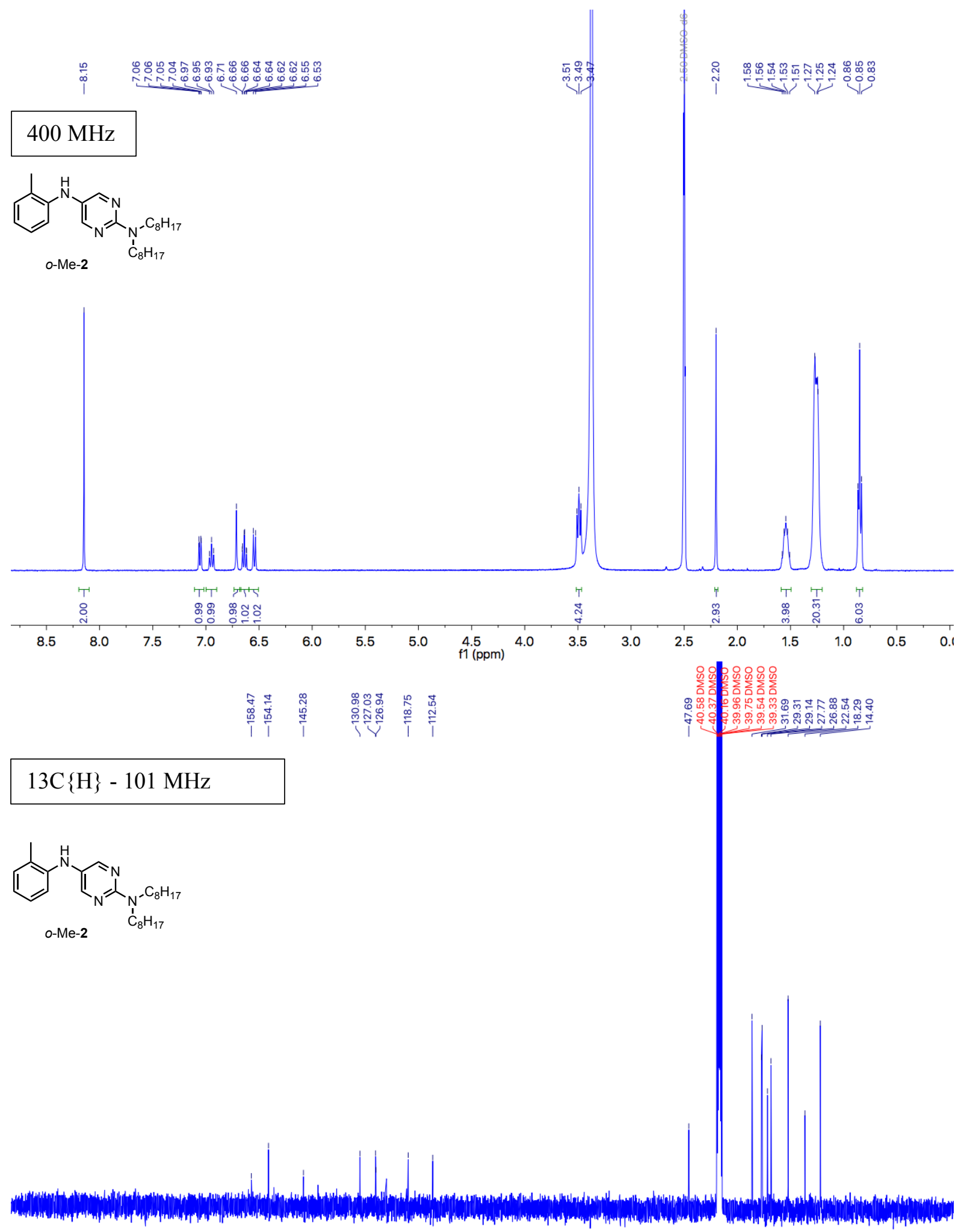

$\begin{array}{llllllllllllllllllllllllll}210 & 200 & 190 & 180 & 170 & 160 & 150 & 140 & 130 & 120 & 110 & \begin{array}{c}100 \\ \mathrm{f}(\mathrm{ppm})\end{array} & 90 & 80 & 70 & 60 & 50 & 40 & 30 & 20 & 10 & 0 & -10 & \end{array}$

Figure S11. ${ }^{1} \mathrm{H} \mathrm{NMR}$ and ${ }^{13} \mathrm{C}\{\mathrm{H}\} \mathrm{NMR}$ of $o-\mathrm{Me}-2$ 

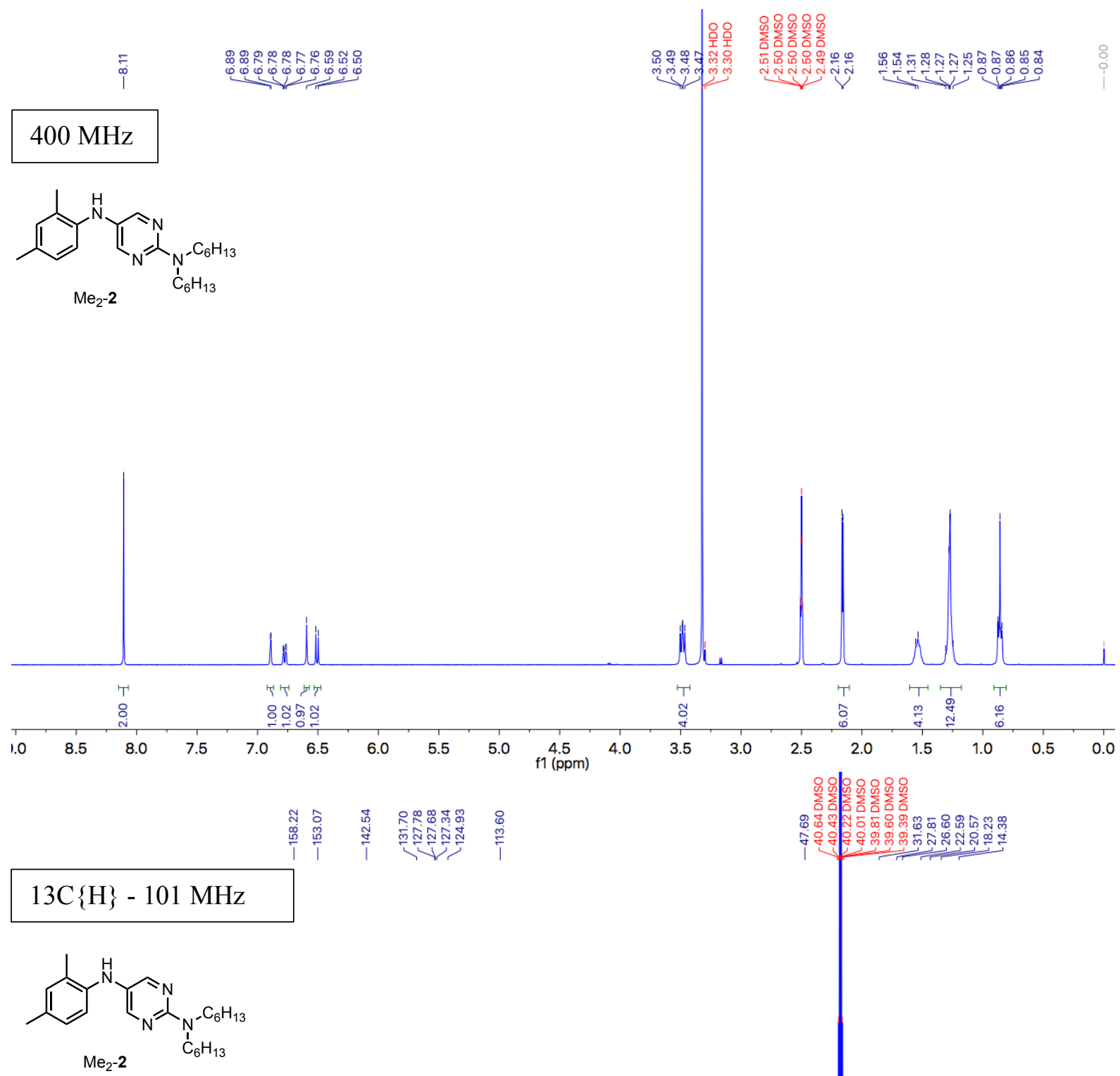

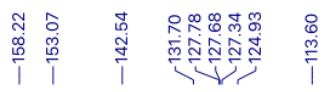

๑ ษ ๆ

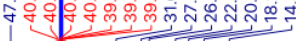

$\mathrm{C}_{6} \mathrm{H}_{13}$
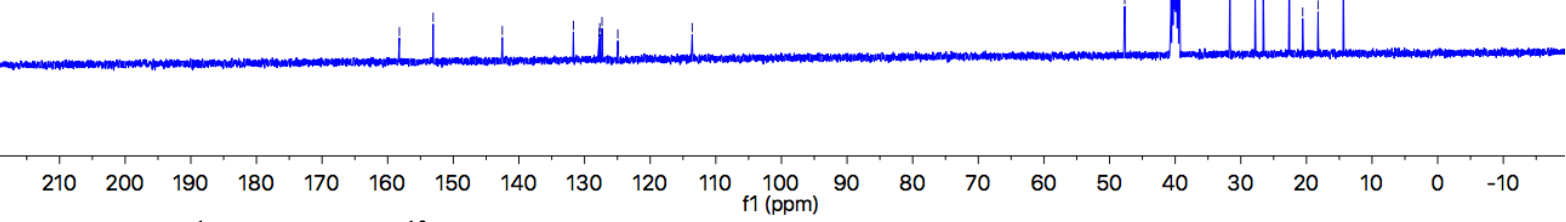

Figure S12. ${ }^{1} \mathrm{H} \mathrm{NMR}$ and ${ }^{13} \mathrm{C}\{\mathrm{H}\} \mathrm{NMR}$ of $\mathrm{Me}_{2}-2$ 
$400 \mathrm{MHz}$

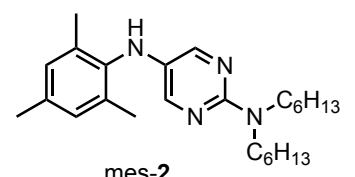

mes-2

$\begin{array}{lll}\overline{0} & \infty & 0 \\ i & 0 & 0 \\ & 1 & 1\end{array}$

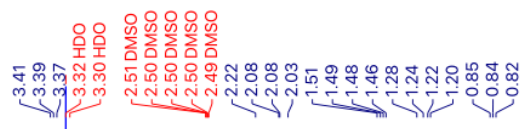

¿
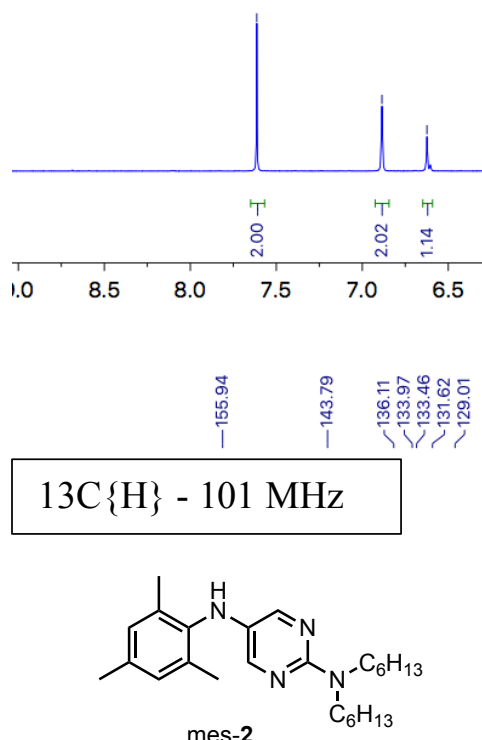

mes-2
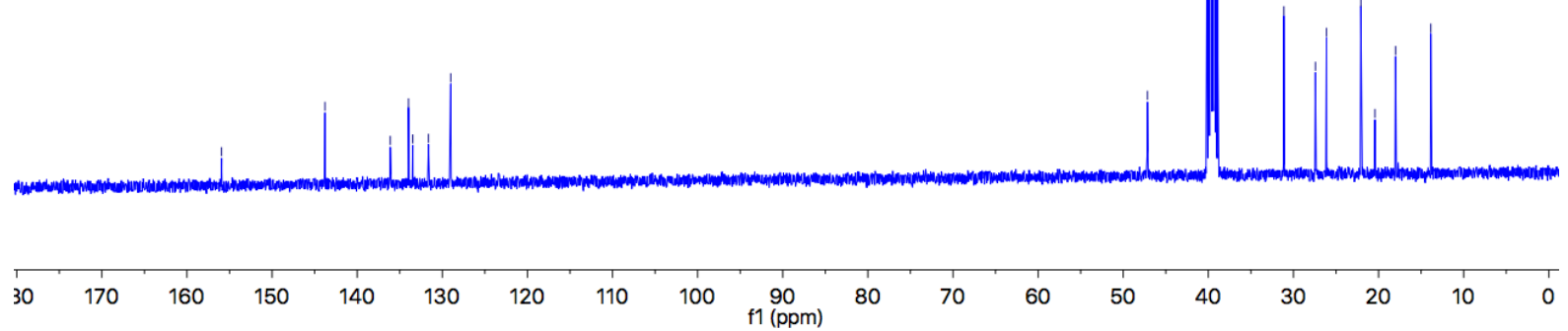

Figure S13. ${ }^{1} \mathrm{H} N \mathrm{NM}$ and ${ }^{13} \mathrm{C}\{\mathrm{H}\}$ NMR of mes-2

S14 

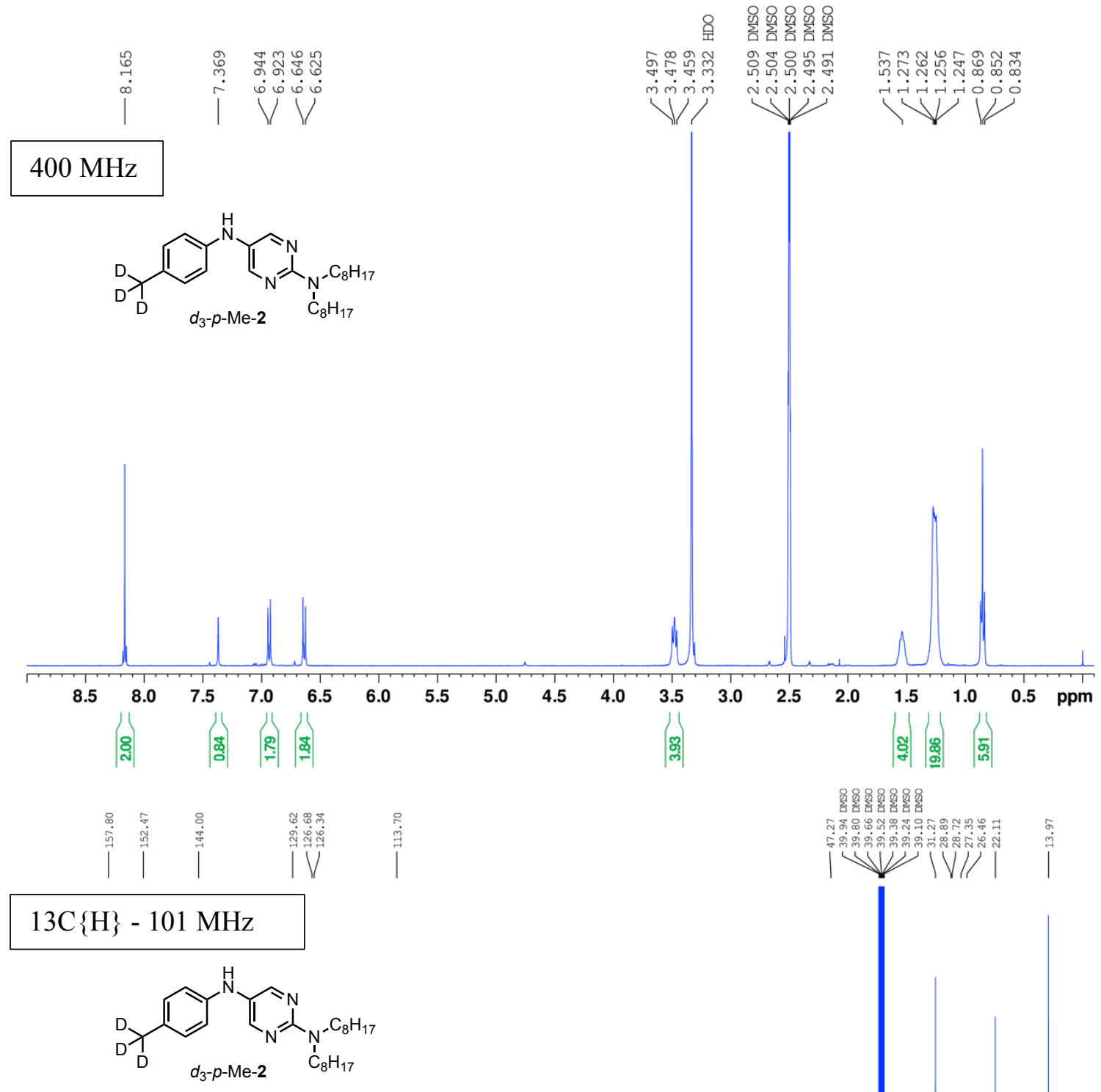

\section{$\stackrel{?}{\stackrel{9}{7}}$}

$13 \mathrm{C}\{\mathrm{H}\}-101 \mathrm{MHz}$

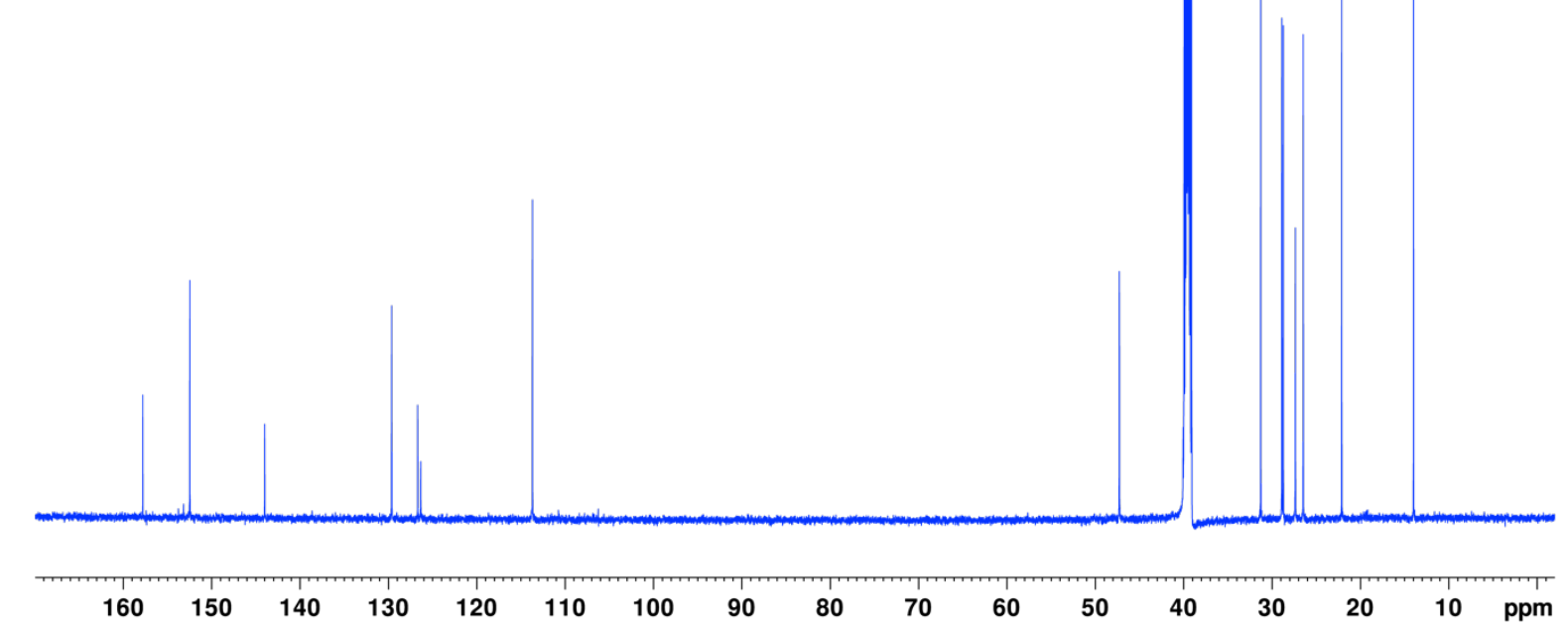

Figure S14. ${ }^{1} \mathrm{H}$ NMR and ${ }^{13} \mathrm{C}\{\mathrm{H}\}$ NMR of $d_{3}-p$-Me-2 


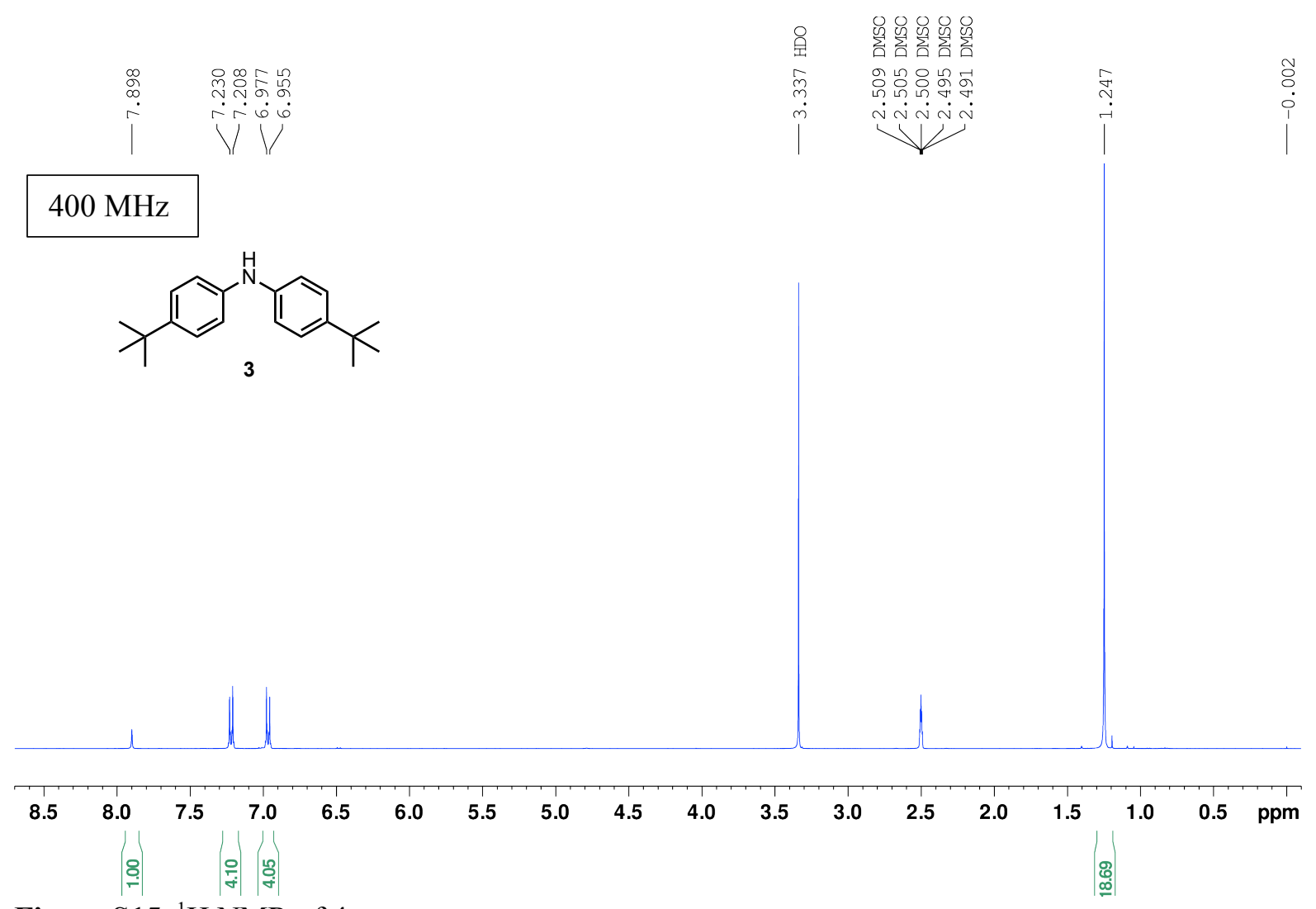

Figure S15. ${ }^{1} \mathrm{H}$ NMR of 4

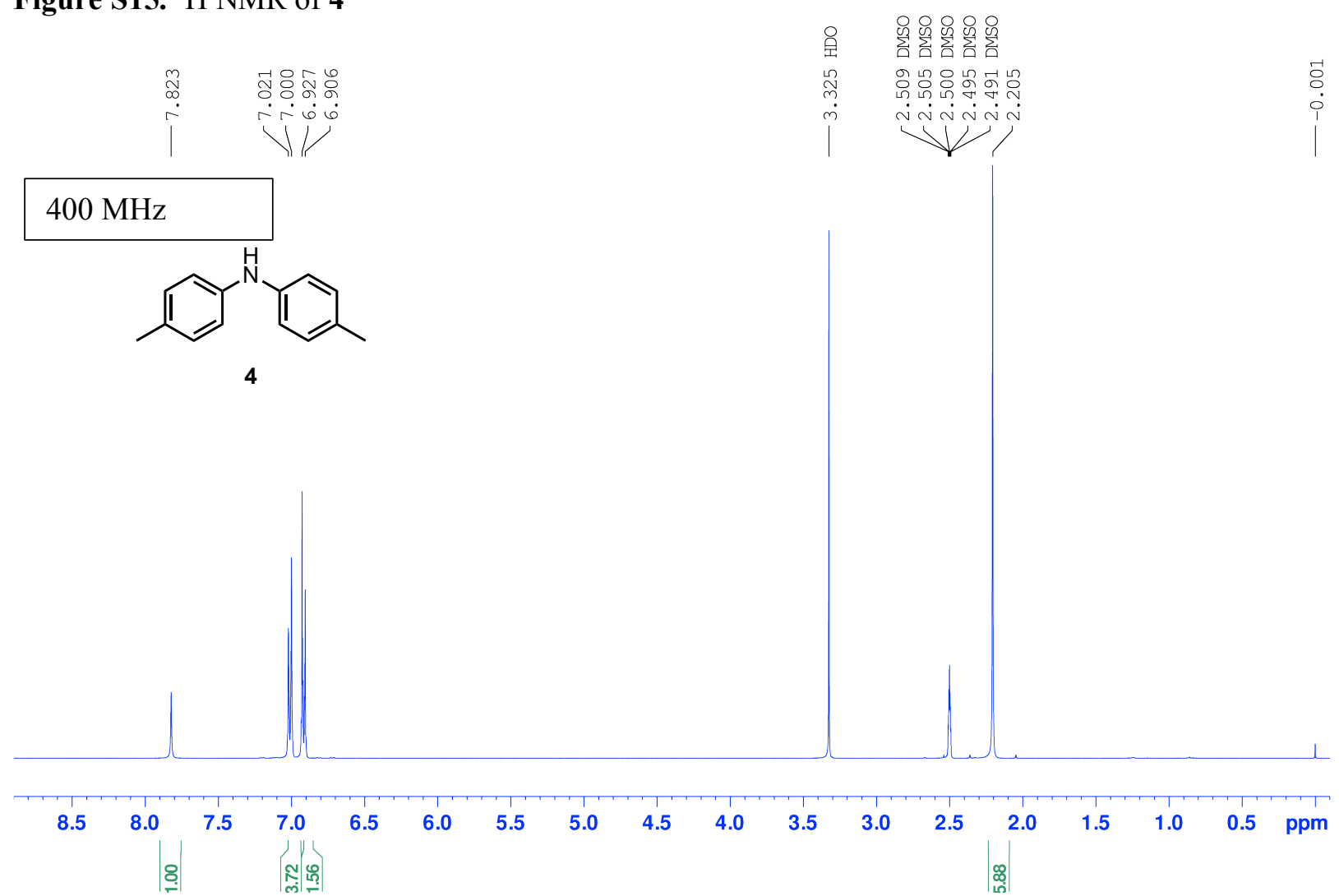

Figure S16. ${ }^{1} \mathrm{H}$ NMR of 5 


\section{HRMS Data}

\begin{tabular}{crrrrr} 
Formula search on scan 2 & of 200600 \\
& & Dev & 12 & 1 & 14 \\
\multirow{2}{*}{ Mass } & Int? & memu & C & H & N \\
& & & & & \\
411.34409 & 1.06 & -4.68 & 26 & 43 & 4 \\
410.33906 & 4.11 & -1.89 & 26 & 42 & 4
\end{tabular}

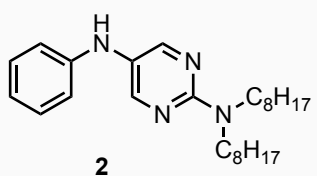

2 e0600 Scan 2 RT=0:29 1008 $=232984 \mathrm{mv} 24-\operatorname{Mar}-2021 \quad 11: 17$ HRP +EI rs-1f 1

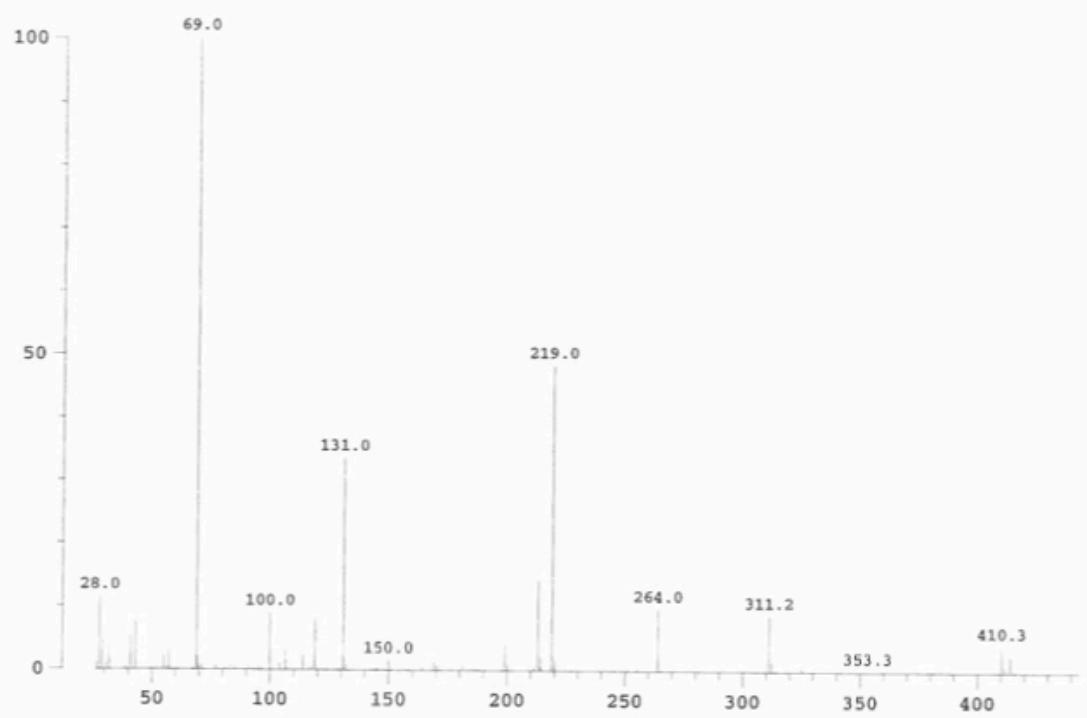

Figure S17. Raw data for EI-HRMS of 2. 


\begin{tabular}{|c|c|c|c|c|c|c|}
\hline \multicolumn{2}{|c|}{ Formula search on } & \multicolumn{2}{|c|}{$\operatorname{scan} 3$} & \multicolumn{3}{|c|}{ of $2 \mathrm{e} 0602$} \\
\hline & & & Dev & $\begin{array}{r}12 \\
\mathrm{C}\end{array}$ & 1 & \\
\hline Mass & Int? & & menus & C & H & N \\
\hline 467.40108 & 7.83 & & 2.29 & 29 & 49 & 5 \\
\hline 466.57817 & 0.01 & No & matehes & found & & \\
\hline 466.51454 & 0.02 & & 4.51 & 30 & 64 & 3 \\
\hline 466.40131 & 25.07 & & -2.24 & 30 & 50 & 4 \\
\hline 466.23639 & 0.04 & & -4.51 & 34 & 30 & 2 \\
\hline 466.03913 & 0.02 & & -1.39 & 35 & 4 & 3 \\
\hline 465.95724 & 0.01 & No & matches & tound & & \\
\hline 465.69502 & 0.01 & No & matches & found & & \\
\hline
\end{tabular}

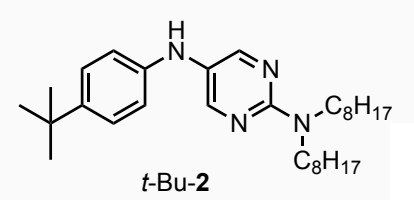

$2 e 0602$ Scan $3 \quad R T=0: 40 \quad 1008=224799 \mathrm{mv} 24-\operatorname{Mar}-2021 \quad 11: 28$
HRP +EI rs $-1 \mathrm{f5}$

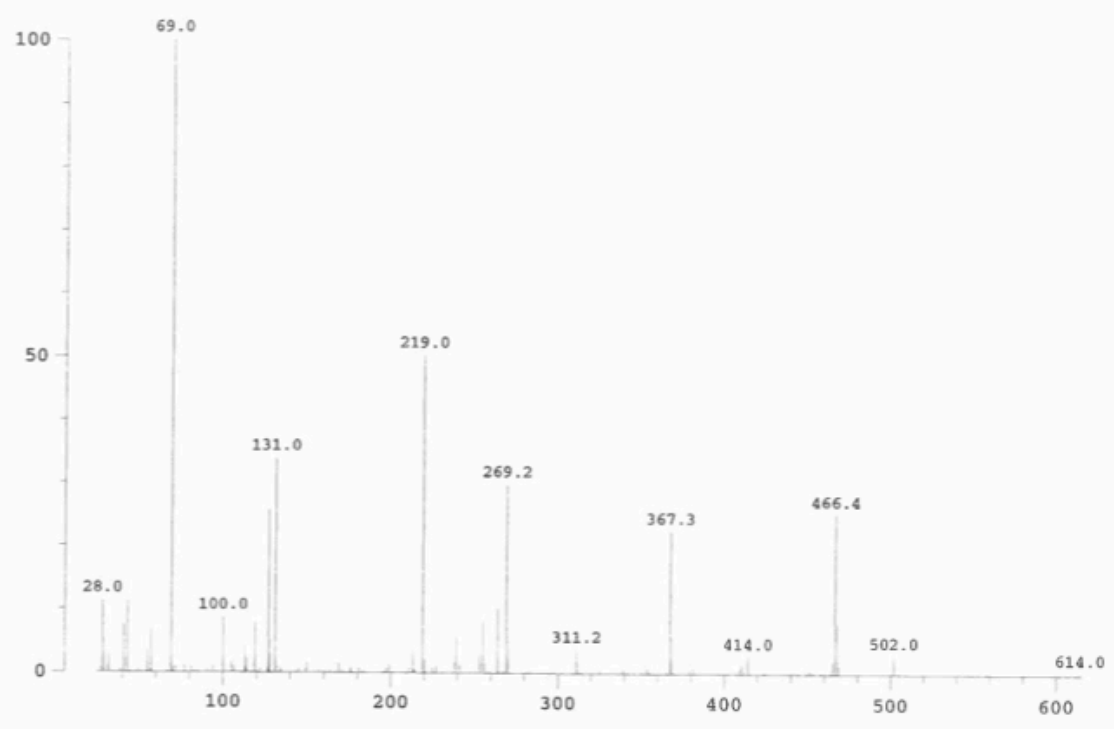

Figure S18. Raw data for EI-HRMS of $t$-Bu-2. 


$\begin{array}{crrrrr}\text { Formula search on scan } 15 & \text { of } 2 \mathrm{e0595} & \\ & & \text { Dev } & 12 & 1 & 14 \\ \text { Mass } & \text { Int? } & \text { menu } & \mathrm{C} & \mathrm{H} & \mathrm{N} \\ 369.29245 & 1.91 & 3.20 & 22 & 35 & 5 \\ 368.29180 & 7.76 & -2.20 & 23 & 36 & 4\end{array}$

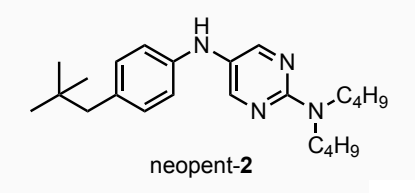

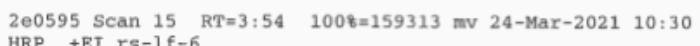

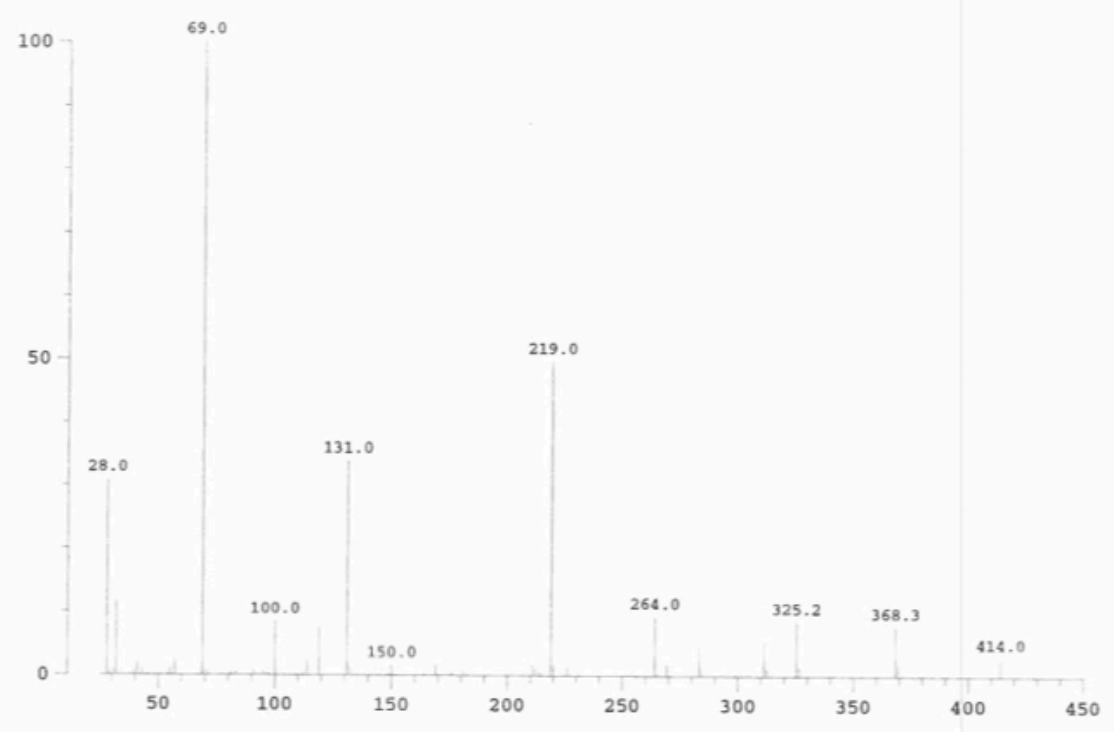

Figure S19. Raw data for EI-HRMS of neopent-2. 


\begin{tabular}{cccccr}
\multicolumn{2}{c}{ Formula search on scan 2} & of 2e0601 \\
& & Dev & 12 & 1 & 14 \\
Mass & Ints & mmu & C & H & N \\
& & & & \\
425.35939 & 1.13 & No matches found & & \\
424.35775 & 4.71 & 1.15 & 27 & 44 & 4
\end{tabular}

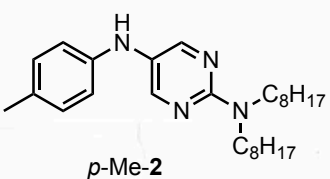

$2 \mathrm{e} 0601$ Scan 2 RT=0:17 $1008=231737$ mv 24-Mar-2021 $11: 23$
HRP +EI rs-1f2

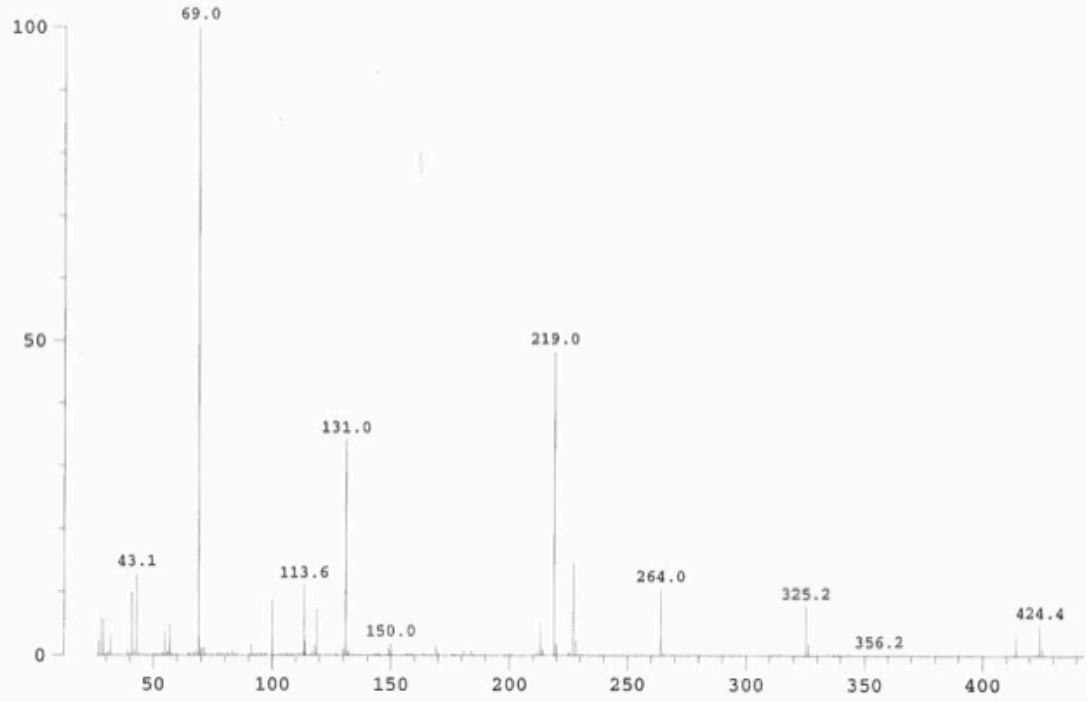

Figure S20. Raw data for EI-HRMS of $p$-Me-2. 


$\begin{array}{crrrrr}\text { Formula search on scan } 2 & \text { of } 2 \mathrm{e} 0599 & \\ & & \text { Dev } & 12 & 1 & 14 \\ \text { Mass } & \text { Int } & \text { menu } & \text { C } & \text { H } & \text { N } \\ 424.35697 & 2.23 & 0.37 & 27 & 44 & 4\end{array}$

,

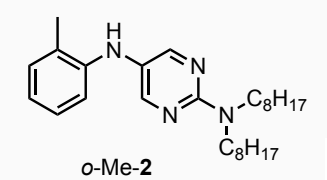

$2 \mathrm{e} 0599 \mathrm{Scan} 2$
HRP +EI rs $-1 \mathrm{fT}=0: 18 \quad 1008=226848 \mathrm{mv} \quad 24-\operatorname{Mar}-2021 \quad 11: 09$

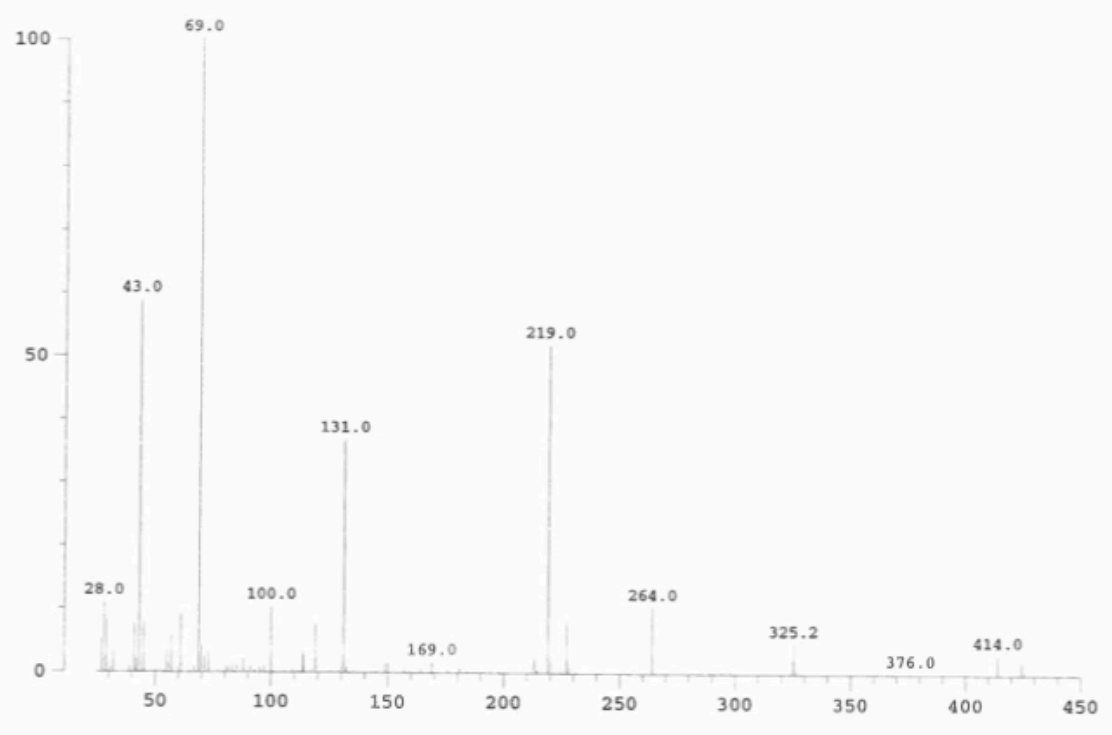

Figure S21. Raw data for EI-HRMS of $o-\mathrm{Me}-2$. 


\begin{tabular}{cccccr} 
Formula search on scan 7 & of 200596 & \\
\multicolumn{1}{c}{ Mass } & Inte & Dev & 12 & 1 & 14 \\
383.31056 & 1.45 & No matches found & H & N \\
382.30735 & 6.59 & -2.30 & 24 & 38 & 4
\end{tabular}

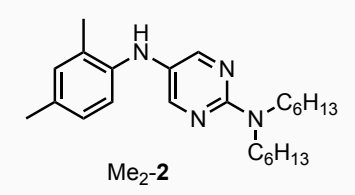

2 e0596 Scan 7 RT=1:44 1008 $=241230 \mathrm{mv} \quad 24-\operatorname{Mar}-2021 \quad 10: 44$ HRP +EI rs-1f-7

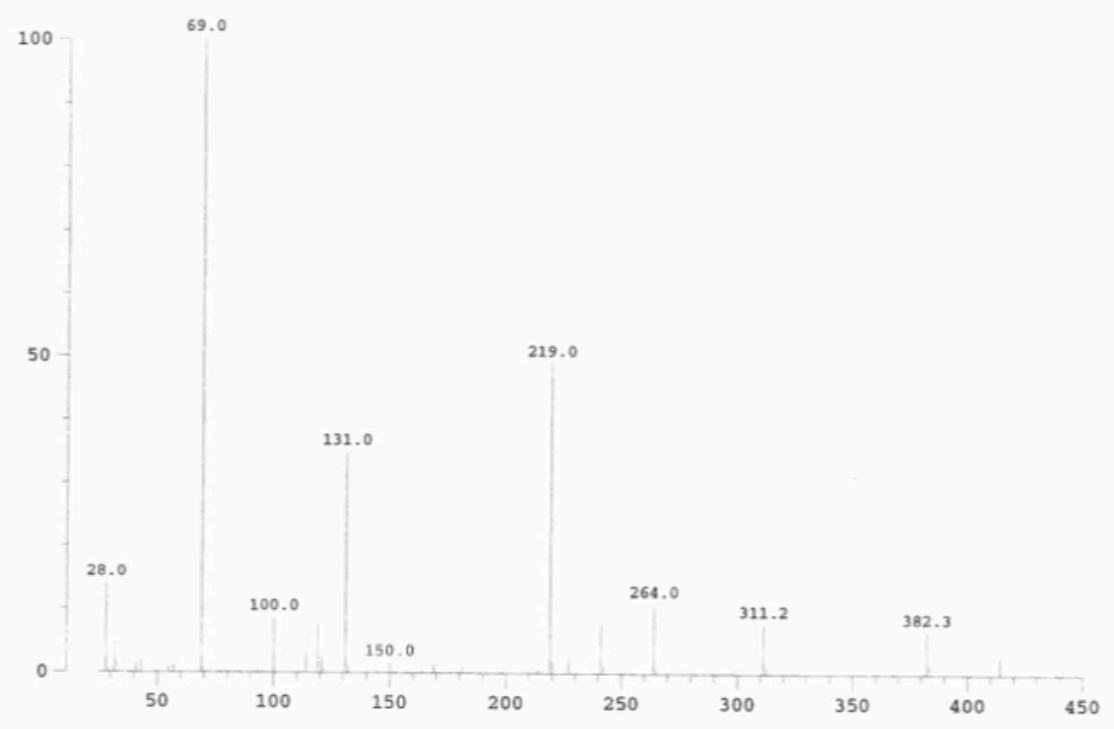

Figure S22. Raw data for EI-HRMS of $\mathrm{Me}_{2}-\mathbf{2}$. 


\begin{tabular}{crrrrr} 
Formula search on scan 7 & \multicolumn{2}{c}{ of 2 e0597 } \\
& & Dev & 12 & 1 & 14 \\
Mass & Int8 & man & C & H & N \\
& & & & & \\
397.32977 & 9.02 & -3.35 & 25 & 41 & 4 \\
396.46578 & 0.02 & -3.73 & 28 & 60 & 0 \\
396.32701 & 36.78 & 1.72 & 25 & 40 & 4 \\
396.08057 & 0.11 & -0.76 & 31 & 10 & 1 \\
395.67236 & 0.01 & No matches found &
\end{tabular}

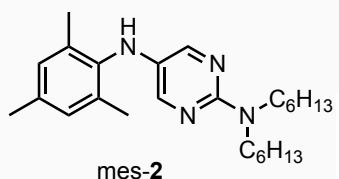
$2 \mathrm{e} 0597 \operatorname{Scan} 7 \quad \mathrm{RT}=1: 51 \quad 1008=219258 \mathrm{mv} 24-\operatorname{Mar}-2021 \quad 10: 53$
HRP +EI rs-1f8

mes-2

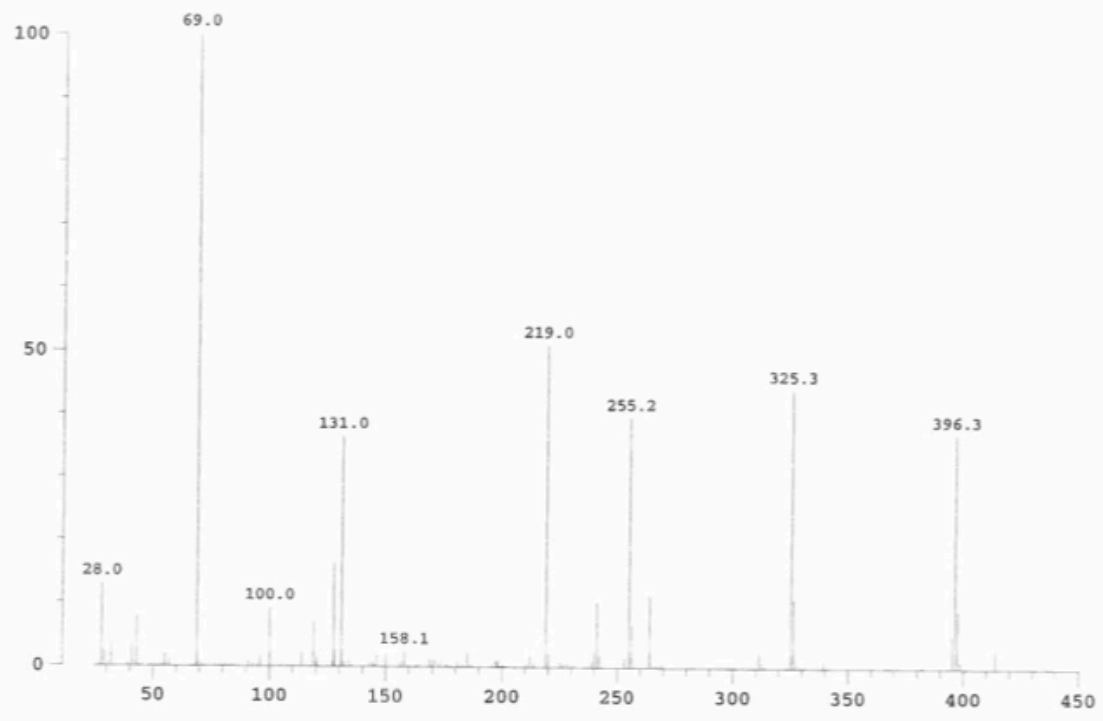

Figure S23. Raw data for EI-HRMS of mes-2. 


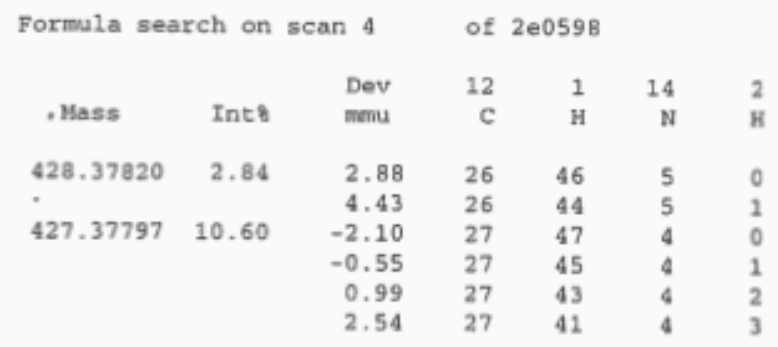

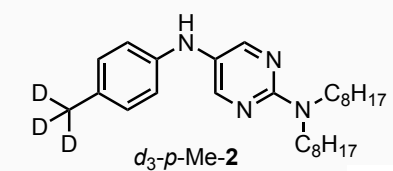

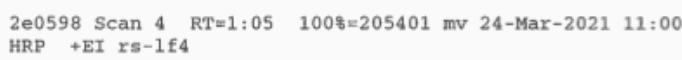

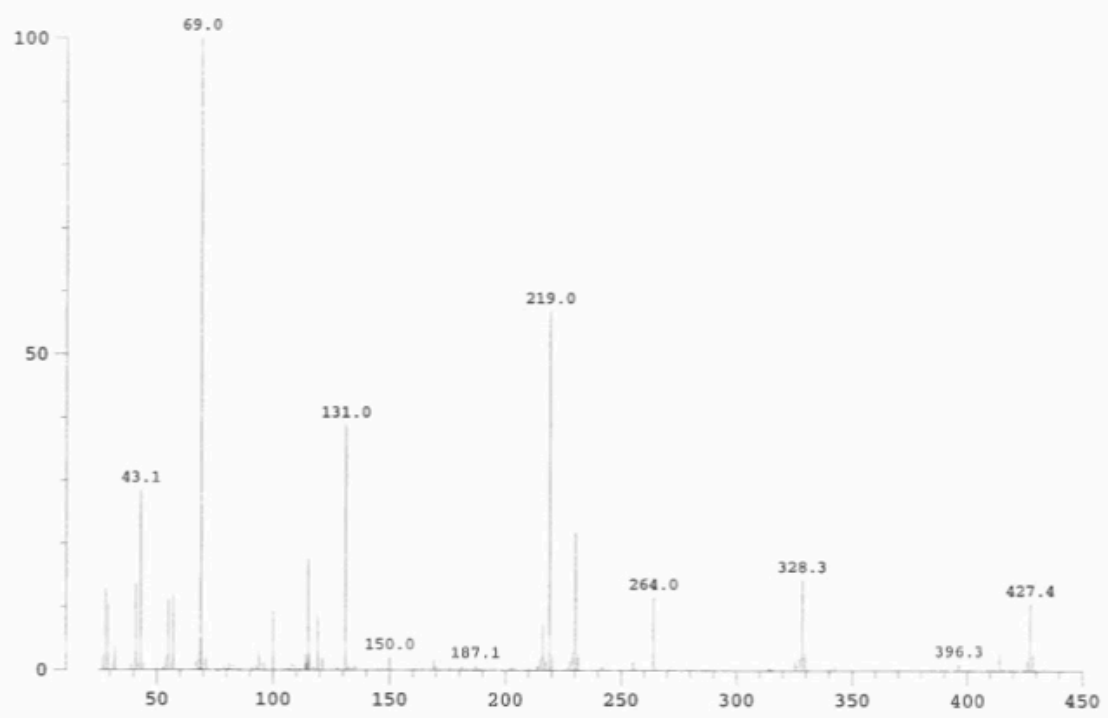

Figure S24. Raw data for EI-HRMS of $d_{3}-p$-Me-2 


\section{Computational Data}

Geometry optimisations of reactants, products and TS structures in gas phase were carried out using the CBS-QB3 complete basis set method as implemented in the Gaussian 16 suite of programs with Opt $=$ Tight and Int $=$ Ultrafine used for convergence criteria (i.e. threshold $=$ 0.000015 and 0.000060 for maximum force and displacement, respectively). In cases where the structures were too large for computations at this level of theory using our computing resources (i.e. N-H BDEs of $t$-Bu-2 and neopent-2), we carried out only the initial B3LYP/CBSB7 steps of the CBS-QB3 calculation. B3LYP or CBS-QB3 enthalpies and free energy (units in Hartrees) are given at $298.15 \mathrm{~K}$ unless otherwise indicated. Frequency calculations confirmed that the optimisation leads to local maxima for TS structures and the corresponding imaginary frequencies (units in $\mathrm{cm}^{-1}$ ) were reported. IRC calculations confirmed that the TS structure connected reported reactants and products. Rate constants were calculated via transition state theory at $37^{\circ} \mathrm{C}$ and 160 ${ }^{\circ} \mathrm{C}$.

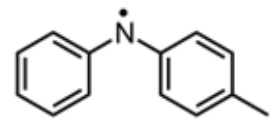

CBS-QB3 Enthalpy $=-556.263165$
CBS-QB3 Free Energy $=-556.316445$

$\begin{array}{lrrr}\text { O } 2 & & & \\ \mathrm{C} & 3.08402100 & 1.43929000 & -0.60996600 \\ \mathrm{C} & 4.20054400 & 0.80704700 & -0.05871500 \\ \mathrm{C} & 4.07032800 & -0.47658600 & 0.48054400 \\ \mathrm{C} & 2.84315000 & -1.11692600 & 0.47087700 \\ \mathrm{C} & 1.68598200 & -0.47434600 & -0.03806700 \\ \mathrm{C} & 1.84195000 & 0.82140100 & -0.59698200 \\ \mathrm{~N} & 0.52737400 & -1.20203900 & -0.03286800 \\ \mathrm{C} & -0.71104800 & -0.63911200 & 0.00017500 \\ \mathrm{C} & -1.79110900 & -1.44638100 & -0.44907100 \\ \mathrm{C} & -3.09044700 & -0.98063600 & -0.43140900 \\ \mathrm{C} & -3.40149500 & 0.29435600 & 0.07300500 \\ \mathrm{C} & -2.34878800 & 1.07828200 & 0.56582900 \\ \mathrm{C} & -1.03497900 & 0.63994900 & 0.52877900 \\ \mathrm{C} & -4.82213900 & 0.79595000 & 0.08137200 \\ \mathrm{H} & 3.18756500 & 2.42027000 & -1.06095600 \\ \mathrm{H} & 5.16564100 & 1.30023800 & -0.06750400 \\ \mathrm{H} & 4.93669900 & -0.97991100 & 0.89514500 \\ \mathrm{H} & 2.72510400 & -2.11924100 & 0.86428600 \\ \mathrm{H} & 0.99303500 & 1.30565300 & -1.06232700 \\ \mathrm{H} & -1.55165600 & -2.43610300 & -0.81868100 \\ \mathrm{H} & -3.89010600 & -1.61567900 & -0.79976600 \\ \mathrm{H} & -2.57009900 & 2.04941200 & 0.99750300 \\ \mathrm{H} & -0.25240100 & 1.25401600 & 0.95534400 \\ \mathrm{H} & -5.51992000 & 0.01320000 & 0.39122600 \\ \mathrm{H} & -5.12853500 & 1.12314600 & -0.91888300 \\ \mathrm{H} & -4.94276200 & 1.64553500 & 0.75645000\end{array}$


<smiles>COOC1(C)C=CC(=Nc2ccccc2)C=C1</smiles>

CBS-QB3 Enthalpy $=-746.272878$

CBS-QB3 Free Energy = -746.334258

CBS-QB3 Enthalpy $(433.15 \mathrm{~K})=-746.256753$

CBS-QB3 Free Energy $(433.15 \mathrm{~K})=-746.364934$

$\begin{array}{lrrr}\mathrm{O}^{1} & & & \\ \mathrm{C} & 4.97266700 & -0.62186000 & 0.16339500 \\ \mathrm{C} & 5.15732600 & 0.72561400 & 0.47528400 \\ \mathrm{C} & 4.10846000 & 1.62149200 & 0.28189500 \\ \mathrm{C} & 2.87848000 & 1.17776500 & -0.19647700 \\ \mathrm{C} & 2.68347400 & -0.17949100 & -0.50107400 \\ \mathrm{C} & 3.75666600 & -1.06963300 & -0.33798600 \\ \mathrm{C} & -0.83102000 & -0.97336400 & -1.28205800 \\ \mathrm{C} & 0.33990800 & -0.52794600 & -0.51732700 \\ \mathrm{~N} & 1.49646300 & -0.66040500 & -1.07064200 \\ \mathrm{C} & -2.07482000 & -0.88787200 & -0.80175800 \\ \mathrm{C} & -2.39288600 & -0.38621900 & 0.58268900 \\ \mathrm{C} & -1.16864400 & 0.09752400 & 1.31551900 \\ \mathrm{C} & 0.07188800 & 0.01768000 & 0.82371200 \\ \mathrm{C} & -3.10700600 & -1.47054000 & 1.41040500 \\ \mathrm{O} & -3.21768200 & 0.81018100 & 0.52337900 \\ \mathrm{O} & -4.49736300 & 0.45513700 & -0.09264500 \\ \mathrm{C} & -4.76034700 & 1.48184800 & -1.03361100 \\ \mathrm{H} & 5.78605900 & -1.32618200 & 0.29924500 \\ \mathrm{H} & 6.11230800 & 1.07428900 & 0.85076500 \\ \mathrm{H} & 4.24570600 & 2.67444000 & 0.50283200 \\ \mathrm{H} & 2.07165700 & 1.88128600 & -0.36711100 \\ \mathrm{H} & 3.61225600 & -2.10937600 & -0.60655000 \\ \mathrm{H} & -0.62494600 & -1.35742000 & -2.27460900 \\ \mathrm{H} & -2.92514800 & -1.20077700 & -1.39719400 \\ \mathrm{H} & -1.34751100 & 0.49960600 & 2.30762300 \\ \mathrm{H} & 0.91628500 & 0.33827600 & 1.42063300 \\ \mathrm{H} & -2.46467500 & -2.34669200 & 1.52012900 \\ \mathrm{H} & -3.34973500 & -1.07888600 & 2.40093300 \\ \mathrm{H} & -4.03339100 & -1.76786700 & 0.91828900 \\ \mathrm{H} & -5.74231300 & 1.23256100 & -1.44324900 \\ \mathrm{H} & -4.80420500 & 2.46058700 & -0.54636400 \\ \mathrm{H} & -4.01210600 & 1.49645100 & -1.83240400 \\ & & & \end{array}$<smiles>COc1ccc(/C=N/c2ccccc2)cc1</smiles>

CBS-QB3 Enthalpy = -746.213685

CBS-QB3 Free Energy = -746.276149

CBS-QB3 Enthalpy $(433.15 \mathrm{~K})=-746.19775$

CBS-QB3 Free Energy $(433.15 \mathrm{~K})=-746.307282$

Imaginary Frequency $=-210.90$ 


3.68773500
4.96632700
5.13145600
4.03343900
2.72894600
2.57656500
1.68885400
0.50524100
-0.53026600
-1.80036700
-2.15539400
-1.13326700
0.13654100
-3.49491500
3.55508300
5.82814400
6.12422800
4.14638100
1.59186700
-0.24411200
-2.56661100
-1.39035700
0.89869500
-4.06269000
-3.54753600
-4.02123100
-3.61369400
-4.18345800
-4.79964200
-5.20328900
-4.06122400
-5.60650300
-1

1.86126100

1.31340100

0.04541100

$-0.66576800$

$-0.14807700$

1.14263200

$-0.89960500$

$-1.00195900$

$-1.63617700$

$-1.76945600$

$-1.35322700$

$-0.75846300$

$-0.59013600$

$-1.54762100$

2.85917500

1.87706500

$-0.37996600$

$-1.63901100$

1.59190900

$-1.96785100$

$-2.21815800$

$-0.46052700$

$-0.18235900$

$-2.32966200$

$-1.63690100$

$-0.52864200$

0.80119800

2.04113400

2.12467800

3.13646900

1.94936200

1. 38867600
0.54890500

0.45130500

$-0.11096700$

$-0.57014700$

$-0.42790000$

0.12483500

$-0.94630700$

$-0.38875600$

$-1.18013100$

$-0.71599700$

0.60482400

1.41054200

0.95346400

1.12178000

0.95200100

0.78906100

$-0.20629100$

$-1.03207500$

0.16550500

$-2.17072100$

$-1.33811300$

2. 42075400

1.60369800

0.61730500

2.20735500

0.87249600

0.23755200

0.08154700

$-1.19950700$

$-1.27383000$

$-1.98753300$

$-1.28175300$<smiles>C=C1C=CC(=Nc2ccccc2)C=C1</smiles>

\section{CBS-QB3 Enthalpy $=-555.665403$ \\ CBS-QB3 Free Energy = $\mathbf{- 5 5 5 . 7 1 5 4 8 4}$}

${ }^{0}{ }^{1}$
$\mathrm{C}^{2}$
$\mathrm{C}$
$\mathrm{C}$
$\mathrm{C}$
$\mathrm{C}$
$\mathrm{C}$
$\mathrm{N}$
$\mathrm{C}$
$\mathrm{C}$
$\mathrm{C}$
$\mathrm{C}$
$\mathrm{C}$

-3.15768000
-4.12979000
-3.84514800
-2.60671900
-1.60644600
-1.90477400
-0.40001400
0.76139300
1.93668200
3.18847800
3.44843000
2.28150300

1.28214400

0.72812300

$-0.44674200$

$-1.06196700$

$-0.49001700$

0.68717900

$-1.18053300$

$-0.63031900$

$-1.44313800$

$-0.96388900$

0.38994500

1. 18560800
$-0.73546100$

0.09452800

0.79187500

0.66077300

$-0.14345200$

$-0.85244500$

$-0.29190300$

$-0.12965100$

$-0.44490600$

$-0.33080900$

0.14550100

0.50949500 


$\begin{array}{lrrr}\mathrm{C} & 1.02437600 & 0.71992700 & 0.37935900 \\ \mathrm{C} & 4.70209300 & 0.87889200 & 0.26235800 \\ \mathrm{H} & -3.37463700 & 2.18291700 & -1.29943400 \\ \mathrm{H} & -5.10299100 & 1.19634600 & 0.18548200 \\ \mathrm{H} & -4.59868100 & -0.89365200 & 1.43115500 \\ \mathrm{H} & -2.38478900 & -1.98769400 & 1.17822800 \\ \mathrm{H} & -1.16092500 & 1.10792800 & -1.51916500 \\ \mathrm{H} & 1.73695100 & -2.44898700 & -0.79434200 \\ \mathrm{H} & 4.04114400 & -1.58280300 & -0.59023000 \\ \mathrm{H} & 2.45603400 & 2.18000600 & 0.90844500 \\ \mathrm{H} & 0.18072800 & 1.32871600 & 0.67729000 \\ \mathrm{H} & 5.56778100 & 0.28231300 & -0.00049200 \\ \mathrm{H} & 4.88509500 & 1.88415900 & 0.62339100\end{array}$<smiles>Cc1ccc(Nc2cnc(N(C)C)nc2)cc1</smiles>

CBS-QB3 Enthalpy $=-722.078190$

CBS-QB3 Free Energy = -722.142223

DFT Enthalpy $=-723.296968$

DFT Free Energy $=-722.078190$

1.17164000

0.54984100

$-0.73005700$

$-1.35391600$

$-0.71007700$

0.57168100

$-1.42507900$

$-0.85035600$

$-1.66663800$

$-1.24443300$

0.04689500

0.89771200

0.46031700

0.51102700

$-0.33085000$

1.87096600

1.22556000

2.14408900

$-1.24569600$

$-2.34967100$

1.06566600

$-2.70320200$

1.14459700

0.12842800

$-1.30788900$

$-0.44425300$

2.40085300

2.39363400

1.85828300
0.62285200

0.07943700

$-0.47544700$

$-0.49417600$

0.00678700

0.58609300

$-0.03224000$

$-0.03907400$

0.29116100

0.30402900

$-0.05220800$

$-0.46781800$

$-0.45378600$

$-0.00779600$

0.40657900

$-0.39368900$

0.08701900

1.09398400

$-0.88464500$

$-0.90384200$

1.05822000

0.56104900

$-0.83485300$

1.25345000

0.69535000

$-0.41539700$

0.45097300

$-0.70737500$

$-1.21774100$ 


$\begin{array}{lrrr}\mathrm{H} & 6.67799000 & 0.52470300 & 0.35102900 \\ \mathrm{H} & 6.12399300 & 1.63242300 & -0.90135100 \\ \mathrm{H} & 5.90915300 & 2.05339300 & 0.79862300\end{array}$<smiles>COC1(C)C=CC(=Nc2cnc(N(C)C)nc2)C=C1</smiles>

\section{CBS-QB3 Enthalpy $=-912.084653$ \\ CBS-QB3 Free Energy = -912.156934 \\ CBS-QB3 Enthalpy $(433.15 \mathrm{~K})=-912.065276$ \\ CBS-QB3 Free Energy $(433.15 \mathrm{~K})=-912.193138$}

\begin{tabular}{lrrr}
$\mathrm{O}_{1}$ & & \\
$\mathrm{C}$ & 3.83633100 & 0.11553300 & -0.03761100 \\
$\mathrm{C}$ & 2.43945800 & -1.58562600 & -0.62652300 \\
$\mathrm{C}$ & 1.31600400 & -0.74204000 & -0.57082600 \\
$\mathrm{C}$ & 1.62140000 & 0.60236300 & -0.30061100 \\
$\mathrm{~N}$ & 0.07308600 & -1.25245100 & -0.93013600 \\
$\mathrm{C}$ & -1.03873200 & -0.93046800 & -0.34991500 \\
$\mathrm{C}$ & -2.27044100 & -1.44769900 & -0.95353600 \\
$\mathrm{C}$ & -3.48303400 & -1.14744000 & -0.47781600 \\
$\mathrm{C}$ & -3.70190900 & -0.30486400 & 0.74896500 \\
$\mathrm{C}$ & -2.40953400 & 0.16124900 & 1.36482200 \\
$\mathrm{C}$ & -1.20098200 & -0.12357000 & 0.86720200 \\
$\mathrm{C}$ & -4.54678500 & -1.06498700 & 1.78774500 \\
$\mathrm{H}$ & 2.30195900 & -2.62843700 & -0.90114800 \\
$\mathrm{H}$ & 0.84457200 & 1.36150300 & -0.34453800 \\
$\mathrm{H}$ & -2.13696500 & -2.06599600 & -1.83391800 \\
$\mathrm{H}$ & -4.38005300 & -1.51817800 & -0.96476400 \\
$\mathrm{H}$ & -2.50961000 & 0.75192500 & 2.27045600 \\
$\mathrm{H}$ & -0.31178800 & 0.21748500 & 1.38213500 \\
$\mathrm{H}$ & -5.50238600 & -1.35931500 & 1.34865100 \\
$\mathrm{H}$ & -4.01518400 & -1.95959300 & 2.11544300 \\
$\mathrm{H}$ & -4.74122000 & -0.42596600 & 2.65153000 \\
$\mathrm{O}$ & -4.55528400 & 0.83313800 & 0.45443300 \\
$\mathrm{O}$ & -3.84802100 & 1.71032500 & -0.47305600 \\
$\mathrm{C}$ & -4.73868300 & 1.94784400 & -1.54822700 \\
$\mathrm{H}$ & -4.20879300 & 2.65789200 & -2.18784000 \\
$\mathrm{H}$ & -4.95036200 & 1.03182600 & -2.10930200 \\
$\mathrm{H}$ & -5.67311000 & 2.39743100 & -1.19762400 \\
$\mathrm{~N}$ & 2.84867300 & 1.03442300 & -0.04472800 \\
$\mathrm{~N}$ & 3.67042400 & -1.18948100 & -0.35188600 \\
$\mathrm{~N}$ & 5.09475300 & 0.53304000 & 0.29469000 \\
$\mathrm{C}$ & 6.23410800 & -0.36789300 & 0.26401900 \\
$\mathrm{H}$ & 5.88791500 & -1.38590200 & 0.11524100 \\
$\mathrm{H}$ & 6.91961900 & -0.10050600 & -0.55064900 \\
$\mathrm{H}$ & 6.78600800 & -0.30151300 & 1.20805600 \\
$\mathrm{C}$ & 5.39099700 & 1.92792000 & 0.57208700 \\
$\mathrm{H}$ & 4.46731300 & 2.49772700 & 0.59226000 \\
$\mathrm{H}$ & 5.89775800 & 2.01823200 & 1.53946500 \\
$\mathrm{H}$ & 6.05302800 & 2.34302400 & -0.19870400 \\
& & & \\
\hline & & & \\
& & &
\end{tabular}




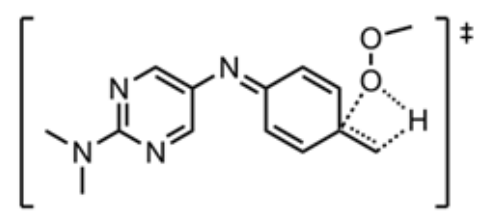

CBS-QB3 Enthalpy $=-912.043801$
CBS-QB3 Free Energy $=-912.117245$
CBS-QB3 Enthalpy $(433.15 \mathrm{~K})=-912.024619$
CBS-QB3 Free Energy $(433.15 \mathrm{~K})=-912.153942$
Imaginary Frequency $=-129.18$

\begin{tabular}{lrrr}
$\mathrm{O}^{1}$ & & & \\
$\mathrm{C}$ & 3.95489900 & 0.31974100 & -0.07682300 \\
$\mathrm{C}$ & 2.79208000 & -1.49434300 & -0.80867800 \\
$\mathrm{C}$ & 1.55064800 & -0.88419500 & -0.49650900 \\
$\mathrm{C}$ & 1.67456200 & 0.45395700 & -0.04452800 \\
$\mathrm{~N}$ & 0.41343200 & -1.57561000 & -0.78858900 \\
$\mathrm{C}$ & -0.76248100 & -1.38834700 & -0.20538200 \\
$\mathrm{C}$ & -1.89269800 & -2.02081100 & -0.83687500 \\
$\mathrm{C}$ & -3.15929300 & -1.86875600 & -0.35424800 \\
$\mathrm{C}$ & -3.41282500 & -1.13557100 & 0.83911300 \\
$\mathrm{C}$ & -2.29541900 & -0.54882000 & 1.50150000 \\
$\mathrm{C}$ & -1.02549400 & -0.66438600 & 1.01490300 \\
$\mathrm{C}$ & -4.76109300 & -0.98994900 & 1.37077200 \\
$\mathrm{H}$ & 2.78196200 & -2.49338300 & -1.23673800 \\
$\mathrm{H}$ & 0.79303200 & 1.07408700 & 0.08805700 \\
$\mathrm{H}$ & -1.68885300 & -2.59294800 & -1.73379200 \\
$\mathrm{H}$ & -3.99672400 & -2.32553200 & -0.86985000 \\
$\mathrm{H}$ & -2.47219800 & -0.01431100 & 2.42797400 \\
$\mathrm{H}$ & -0.19844200 & -0.25360500 & 1.57866900 \\
$\mathrm{H}$ & -5.45422600 & -1.76138700 & 1.03309200 \\
$\mathrm{H}$ & -4.79475000 & -0.86886000 & 2.45467900 \\
$\mathrm{H}$ & -5.12770300 & 0.02221800 & 0.93934300 \\
$\mathrm{O}$ & -4.57766700 & 1.22510300 & 0.08535800 \\
$\mathrm{O}$ & -5.03204300 & 2.48533600 & -0.21775300 \\
$\mathrm{C}$ & -5.68372200 & 2.46318900 & -1.48219800 \\
$\mathrm{H}$ & -5.99100100 & 3.49181600 & -1.68308600 \\
$\mathrm{H}$ & -4.99535200 & 2.11154600 & -2.25689400 \\
$\mathrm{H}$ & -6.5599400 & 1.80657000 & -1.44691600 \\
$\mathrm{~N}$ & 2.83775300 & 1.04637700 & 0.15367400 \\
$\mathrm{~N}$ & 3.96331600 & -0.93484600 & -0.59226500 \\
$\mathrm{~N}$ & 5.15507300 & 0.89710600 & 0.19885700 \\
$\mathrm{C}$ & 6.41465000 & 0.20763400 & -0.03546100 \\
$\mathrm{H}$ & 6.21750700 & -0.79855200 & -0.39071900 \\
$\mathrm{H}$ & 7.00735600 & 0.74712400 & -0.78322800 \\
$\mathrm{H}$ & 6.99393700 & 0.16412900 & 0.89333100 \\
$\mathrm{C}$ & 5.25711600 & 2.24956200 & 0.72528300 \\
$\mathrm{H}$ & 4.26496800 & 2.67931500 & 0.81777400 \\
$\mathrm{H}$ & 5.74264900 & 2.23653600 & 1.70769300 \\
$\mathrm{H}$ & 5.86287400 & 2.86710300 & 0.05281300 \\
& & & \\
\hline & & & \\
& & &
\end{tabular}


<smiles>C=C1C=CC(=Nc2cnc(N(C)C)nc2)C=C1</smiles>

CBS-QB3 Enthalpy $=-721.475225$

CBS-QB3 Free Energy = -721.536234

$$
\begin{array}{r}
2.64048000 \\
2.92744800 \\
2.03531400 \\
0.77732100 \\
0.34416400 \\
1.38304400 \\
-0.92938600 \\
-2.02650800 \\
-3.28025200 \\
-4.47623100 \\
-4.59312100 \\
-3.34923700 \\
-2.14744400 \\
-5.78814700 \\
4.21367400 \\
5.25813700 \\
4.63948600 \\
0.08237700 \\
1.14840800 \\
-3.18859700 \\
-5.38848800 \\
-3.41660900 \\
-1.25125500 \\
-6.71227100 \\
-5.86257100 \\
4.81689500 \\
5.99094500 \\
5.78517500 \\
5.11933200 \\
5.36622300 \\
3.77790100
\end{array}
$$

0.40811600

$-0.03787500$

$-0.62200800$

$-0.68477100$

$-0.18986100$

0.31001400

$-0.29059100$

$-0.10506200$

$-0.40363200$

$-0.29334000$

0.17551600

0.54674700

0.41294500

0.28791500

0.09825200

0.65705600

$-0.41460600$

$-1.19890400$

0.64706100

$-0.74845500$

$-0.54973100$

0.95986000

0.73244800

0.02436700

0.64602800

1.06189500

$-0.11349400$

1. 45181700

0.38138000

$-1.22682600$

$-0.79011100$

\section{$\mathrm{MeOOH}$}

\section{CBS-QB3 Enthalpy $=-190.589542$}

\section{CBS-QB3 Free Energy $=-190.620447$}

\author{
01

$\mathrm{C}$
$\mathrm{O}$
$\mathrm{O}$
$\mathrm{H}$
$\mathrm{H}$
$\mathrm{H}$
$\mathrm{H}$

\section{H-atom}

-0.22363900
0.60684200
-0.28550800
0.47126800
-0.87767400
-0.82582100
0.00339000

0.02672600

$-0.03138300$

$-0.09072600$

0.02466200

$-0.84877700$

0.94203100

0.69859800 
CBS-QB3 Enthalpy $=-0.497457$

CBS-QB3 Free Energy $=-0.510472$

DFT Enthalpy $=-0.499795$

DFT Free Energy $=-0.512810$

02

$\mathrm{H}$<smiles>Cc1ccc(N([O])c2ccccc2)cc1</smiles>

CBS-QB3 Enthalpy $=-631.374519$

CBS-QB3 Free Energy = -631.429937

02

C

C

C

C

C

C

$\mathrm{N}$

C

C

C

C

C

C

C

O

$\mathrm{H}$

$\mathrm{H}$

$\mathrm{H}$

$\mathrm{H}$

$\mathrm{H}$

$\mathrm{H}$

$\mathrm{H}$

$\mathrm{H}$

$\mathrm{H}$

$\mathrm{H}$

$\mathrm{H}$

0.00000000
0.00000000

0.00000000

$\begin{array}{rrr}4.05418100 & 0.12328200 & -0.48323800 \\ 4.10345900 & -1.13806100 & 0.11117500 \\ 2.94849400 & -1.67713600 & 0.67461400 \\ 1.74816000 & -0.97440900 & 0.63802800 \\ 1.70234700 & 0.28330300 & 0.02442500 \\ 2.86413700 & 0.83745800 & -0.52591800 \\ 0.51075100 & 1.06707600 & -0.00369100 \\ -0.80442900 & 0.51375300 & -0.01522000 \\ -1.84432800 & 1.27479800 & 0.53003500 \\ -3.14383900 & 0.79169300 & 0.49670500 \\ -3.45023300 & -0.44802500 & -0.07970900 \\ -2.40086600 & -1.18329200 & -0.63860100 \\ -1.09055700 & -0.71682300 & -0.61442900 \\ -4.86404400 & -0.97425900 & -0.08315200 \\ 0.62827200 & 2.34246000 & -0.01907900 \\ 4.94912200 & 0.55423900 & -0.91746200 \\ 5.03485400 & -1.69113000 & 0.14259600 \\ 2.98088300 & -2.64652300 & 1.15880000 \\ 0.86328500 & -1.38696100 & 1.10378400 \\ 2.80832000 & 1.82334600 & -0.96609400 \\ -1.61104400 & 2.24035800 & 0.95725500 \\ -3.93953900 & 1.39121700 & 0.92706400 \\ -2.61050400 & -2.13432000 & -1.11706700 \\ -0.30409600 & -1.29305600 & -1.08352400 \\ -5.00347200 & -1.73779300 & -0.85134100 \\ -5.11781700 & -1.42775400 & 0.88144100 \\ -5.58631600 & -0.17453000 & -0.26526800\end{array}$<smiles>Cc1ccc(N(O)c2ccccc2)cc1</smiles>

CBS-QB3 Enthalpy $=-631.985255$

CBS-QB3 Free Energy $=-632.041608$ 


$$
\begin{array}{r}
3.73156800 \\
4.03368300 \\
3.15459000 \\
1.98929100 \\
1.67711600 \\
2.55721100 \\
0.48666500 \\
-0.79923000 \\
-1.93475000 \\
-3.20456500 \\
-3.40002400 \\
-2.26213900 \\
-0.97769500 \\
-4.78912900 \\
4.40988400 \\
4.94788100 \\
3.38524700 \\
1.31822100 \\
2.31938700 \\
-1.80916400 \\
-4.06580200 \\
-2.37249300 \\
-0.12090600 \\
-4.76579100 \\
-5.27460900 \\
-5.42943600 \\
0.56654800 \\
0.86297000
\end{array}
$$

$$
\begin{array}{r}
-1.02044400 \\
-0.18147900 \\
0.84997000 \\
1.04387600 \\
0.18497100 \\
-0.84816000 \\
0.43131100 \\
0.19013900 \\
0.40736700 \\
0.22498000 \\
-0.16461100 \\
-0.38320700 \\
-0.22147000 \\
-0.32884300 \\
-1.82287200 \\
-0.32197000 \\
1.51965100 \\
1.86123800 \\
-1.49414200 \\
0.69788600 \\
0.39296100 \\
-0.70410500 \\
-0.42270600 \\
-0.84081300 \\
0.64124800 \\
-0.90789300 \\
-0.09914100 \\
0.65693300
\end{array}
$$<smiles>C=C1C=CC(=[N+]([O-])c2ccccc2)C=C1</smiles>

\section{CBS-QB3 Enthalpy $=-630.760033$ CBS-QB3 Free Energy $=-630.811892$}

$$
\begin{array}{ll}
O^{C}{ }^{C} \\
C \\
C \\
C \\
C \\
C \\
C \\
\text { N } \\
C \\
C \\
C \\
C \\
C \\
C \\
C \\
C \\
O \\
O \\
H
\end{array}
$$

$$
\begin{array}{rr}
0.08699700 & -0.85417900 \\
-1.03458500 & -0.05468700 \\
-1.47506200 & 0.81859300 \\
-0.81069800 & 0.88526500 \\
0.29576900 & 0.06771500 \\
0.76460900 & -0.78644000 \\
1.06620500 & 0.14986100 \\
0.50111100 & 0.05741500 \\
1.33125000 & 0.30203300 \\
0.82030200 & 0.21812300 \\
-0.56852100 & -0.14073200 \\
-1.37149200 & -0.42055600 \\
-0.87226400 & -0.32473900 \\
-1.07821100 & -0.21957100 \\
2.32292500 & 0.30538800 \\
0.43924200 & -1.52620900
\end{array}
$$




$\begin{array}{rrrr}\mathrm{H} & -4.98985000 & -1.55475800 & -0.10391900 \\ \mathrm{H} & -3.22791600 & -2.33043700 & 1.45964100 \\ \mathrm{H} & -1.05980800 & -1.13258800 & 1.57930000 \\ \mathrm{H} & -2.41724200 & 1.65613800 & -1.36990900 \\ \mathrm{H} & 1.80860600 & 2.36374300 & 0.56375100 \\ \mathrm{H} & 4.09634800 & 1.45245500 & 0.42396800 \\ \mathrm{H} & 2.45106000 & -2.40005400 & -0.73211700 \\ \mathrm{H} & 0.20343000 & -1.49603700 & -0.57135600 \\ \mathrm{H} & 5.60972300 & -0.46633500 & -0.01644200 \\ \mathrm{H} & 4.91221000 & -2.11343300 & -0.48827800\end{array}$<smiles>Cc1ccc(Nc2ccccc2)cc1</smiles>

\section{CBS-QB3 Enthalpy $=-556.897215$ CBS-QB3 Free Energy = -556.950864}

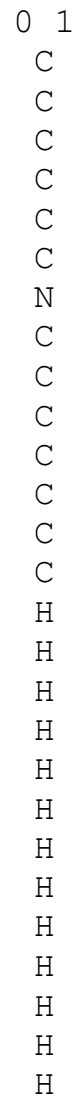

$$
\begin{array}{rr}
2.43475600 & -1.08847400 \\
3.47302500 & -0.28856400 \\
3.13160500 & 0.97727200 \\
1.81337700 & 1.41442200 \\
0.77682200 & 0.60291500 \\
1.11173500 & -0.65906500 \\
-0.52853300 & 1.11011400 \\
-1.74839700 & 0.43099000 \\
-2.90216200 & 1.09989300 \\
-4.14728300 & 0.48760300 \\
-4.27563400 & -0.81136400 \\
-3.13570700 & -1.47844600 \\
-1.88457400 & -0.87075300 \\
4.90035400 & -0.77984800 \\
2.66397500 & -2.06893500 \\
3.90980600 & 1.63319600 \\
1.57716700 & 2.39537700 \\
0.34333900 & -1.29253700 \\
-0.59807500 & 2.11503500 \\
-2.81086800 & 2.10458500 \\
-5.02158400 & 1.02730200 \\
-5.24568000 & -1.29217800 \\
-3.21757200 & -2.48382100 \\
-1.01914400 & -1.39469700 \\
5.13382100 & -1.28975500 \\
5.08882100 & -1.48975100
\end{array}
$$

$-0.54079300$

$-0.05530600$

0.43076400

0.45506700

$-0.03022300$

$-0.54165300$

$-0.03856200$

0.00246100

$-0.44150500$

$-0.37966400$

0.11004200

0.55281800

0.51066500

$-0.03468000$

$-0.94699000$

0.80850500

0.85590000

$-0.96469200$

$-0.05697500$

$-0.84271900$

$-0.72681800$

0.14880400

0.95158800

0.89342800

0.90697000

$-0.84385600$

$-0.14115600$<smiles>CNc1ncc(N([O-])c2ccc(C)cc2)cn1</smiles> 


\section{CBS-QB3 Enthalpy $=-797.188000$ \\ CBS-QB3 Free Energy = -797.254622}

O 2
N
C
N
C
C
C
N
C
C
C
C
C
C
N
C
C
C
O
H
H
H
H
H
H
H
H
H
H
H
H
H
H
H

$$
\begin{array}{rr}
-2.08391800 & -0.94588400 \\
-3.03332700 & -0.17539500 \\
-2.81071300 & 1.05023000 \\
-1.56867900 & 1.49972300 \\
-0.50513200 & 0.75989800 \\
-0.84608300 & -0.47427900 \\
0.79338700 & 1.32912700 \\
1.99043700 & 0.56512800 \\
3.16600000 & 1.12617700 \\
4.35883700 & 0.42498500 \\
4.42599900 & -0.84213700 \\
3.24617500 & -1.37658500 \\
2.03854900 & -0.69030300 \\
-4.30556800 & -0.66364400 \\
-4.65715900 & -1.95105800 \\
-5.40538800 & 0.10661000 \\
5.72612200 & -1.60503500 \\
0.87997400 & 2.60617900 \\
-1.38117600 & 2.48958800 \\
-0.09830300 & -1.09340900 \\
3.11678200 & 2.11049300 \\
5.26078400 & 0.87159200 \\
3.27013500 & -2.34404400 \\
1.15133100 & -1.11662100 \\
-5.15394700 & -2.57402000 \\
-5.34704000 & -1.81859000 \\
-3.75926500 & -2.45066700 \\
-5.91012900 & -0.47350700 \\
-6.14071700 & 0.34302400 \\
-5.02410300 & 1.03015000 \\
5.69518900 & -2.38936000 \\
6.56596400 & -0.94433200 \\
5.94427200 & -2.08490900
\end{array}
$$$$
-0.60801700
$$$$
-0.03188700
$$$$
0.49777800
$$$$
0.47445300
$$$$
-0.05940400
$$$$
-0.61961900
$$$$
-0.09963800
$$$$
-0.02255600
$$$$
-0.53738500
$$$$
-0.45343300
$$$$
0.14182400
$$$$
0.66737100
$$$$
0.59381700
$$$$
0.01491400
$$$$
-0.56142400
$$$$
0.57290100
$$$$
0.19890200
$$$$
-0.19901500
$$$$
0.87631200
$$

$-1.10585600$

$-0.98179000$

$-0.85957100$

1.15801300

1.04304500

0.19066800

$-1.40389500$

$-0.91089000$

1.35342100

$-0.20587300$

0.99662600

0.95808100

0.42883800

$-0.76163100$<smiles>CNc1ncc(N(O)c2ccc(C)cc2)cn1</smiles>

\section{CBS-QB3 Enthalpy = -797.793313}

CBS-QB3 Free Energy $=-797.860725$

$$
\begin{gathered}
0^{0} 1 \\
N^{N} \\
\text { C } \\
\text { N } \\
\text { C } \\
\text { C } \\
\text { C }
\end{gathered}
$$

2.74519400

2.98911000

2.07054400

0.84894300

0.48888200

1.51214300
0.79449800
-0.18146500
-0.69058500
-0.18048800
0.82364400
1.27315200

$$
\begin{array}{r}
-0.89244300 \\
0.01387000 \\
0.86752800 \\
0.80332900 \\
-0.09857400 \\
-0.93585600
\end{array}
$$




$\begin{array}{lrrr}\mathrm{N} & -0.80096100 & 1.42188300 & -0.15881800 \\ \mathrm{C} & -1.96916600 & 0.62664000 & -0.10688400 \\ \mathrm{C} & -3.13972400 & 1.08943700 & 0.50291800 \\ \mathrm{C} & -4.29423900 & 0.30813300 & 0.48638800 \\ \mathrm{C} & -4.33236300 & -0.94030200 & -0.13254500 \\ \mathrm{C} & -3.15766900 & -1.38185600 & -0.75527900 \\ \mathrm{C} & -1.99704900 & -0.62218300 & -0.75111500 \\ \mathrm{~N} & 4.25192200 & -0.69109100 & 0.06763600 \\ \mathrm{C} & 5.31624000 & -0.20657700 & -0.79663100 \\ \mathrm{C} & 4.62218000 & -1.73673900 & 1.00753100 \\ \mathrm{C} & -5.58113900 & -1.78873800 & -0.13447900 \\ \mathrm{O} & -0.86605300 & 2.59900200 & 0.63835900 \\ \mathrm{H} & 0.11316500 & -0.60000100 & 1.48615800 \\ \mathrm{H} & 1.30592700 & 2.05697400 & -1.66026400 \\ \mathrm{H} & -3.15134500 & 2.06701700 & 0.96289900 \\ \mathrm{H} & -5.18852800 & 0.69293600 & 0.96659700 \\ \mathrm{H} & -3.14990700 & -2.34239400 & -1.26154700 \\ \mathrm{H} & -1.11219900 & -0.98885200 & -1.25615100 \\ \mathrm{H} & 6.13457100 & 0.20665200 & -0.19520300 \\ \mathrm{H} & 4.92749600 & 0.56455000 & -1.45387700 \\ \mathrm{H} & 5.71791200 & -1.02996900 & -1.39846500 \\ \mathrm{H} & 5.02055200 & -2.60364600 & 0.46797500 \\ \mathrm{H} & 5.39872300 & -1.37564100 & 1.69236500 \\ \mathrm{H} & 3.75006100 & -2.03657100 & 1.57965000 \\ \mathrm{H} & -5.45956300 & -2.68183500 & 0.48804500 \\ \mathrm{H} & -5.83181700 & -2.13012100 & -1.14332800 \\ \mathrm{H} & -6.43801900 & -1.23245500 & 0.25136800 \\ \mathrm{H} & -0.81239200 & 2.30245200 & 1.56353500 \\ & & & \\ & & & \end{array}$<smiles></smiles>

\section{CBS-QB3 Enthalpy = -796.569996 CBS-QB3 Free Energy = -796.632690}

$$
\begin{aligned}
& \mathrm{O}^{1}{ }^{1} \\
& \mathrm{C} \\
& \mathrm{N} \\
& \mathrm{C} \\
& \mathrm{C} \\
& \mathrm{C} \\
& \mathrm{N} \\
& \mathrm{C} \\
& \mathrm{C} \\
& \mathrm{C} \\
& \mathrm{C} \\
& \mathrm{C} \\
& \mathrm{C} \\
& \mathrm{N}
\end{aligned}
$$

$$
\begin{array}{r}
2.08830700 \\
2.96477500 \\
2.66344200 \\
1.41296300 \\
0.42052100 \\
0.83760200 \\
-0.90114900 \\
-2.01645000 \\
-3.28464000 \\
-4.43492300 \\
-4.45792800 \\
-3.16670200 \\
-2.00858000 \\
4.24284100
\end{array}
$$

$$
\begin{array}{r}
-0.76350600 \\
-0.02161200 \\
0.63393200 \\
0.56872200 \\
-0.11455400 \\
-0.80320700 \\
-0.19768700 \\
-0.06196000 \\
-0.35188000 \\
-0.23537300 \\
0.20661900 \\
0.53449200 \\
0.40489500 \\
0.06437800
\end{array}
$$




$\begin{array}{lrrr}\mathrm{C} & 4.67974600 & -1.79817300 & -0.62906200 \\ \mathrm{C} & 5.27077300 & 0.12077300 & 0.80379000 \\ \mathrm{C} & -5.61388800 & -1.64031900 & 0.31754700 \\ \mathrm{O} & -0.94105200 & 2.53614600 & -0.40609700 \\ \mathrm{H} & 1.16265600 & 2.35976000 & 1.06116900 \\ \mathrm{H} & 0.14570700 & -0.98182100 & -1.42374900 \\ \mathrm{H} & -3.26818600 & 2.17659000 & -0.67564800 \\ \mathrm{H} & -5.38059500 & 0.91850100 & -0.47878900 \\ \mathrm{H} & -3.14741800 & -2.53972000 & 0.91275400 \\ \mathrm{H} & -1.07554900 & -1.29745500 & 0.69627600 \\ \mathrm{H} & 3.83315800 & -2.25946200 & -1.12718600 \\ \mathrm{H} & 5.44613800 & -1.55027500 & -1.37279400 \\ \mathrm{H} & 5.11405100 & -2.50517300 & 0.08626000 \\ \mathrm{H} & 6.06049200 & 0.46095800 & 0.12364200 \\ \mathrm{H} & 4.83040700 & 0.97969900 & 1.29961300 \\ \mathrm{H} & 5.72378900 & -0.54125800 & 1.54975800 \\ \mathrm{H} & -6.57010700 & -1.18991800 & 0.08013400 \\ \mathrm{H} & -5.61982300 & -2.67241100 & 0.64701400\end{array}$<smiles>Cc1ccc(Nc2cnc(N(C)C)nc2)cc1</smiles>

\section{CBS-QB3 Enthalpy $=-722.705239$ \\ CBS-QB3 Free Energy $=-722.771337$ \\ DFT Enthalpy $=-723.918459$ \\ DFT Free Energy = -723.984290}

3.26912500
4.49523200
4.46038700
3.26156500
2.03315200
2.05542700
0.84597300
-0.45683100
-1.35565900
-2.60713700
-2.99014100
-2.20394300
-0.97196800
-4.26106700
-5.20411800
-4.78666400
5.80548200
3.25372500
5.39181400
3.26880000
1.12779200
0.92880800
-1.04848600

1.26688600

0.63752100

$-0.67790500$

$-1.32641500$

$-0.68318300$

0.62832800

$-1.37191500$

$-0.86963600$

$-1.44617700$

$-1.03150600$

0.03141200

0.66448800

0.20196900

0.50527400

$-0.14637600$

1.60245400

1.34722600

2.28280900

$-1.20684000$

$-2.34292400$

1.14717700

$-2.37692400$

$-2.29496300$
0.49473000

0.27480600

$-0.20333300$

$-0.46239200$

$-0.23878500$

0.25099400

$-0.52386800$

$-0.32121100$

0.57826600

0.73130700

$-0.00623000$

$-0.90582300$

$-1.05884700$

0.16951400

1.06176100

$-0.62442100$

0.51914900

0.87746700

$-0.38167400$

$-0.84445700$

0.45653300

$-0.51831600$

1.18758800 


$\begin{array}{rrrr}\mathrm{H} & -0.35155800 & 0.70009000 & -1.79996700 \\ \mathrm{H} & -4.69111000 & -0.91119600 & 1.63639700 \\ \mathrm{H} & -6.02015900 & -0.61159400 & 0.49340300 \\ \mathrm{H} & -5.64268800 & 0.59042400 & 1.74380900 \\ \mathrm{H} & -3.98273200 & 2.05440300 & -1.19696400 \\ \mathrm{H} & -5.56238600 & 1.24762100 & -1.31566100 \\ \mathrm{H} & -5.23615900 & 2.35497900 & 0.03277500 \\ \mathrm{H} & 5.67001600 & 2.21837200 & 1.16425400 \\ \mathrm{H} & 6.53531400 & 0.68789900 & 0.99743100 \\ \mathrm{H} & 6.25228200 & 1.69965300 & -0.41744900\end{array}$<smiles>CN(C)c1ncc(Nc2ccccc2)cn1</smiles>

\section{CBS-QB3 Enthalpy $=-683.476910$ CBS-QB3 Free Energy $=-683.538003$ DFT Enthalpy $=-683.998120$ DFT Free Energy $=-684.056501$}

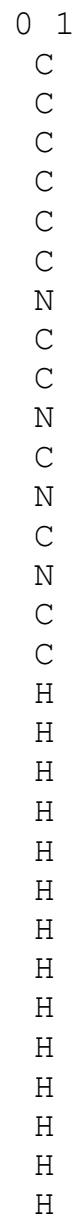

\author{
3.55964900 \\ 4.82079900 \\ 4.91134900 \\ 3.76643400 \\ 2.49177900 \\ 2.40300400 \\ 1.36442800 \\ 0.02365600 \\ $-0.83583800$ \\ $-2.11474200$ \\ $-2.56968200$ \\ $-1.82541700$ \\ $-0.56202100$ \\ $-3.87362400$ \\ $-4.76959700$ \\ $-4.46953300$ \\ 3.46707300 \\ 5.71545900 \\ 5.88305300 \\ 3.85027700 \\ 1.43300900 \\ 1.51863500 \\ $-0.47058300$ \\ 0.02739000 \\ $-5.23335300$ \\ $-4.21100800$ \\ $-5.57005800$ \\ $-4.94380400$ \\ $-5.23962900$ \\ $-3.70096900$
}

1.53392600

0.99808000

$-0.24409100$

$-0.93142700$

$-0.39374100$

0.85181900

$-1.11181200$

$-0.72661300$

$-1.43596400$

$-1.12569400$

$-0.03888700$

0.71944300

0.36031700

0.32269300

$-0.45385100$

1. 44870500

2.49515800

1.53511200

$-0.68215000$

$-1.89188300$

1.28010200

$-2.09628400$

$-2.30480600$

0.95927500

0.19353800

$-1.24164300$

$-0.90336700$

2.12596200

1.10531400

1.98361400
0.80960400

0.56254800

$-0.06552000$

$-0.44554400$

$-0.19709100$

0.44191700

$-0.60944300$

$-0.38647200$

0.45440800

0.62440300

$-0.03459100$

$-0.87139400$

$-1.04367100$

0.15719300

0.99679000

$-0.54137400$

1.30362500

0.85361700

$-0.26637700$

$-0.94516400$

0.65972000

$-0.76343500$

0.99979600

$-1.73390000$

1.74963400

1.49233700

0.39538800

0.17777000

$-1.24396200$

$-1.09036900$ 
<smiles>Cc1ccc(Nc2cnc(N(C)C)nc2)c(C)c1</smiles>

\section{CBS-QB3 Enthalpy $=-761.935713$ \\ CBS-QB3 Free Energy = -762.004954 \\ DFT Enthalpy $=-763.216452$ \\ DFT Free Energy $=-763.285398$}

O 1
C
C
C
C
C
C
$\mathrm{N}$
$\mathrm{C}$
$\mathrm{C}$
$\mathrm{N}$
$\mathrm{C}$
$\mathrm{N}$
$\mathrm{C}$
$\mathrm{N}$
$\mathrm{C}$
$\mathrm{C}$
$\mathrm{C}$
$\mathrm{H}$
$\mathrm{H}$
$\mathrm{H}$
$\mathrm{H}$
$\mathrm{H}$
$\mathrm{H}$
$\mathrm{H}$
$\mathrm{H}$
$\mathrm{H}$
$\mathrm{H}$
$\mathrm{H}$
$\mathrm{H}$
$\mathrm{H}$
$\mathrm{H}$
$\mathrm{H}$
$\mathrm{H}$
$\mathrm{C}$
$\mathrm{H}$
2.93471500
4.20175500
4.26695400
3.13437200
1.86098400
1.78051300
0.71702400
$-0.61061200$
$-1.49256400$
$-2.76448000$
$-3.18712200$
$-2.41943800$
$-1.16517300$
$-4.48120700$
$-5.40570100$
$-5.04825200$
5.45789700
2.83885900
5.24107400
0.83786900
$-1.15432900$
$-0.55795000$
$-5.90045300$
$-6.18123300$
$-4.86325300$
$-5.55200600$
$-4.25782700$
$-5.78845500$
5.24554400
6.20618100
5.91975400
0.81196700
3.25826200
2. 87550800
2.69774800
4.30153700

$-0.38354800$

$-0.24400900$

0.04095000

0.19846000

0.05206800

$-0.24510800$

0.23125100

0.12913300

$-0.86279100$

$-0.92683200$

0.00667800

1. 01024100

1.06415900

$-0.06758500$

$-1.06307100$

0.93250000

$-0.37331000$

$-0.61508000$

0.15299600

$-0.00845100$

$-1.62896700$

1. 88522100

$-1.57653000$

$-0.59285800$

$-1.78631300$

0.44079600

1.57422100

1.54911300

$-0.82357200$

$-0.99553500$

0.60315700

$-0.38083900$

0.52294000

$-0.28606500$

1. 42807900

0.68079600 
<smiles>Cc1ccccc1Nc1cnc(N(C)C)nc1</smiles>

CBS-QB3 Enthalpy $=-722.707190$

CBS-QB3 Free Energy = -722.771051

DFT Enthalpy $=-723.918741$

DFT Free Energy $=-723.982337$

01

C

C

C

C

C

C

$\mathrm{N}$

C

C

N

C

$\mathrm{N}$

C

N

C

C

$\mathrm{H}$

$\mathrm{H}$

$\mathrm{H}$

$\mathrm{H}$

$\mathrm{H}$

$\mathrm{H}$

$\mathrm{H}$

$\mathrm{H}$

$\mathrm{H}$

$\mathrm{H}$

$\mathrm{H}$

$\mathrm{H}$

$\mathrm{H}$

$\mathrm{H}$
$\mathrm{H}$

\begin{abstract}
3.11599900
4.42040300

4.63001800

3.57221900

2. 24985300

2.03859400

1.18384100

$-0.18286400$

$-1.02278900$

$-2.32213400$

$-2.82050200$

$-2.09766500$

$-0.81137900$

$-4.14873200$

$-5.02092200$

$-4.78771500$

2. 92755800

5.26263200

5.64404800

1. 38844400

$-0.62392900$

$-0.23766600$

$-5.78475700$

$-5.53283500$

$-4.43313200$

$-5.52678100$

$-5.30849200$

$-4.03716100$

3.82609500

3.32396000

3. 46723000

4.89364700

1.02800500
\end{abstract}

2.06229700

1.59954700

0.27237200

$-0.60095000$

$-0.11989200$

1.21185000

$-0.99266900$

$-0.64867000$

$-1.18957600$

$-0.92828400$

$-0.06109500$

0.52221500

0.21297900

0.25188900

$-0.35353600$

1.14346100

3.08921900

2.25759600

$-0.09936300$

$-1.97081000$

$-1.87858600$

0.66730200

$-0.97414500$

0.42562900

$-0.97166300$

0.60027800

1. 94830400

1.57042800

$-2.02754000$

$-2.26476200$

$-2.74827000$

$-2.20947900$

1.57878500
0.59455700

0.45875700

0.08833800

$-0.15688700$

$-0.01400200$

0.36669900

$-0.28331800$

$-0.17044300$

0.80401700

0.87796000

$-0.02794900$

$-1.01126700$

$-1.07536900$

0.05258000

1.04405300

$-0.90001900$

0.88785200

0.63653300

$-0.02186500$

$-0.15095100$

1.54704900

$-1.87973300$

0.55848300

1.62034900

1.71536800

$-1.50268700$

$-0.36884100$

$-1.55772500$

$-0.57444900$

$-1.51892400$

0.17264700

$-0.70600700$

0.49295200<smiles>Cc1cc(C)c(Nc2cnc(N(C)C)nc2)c(C)c1</smiles> 


CBS-QB3 Enthalpy $=-801.164556$
CBS-QB3 Free Energy $=-801.236835$
DFT Enthalpy $=-802.511129$
DFT Free Energy $=-802.583085$

O 1
$\mathrm{C}$
$\mathrm{C}$
$\mathrm{C}$
$\mathrm{C}$
$\mathrm{C}$
$\mathrm{C}$
$\mathrm{N}$
$\mathrm{C}$
$\mathrm{C}$
$\mathrm{N}$
$\mathrm{C}$
$\mathrm{N}$
$\mathrm{C}$
$\mathrm{N}$
$\mathrm{C}$
$\mathrm{C}$
$\mathrm{C}$
$\mathrm{C}$
$\mathrm{C}$
$\mathrm{H}$
$\mathrm{H}$
$\mathrm{H}$
$\mathrm{H}$
$\mathrm{H}$
$\mathrm{H}$
$\mathrm{H}$
$\mathrm{H}$
$\mathrm{H}$
$\mathrm{H}$
$\mathrm{H}$
$\mathrm{H}$
$\mathrm{H}$
$\mathrm{H}$
$\mathrm{H}$
$\mathrm{H}$
$\mathrm{H}$
$\mathrm{H}$
$\mathrm{H}$
$\mathrm{H}$
$\mathrm{H}$
$\mathrm{H}$

$\begin{array}{rr}3.33099500 & -1.34766500 \\ 4.20703000 & -0.70857700 \\ 3.86158000 & 0.56441500 \\ 2.67411700 & 1.18869500 \\ 1.80754300 & 0.52050000 \\ 2.14441500 & -0.75512800 \\ 0.61640100 & 1.15686100 \\ -0.67260000 & 0.70932500 \\ -1.81359700 & 1.27879800 \\ -3.05013800 & 0.91040200 \\ -3.18814000 & -0.09552600 \\ -2.16309400 & -0.71153800 \\ -0.93385300 & -0.30369300 \\ -4.46119100 & -0.53263900 \\ -5.62290600 & 0.19026400 \\ -4.70242200 & -1.49520700 \\ 5.50425900 & -1.36060700 \\ 1.26650700 & -1.46006900 \\ 2.32940200 & 2.55984900 \\ 3.58694400 & -2.33230200 \\ 4.52599900 & 1.08306800 \\ 0.68591000 & 2.16283700 \\ -1.71091300 & 2.07908500 \\ -0.11767600 & -0.80351400 \\ -6.40758000 & -0.52308200 \\ -5.34927700 & 0.77518500 \\ -6.02707000 & 0.86986500 \\ -3.80322900 & -2.07891100 \\ -5.51993100 & -2.16009900 \\ -4.98537000 & -1.00066300 \\ 5.38573400 & -2.43949100 \\ 5.87326200 & -0.94794500 \\ 6.28449800 & -1.20616000 \\ 1.82068500 & -2.25301500 \\ 0.38798800 & -1.91560400 \\ 0.89535500 & -0.75723600 \\ -1.32623700 & 2.86312900 \\ -36254000 & 3.32732400\end{array}$

$-0.48169300$

0.39696200

0.84996200

0.46560800

$-0.42042500$

$-0.91522100$

$-0.86248800$

$-0.50130200$

$-1.07808500$

$-0.77620100$

0.11264000

0.72910600

0.42540900

0.39025200

$-0.09661800$

1. 45071700

0.81261200

$-1.91783100$

0.99786400

$-0.86249800$

1. 53494900

$-0.90846000$

$-1.80997400$

0.93974500

$-0.36600800$

$-0.96971700$

0.66799000

1.62499700

1.15658300

2.39150400

0.94076100

1. 75434900

0.05892700

$-2.42327600$

$-1.44889700$

$-2.66755900$

1. 77004300

1. 43378800

0.21448200<smiles>CN(C)c1ncc(Nc2ccc(C(C)(C)C)cc2)cn1</smiles>

DFT Enthalpy $=-841.798699$ 


\section{DFT Free Energy $=-841.871642$}

$\begin{array}{lrrr}\mathrm{O}_{1} & & \\ \mathrm{C} & 2.32873200 & 0.78557400 & 0.22738200 \\ \mathrm{C} & 3.48985900 & 0.02601200 & 0.02903700 \\ \mathrm{C} & 3.29995000 & -1.31594600 & -0.31950900 \\ \mathrm{C} & 2.03115500 & -1.86462200 & -0.47205800 \\ \mathrm{C} & 0.88075700 & -1.09127100 & -0.26973500 \\ \mathrm{C} & 1.05268500 & 0.25298200 & 0.08943300 \\ \mathrm{~N} & -0.37790700 & -1.67936600 & -0.44308200 \\ \mathrm{C} & -1.61848700 & -1.03294100 & -0.26386100 \\ \mathrm{C} & -2.54767900 & -1.44137100 & 0.69508300 \\ \mathrm{~N} & -3.74808200 & -0.89240000 & 0.83304000 \\ \mathrm{C} & -4.04231700 & 0.14016700 & 0.01595100 \\ \mathrm{~N} & -3.22031000 & 0.61507900 & -0.94666700 \\ \mathrm{C} & -2.04399100 & 0.02053800 & -1.08072500 \\ \mathrm{~N} & -5.25459200 & 0.75367500 & 0.17407900 \\ \mathrm{C} & -6.23810300 & 0.26845500 & 1.12674100 \\ \mathrm{C} & -5.69201700 & 1.82259000 & -0.70723200 \\ \mathrm{C} & 4.87762100 & 0.66850600 & 0.19612800 \\ \mathrm{C} & 5.03148800 & 1.20472800 & 1.63847600 \\ \mathrm{C} & 5.03009800 & 1.84016000 & -0.80167800 \\ \mathrm{C} & 6.01776400 & -0.33258000 & -0.06536400 \\ \mathrm{H} & 2.41037500 & 1.83000200 & 0.50805900 \\ \mathrm{H} & 4.15211500 & -1.96223300 & -0.48590900 \\ \mathrm{H} & 1.92946400 & -2.90748900 & -0.75769700 \\ \mathrm{H} & 0.19010200 & 0.88090400 & 0.27334700 \\ \mathrm{H} & -0.39291500 & -2.68419700 & -0.36122500 \\ \mathrm{H} & -2.31074200 & -2.26423300 & 1.36797900 \\ \mathrm{H} & -1.39661800 & 0.39157600 & -1.87159900 \\ \mathrm{H} & -7.09729900 & -0.17760000 & 0.60850300 \\ \mathrm{H} & -5.78395500 & -0.48040700 & 1.76809900 \\ \mathrm{H} & -6.60490100 & 1.09991500 & 1.73835600 \\ \mathrm{H} & -4.85753000 & 2.15615600 & -1.31614900 \\ \mathrm{H} & -6.50068200 & 1.48051800 & -1.36667500 \\ \mathrm{H} & -6.07041000 & 2.66125900 & -0.11289700 \\ \mathrm{H} & 4.93650000 & 0.39457600 & 2.36662000 \\ \mathrm{H} & 4.27309100 & 1.95495000 & 1.87421600 \\ \mathrm{H} & 6.01352500 & 1.66987600 & 1.77156000 \\ \mathrm{H} & 4.27180200 & 2.60994000 & -0.64026800 \\ \mathrm{H} & 4.93342900 & 1.48807400 & -1.83233600 \\ \mathrm{H} & 6.01233600 & 2.31111800 & -0.69254000 \\ \mathrm{H} & 5.98470900 & -0.72874400 & -1.08404500 \\ \mathrm{H} & & & \end{array}$<smiles>CN(C)c1ncc(Nc2ccc(CC(C)(C)C)cc2)cn1</smiles>

\footnotetext{
DFT Enthalpy $=-881.096579$ DFT Free Energy $=-881.173204$
} 


\begin{tabular}{|c|c|c|c|}
\hline $\mathrm{C}$ & 1.93760600 & -055556600 & \\
\hline $\mathrm{C}$ & 3.06865900 & 0.26367000 & 0.67917600 \\
\hline $\mathrm{C}$ & 2.85247500 & 1.61399100 & 0.37485500 \\
\hline $\mathrm{C}$ & 1.57990800 & 2.11730900 & 0.14274800 \\
\hline $\mathrm{C}$ & 0.45443800 & 1.27986800 & 0.19998400 \\
\hline $\mathrm{C}$ & 0.65440500 & -0.07208700 & 0.50863300 \\
\hline $\mathrm{N}$ & -0.81377300 & 1.82985400 & -0.02509600 \\
\hline $\mathrm{C}$ & -2.02338600 & 1.10136600 & -0.05099300 \\
\hline $\mathrm{C}$ & -2.78619800 & 0.94492800 & -1.20976400 \\
\hline $\mathrm{N}$ & -3.95658300 & 0.32003900 & -1.23751200 \\
\hline $\mathrm{C}$ & -4.38757700 & -0.21576600 & -0.07610500 \\
\hline $\mathrm{N}$ & -3.73045000 & -0.12430200 & 1.10229900 \\
\hline $\mathrm{C}$ & -2.58217600 & 0.53639600 & 1.10019800 \\
\hline $\mathrm{N}$ & -5.57055800 & -0.90075200 & -0.09058200 \\
\hline $\mathrm{C}$ & -6.37826900 & -1.01068300 & -1.29304400 \\
\hline $\mathrm{C}$ & -6.14300500 & -1.47051100 & 1.11704200 \\
\hline C & 4.45798200 & -0.27670800 & 0.93935000 \\
\hline $\mathrm{C}$ & 5.25625200 & -0.82297600 & -0.28690100 \\
\hline C & 6.62473800 & -1.30754700 & 0.22785900 \\
\hline $\mathrm{C}$ & 4.51698700 & -2.00431200 & -0.93872600 \\
\hline $\mathrm{C}$ & 5.47569200 & 0.28273500 & -1.33397100 \\
\hline $\mathrm{H}$ & 2.05620100 & -1.60548800 & 0.99615900 \\
\hline $\mathrm{H}$ & 3.69810900 & 2.29283100 & 0.33105200 \\
\hline $\mathrm{H}$ & 1.44799900 & 3.17334700 & -0.07335400 \\
\hline $\mathrm{H}$ & -0.18973700 & -0.74801600 & 0.55895300 \\
\hline $\mathrm{H}$ & -0.81408300 & 2.69274800 & -0.54698000 \\
\hline $\mathrm{H}$ & -2.43678000 & 1.36222600 & -2.15299300 \\
\hline $\mathrm{H}$ & -2.06812600 & 0.62710200 & 2.05414500 \\
\hline $\mathrm{H}$ & -7.32868000 & -0.47532800 & -1.17123700 \\
\hline $\mathrm{H}$ & -6.60534700 & -2.06316600 & -1.49700500 \\
\hline $\mathrm{H}$ & -5.83700800 & -0.58820100 & -2.13375400 \\
\hline $\mathrm{H}$ & -7.07061900 & -0.95079300 & 1.38950500 \\
\hline $\mathrm{H}$ & -6.38143400 & -2.52763000 & 0.95456400 \\
\hline $\mathrm{H}$ & -5.43294700 & -1.38044300 & 1.93310400 \\
\hline $\mathrm{H}$ & 4.39163100 & -1.08457200 & 1.67700300 \\
\hline $\mathrm{H}$ & 5.06320400 & 0.51044500 & 1.40364900 \\
\hline $\mathrm{H}$ & 7.23240200 & -1.70407600 & -0.59130900 \\
\hline $\mathrm{H}$ & 7.18445500 & -0.49029500 & 0.69376500 \\
\hline $\mathrm{H}$ & 6.50897700 & -2.10146900 & 0.97248900 \\
\hline $\mathrm{H}$ & 5.09945700 & -2.40708400 & -1.77317600 \\
\hline $\mathrm{H}$ & 3.54136000 & -1.70223000 & -1.32659500 \\
\hline $\mathrm{H}$ & 4.35884600 & -2.81662900 & -0.22178900 \\
\hline $\mathrm{H}$ & 6.06960400 & -0.09562400 & -2.17176300 \\
\hline $\mathrm{H}$ & 6.01316000 & 1.13442400 & -0.90413500 \\
\hline $\mathrm{H}$ & 4.52771300 & 0.64941100 & -1.73459200 \\
\hline
\end{tabular}<smiles>CCc1ccc(Nc2cnc(N(C)C)nc2)cc1</smiles>

CBS-QB3 Enthalpy = -761.930041 CBS-QB3 Free Energy = -761.998564 
DFT Enthalpy $=-763.212729$

DFT Free Energy $=-763.280962$

$\begin{array}{lrrr}\mathrm{O}^{1} & & & \\ \mathrm{C} & 2.92321200 & -1.13042500 & 0.07928800 \\ \mathrm{C} & 4.11695600 & -0.41744200 & 0.21156400 \\ \mathrm{C} & 4.01681100 & 0.96754400 & 0.38714000 \\ \mathrm{C} & 2.78654700 & 1.60835200 & 0.43522400 \\ \mathrm{C} & 1.59278100 & 0.88210400 & 0.29654100 \\ \mathrm{C} & 1.68030200 & -0.50432600 & 0.11439600 \\ \mathrm{~N} & 0.37130500 & 1.56526200 & 0.36716400 \\ \mathrm{C} & -0.90157800 & 0.97656100 & 0.20620800 \\ \mathrm{C} & -1.74821800 & 1.28850900 & -0.85919000 \\ \mathrm{~N} & -2.97417600 & 0.79701300 & -0.98911600 \\ \mathrm{C} & -3.38116200 & -0.07901400 & -0.04681200 \\ \mathrm{~N} & -2.64495300 & -0.45098300 & 1.02470100 \\ \mathrm{C} & -1.43955800 & 0.08608900 & 1.14157300 \\ \mathrm{~N} & -4.62377000 & -0.63277700 & -0.18520700 \\ \mathrm{C} & -5.51636600 & -0.25046600 & -1.26567400 \\ \mathrm{C} & -5.17539200 & -1.53725800 & 0.80888700 \\ \mathrm{C} & 5.46190400 & -1.10515000 & 0.12937600 \\ \mathrm{H} & 2.95909600 & -2.20701700 & -0.05899000 \\ \mathrm{H} & 4.92101800 & 1.55839100 & 0.49987400 \\ \mathrm{H} & 2.74172000 & 2.68190800 & 0.59259300 \\ \mathrm{H} & 0.78029400 & -1.09381000 & -0.00592600 \\ \mathrm{H} & 0.42466900 & 2.54951500 & 0.15423400 \\ \mathrm{H} & -1.42019900 & 1.98336700 & -1.63090700 \\ \mathrm{H} & -0.86018200 & -0.19841500 & 2.01666900 \\ \mathrm{H} & -6.38612800 & 0.29501900 & -0.87687400 \\ \mathrm{H} & -5.88039300 & -1.14484500 & -1.78340300 \\ \mathrm{H} & -4.98490500 & 0.38344800 & -1.96852800 \\ \mathrm{H} & -6.01209000 & -1.06713400 & 1.34198700 \\ \mathrm{H} & -5.55043700 & -2.44330400 & 0.32026600 \\ \mathrm{H} & -4.40448800 & -1.80389700 & 1.52508200 \\ \mathrm{H} & 5.37217500 & -2.12543600 & 0.51665600 \\ \mathrm{H} & 6.17433200 & -0.59028100 & 0.78280600 \\ \mathrm{C} & 6.03325200 & -1.15585800 & -1.29842000 \\ \mathrm{H} & 5.35947500 & -1.69518900 & -1.96976800 \\ \mathrm{H} & 7.00482800 & -1.65869100 & -1.31629500 \\ \mathrm{H} & 6.16543800 & -0.14855200 & -1.70287200 \\ & & & \\ & & & \\ & & & \\ & & & \end{array}$<smiles>CN(C)c1ncc(Nc2ccccc2)cn1</smiles>

CBS-QB3 Enthalpy $=-682.848903$

CBS-QB3 Free Energy $=-682.907514$

DFT Enthalpy $=-683.998120$

DFT Free Energy $=-684.056501$ 
02

$\begin{array}{lr}\mathrm{C} & -3.74176300 \\ \mathrm{C} & -4.88697800 \\ \mathrm{C} & -4.82045400 \\ \mathrm{C} & -3.62820100 \\ \mathrm{C} & -2.44372100 \\ \mathrm{C} & -2.53457100 \\ \mathrm{~N} & -1.31984800 \\ \mathrm{C} & -0.06616000 \\ \mathrm{C} & 1.00510900 \\ \mathrm{~N} & 2.27276800 \\ \mathrm{C} & 2.55792100 \\ \mathrm{~N} & 1.64198400 \\ \mathrm{C} & 0.37225700 \\ \mathrm{~N} & 3.86426100 \\ \mathrm{C} & 4.92745700 \\ \mathrm{C} & 4.28088100 \\ \mathrm{H} & -3.79354800 \\ \mathrm{H} & -5.82406100 \\ \mathrm{H} & -5.70912400 \\ \mathrm{H} & -3.56246200 \\ \mathrm{H} & -1.66683900 \\ \mathrm{H} & 0.76016300 \\ \mathrm{H} & -0.34152200 \\ \mathrm{H} & 5.48363700 \\ \mathrm{H} & 4.49955300 \\ \mathrm{H} & 5.62791700 \\ \mathrm{H} & 4.99329800 \\ \mathrm{H} & 4.77532800 \\ \mathrm{H} & 3.41284500 \\ & \end{array}$<smiles>Cc1ccc(Nc2cnc(N(C)C)nc2)c(C)c1</smiles>

1.48753900
0.94353600
-0.31876900
-1.02356200
-0.46995900
0.80307600
-1.25854600
-0.75972500
-1.64435300
-1.29687700
-0.01708200
0.89407100
0.53212100
0.36909300
-0.54102600
1.71026500
2.45150300
1.48738700
-0.75645600
-2.01121200
1.22091100
-2.66995400
1.26378000
-0.12089300
-1.49698100
-0.68813700
1.66152900
2.20350400
2.28846900

$-0.68094700$

$-0.09718600$

0.49936200

0.51575600

$-0.02778900$

$-0.64488400$

0.00942600

0.02466800

$-0.28924600$

$-0.29253900$

0.05607900

0.45417600

0.43165200

0.02219000

$-0.37372600$

0.39981100

$-1.17553800$

$-0.12381900$

0.94063000

0.95587100

$-1.14014700$

$-0.55369000$

0.79693500

$-1.21916600$

$-0.65761000$

0.45661700

1. 23120600

$-0.44488300$

0.69953500
CBS-QB3 Enthalpy $=-761.308886$

CBS-QB3 Free Energy $=-761.376437$

DFT Enthalpy $=-762.596294$

DFT Free Energy $=-762.663557$
${ }^{0}{ }^{2}$
C
C
C
C
C
C
N
C
C
N

\author{
3.10517700 \\ 4. 28340200 \\ 4. 21082500 \\ 3.02672000 \\ 1.81850000 \\ 1.90049500 \\ 0.68735900 \\ $-0.56420700$ \\ $-1.64496100$ \\ $-2.90868400$
}

$-1.59620400$

$-0.97835200$

0.36532200

1.08958600

0.43924200

$-0.91224500$

1. 20560000

0.69804600

1. 50130500

1.13865000
$-0.56085600$

$-0.12707300$

0.26906500

0.25689800

$-0.14552700$

$-0.56543100$

$-0.19354200$

$-0.10553700$

$-0.56274600$

$-0.51173600$ 


$\begin{array}{lrrr}\mathrm{C} & -3.17705200 & -0.06894600 & 0.04310800 \\ \mathrm{~N} & -2.24997300 & -0.88671500 & 0.58866400 \\ \mathrm{C} & -0.98490600 & -0.51344700 & 0.50777800 \\ \mathrm{~N} & -4.47860400 & -0.47565700 & 0.07081300 \\ \mathrm{C} & -5.55255500 & 0.33978200 & -0.47291000 \\ \mathrm{C} & -4.87697600 & -1.74102400 & 0.66543100 \\ \mathrm{C} & 5.59122700 & -1.72676300 & -0.08088900 \\ \mathrm{H} & 3.13666900 & -2.62269900 & -0.91235500 \\ \mathrm{H} & 5.12056500 & 0.86556500 & 0.58964300 \\ \mathrm{H} & -1.41581000 & 2.47311500 & -0.99282500 \\ \mathrm{H} & -0.26154200 & -1.16288900 & 0.99099800 \\ \mathrm{H} & -6.09837600 & -0.22181800 & -1.23954900 \\ \mathrm{H} & -6.26024300 & 0.61181300 & 0.31914300 \\ \mathrm{H} & -5.13699700 & 1.24227500 & -0.90940300 \\ \mathrm{H} & -5.36009500 & -2.37656300 & -0.08581700 \\ \mathrm{H} & -4.00169400 & -2.24771300 & 1.05909500 \\ \mathrm{H} & -5.59391800 & -1.56646800 & 1.47605000 \\ \mathrm{H} & 5.56193700 & -2.62065400 & -0.70760800 \\ \mathrm{H} & 6.42188600 & -1.10162400 & -0.41967000 \\ \mathrm{H} & 5.82528800 & -2.04881300 & 0.94042900 \\ \mathrm{H} & 1.01498800 & -1.39880700 & -0.95537300 \\ \mathrm{C} & 2.99363800 & 2.53349300 & 0.67846700 \\ \mathrm{H} & 2.46320800 & 3.13773700 & -0.06108400 \\ \mathrm{H} & 2.45315700 & 2.65990100 & 1.62172800 \\ \mathrm{H} & 4.00432800 & 2.92572000 & 0.80854400\end{array}$<smiles>Cc1ccccc1Nc1cnc(N(C)C)nc1</smiles>

CBS-QB3 Enthalpy $=-722.079443$

CBS-QB3 Free Energy = -722.141767

DFT Enthalpy $=-723.297416$

DFT Free Energy = -723.359481

O $^{2}$
$C$
$C$
$C$
$C$
$C$
$C$
N
C
C
N
C
N
C
N
C
C

\begin{abstract}
$-3.34188600$
$-4.54416000$

$-4.59070700$

$-3.46410400$

$-2.22101500$

$-2.19631100$

$-1.14959300$

0.13376800

1.15548600

2.44184700

2.79577100

1.92802500

0.63972800

4.12343200

5.13800500

4.61120100
\end{abstract}

-1.97859300
-1.44565200
-0.11649000
0.69896700
0.14822800
-1.19821800
1.00108100
0.59254000
1.47638800
1.21103300
0.02691700
-0.86036900
-0.58519000
-0.27867300
0.61633400
-1.51131200

0.60596700

0.14120800

$-0.28325700$

$-0.26415700$

0.17352600

0.61736100

0.23115800

0.12899000

0.57699600

0.51248100

$-0.04620500$

$-0.58209900$

$-0.48886000$

$-0.08847100$

0.44498900

$-0.68612600$ 
$-2.99919800$

$-2.05222700$

0.29947400

2.42742300

$-1.28903400$

0.93942500

0.09791200

1.48541400

$-1.28314300$

$-2.10749000$

$-2.08292200$

2.13257600

2.27897100

2.78834600

2.44803800

$-1.60146000$
0.97038000
0.12601200
$-0.62997500$
1.01083500
$-0.96281000$
$-0.35416800$
1. 20645500
0.88498600
$-1.50212500$
0.06166100
$-1.07260800$
$-0.71567700$
$-1.65908600$
0.01425300
$-0.85941100$
1.02558500<smiles>Cc1cc(C)c(Nc2cnc(N(C)C)nc2)c(C)c1</smiles>

\section{CBS-QB3 Enthalpy $=-800.535370$ \\ CBS-QB3 Free Energy = -800.606289 \\ DFT Enthalpy $=-801.888844$ \\ DFT Free Energy $=-801.959446$}

\author{
3.26726800 \\ 4.24459800 \\ 3.97619400 \\ 2.77467800 \\ 1.76377600 \\ 2.03257200 \\ 0.60629200 \\ $-0.61606900$ \\ $-1.75355500$ \\ $-2.99612300$ \\ $-3.18707900$ \\ $-2.19841100$ \\ $-0.95639700$ \\ $-4.47077100$ \\ $-5.60590500$ \\ $-4.78756400$ \\ 5.55105200 \\ 1.07405700 \\ 2.52079900 \\ 3. 47611400 \\ 4.73206400 \\ $-1.58513300$ \\ $-0.17277500$ \\ $-6.26627900$
}

-1.39727200
-0.71775500
0.60190800
1.22720600
0.49796000
-0.82862800
1.19503500
0.72738400
1.39200900
1.03354600
-0.02094700
-0.69475600
-0.33209600
-0.41824500
0.24364400
-1.52807300
-1.37872100
-1.60197100
2.65758400
-2.40416000
1.16428600
2.24142900
-0.85862000
0.66215400

$-0.44444800$

0.28191800

0.65824200

0.34776800

$-0.33424500$

$-0.76193000$

$-0.66182500$

$-0.37476900$

$-0.92283400$

$-0.69123800$

0.14166000

0.77345600

0.51801300

0.37040300

$-0.25312600$

1. 25527300

0.64705800

$-1.63956900$

0.74725500

$-0.79514300$

1. 19931600

$-1.58039100$

1.05565500

0.51506800 


$\begin{array}{rrrr}\mathrm{H} & -6.18271700 & -0.47766500 & -0.84301300 \\ \mathrm{H} & -5.25125300 & 1.04076800 & -0.89832000 \\ \mathrm{H} & -3.87190700 & -1.92362900 & 1.68268500 \\ \mathrm{H} & -5.30307800 & -2.31929000 & 0.69877800 \\ \mathrm{H} & -5.45076600 & -1.18829400 & 2.05873000 \\ \mathrm{H} & 6.38914000 & -0.68182100 & 0.56097300 \\ \mathrm{H} & 5.75767600 & -2.23684400 & 0.00359800 \\ \mathrm{H} & 5.53594800 & -1.74032200 & 1.68159700 \\ \mathrm{H} & 0.57371400 & -0.94778700 & -2.35713500 \\ \mathrm{H} & 0.29142900 & -2.10963300 & -1.06701300 \\ \mathrm{H} & 1.61447900 & -2.36791200 & -2.19955000 \\ \mathrm{H} & 2.10792800 & 3.22260800 & -0.09219200 \\ \mathrm{H} & 3.44136200 & 3.13708300 & 1.08634900 \\ \mathrm{H} & 1.78658800 & 2.72519000 & 1.55683600\end{array}$<smiles>CN(C)c1ncc(Nc2ccc(C(C)(C)C)cc2)cn1</smiles>

\section{DFT Enthalpy $=-841.176875$ \\ DFT Free Energy $=-841.247733$}

$\mathrm{O}^{2}$
$\mathrm{C}$
$\mathrm{C}$
$\mathrm{C}$
$\mathrm{C}$
$\mathrm{C}$
$\mathrm{C}$
$\mathrm{N}$
$\mathrm{C}$
$\mathrm{C}$
$\mathrm{N}$
$\mathrm{C}$
$\mathrm{N}$
$\mathrm{C}$
$\mathrm{N}$
$\mathrm{C}$
$\mathrm{C}$
$\mathrm{C}$
$\mathrm{C}$
$\mathrm{C}$
$\mathrm{C}$
$\mathrm{H}$
$\mathrm{H}$
$\mathrm{H}$
$\mathrm{H}$
$\mathrm{H}$
$\mathrm{H}$
$\mathrm{H}$
$\mathrm{H}$
$\mathrm{H}$
$\mathrm{H}$

$$
\begin{array}{rr}
2.41109700 & 0.70303200 \\
3.49012200 & -0.01632800 \\
3.20122300 & -1.28155200 \\
1.91447400 & -1.79701400 \\
0.82186300 & -1.05467800 \\
1.11552200 & 0.21578700 \\
-0.40576900 & -1.66352200 \\
-1.57304000 & -0.98220700 \\
-2.75730200 & -1.69094400 \\
-3.95988200 & -1.15913500 \\
-4.05844800 & 0.14122600 \\
-3.02526900 & 0.89710200 \\
-1.82252900 & 0.35035000 \\
-5.29289200 & 0.71836900 \\
-6.47123900 & -0.01480000 \\
-5.51234500 & 2.09870600 \\
4.90572800 & 0.58099300 \\
5.33134800 & 0.83447900 \\
4.91337800 & 1.92094900 \\
5.94674000 & -0.35096500 \\
2.58835300 & 1.67017100 \\
3.99353700 & -1.88808100 \\
1.70864100 & -2.78316500 \\
0.32782000 & 0.79071500 \\
-2.66276500 & -2.73626200 \\
-1.01563800 & 0.95974700 \\
-7.20418900 & -0.06902700 \\
-6.18505400 & -1.01935800 \\
-6.94003800 & 0.49738400 \\
-4.57430900 & 2.53374200
\end{array}
$$$$
0.56293100
$$

0.01803600

$-0.51573800$

$-0.51147200$

$-0.00974000$

0.54848600

$-0.02811900$

$-0.03659500$

0.31150800

0.32568700

$-0.04707800$

$-0.47988800$

$-0.46743300$

$-0.00187500$

0.43224200

$-0.40237600$

0.04571100

1.51136200

$-0.72741800$

$-0.60097700$

1.02028200

$-0.93450700$

$-0.91039900$

1.01961500

0.59466800

$-0.86212400$

$-0.38126100$

0.72651900

1.28010600

$-0.73191700$ 


$\begin{array}{rrrr}\mathrm{H} & -6.24232000 & 2.14126200 & -1.21887500 \\ \mathrm{H} & -5.90832300 & 2.67831700 & 0.43956600 \\ \mathrm{H} & 5.33753100 & -0.09805300 & 2.08199600 \\ \mathrm{H} & 4.65700400 & 1.53023400 & 2.01572600 \\ \mathrm{H} & 6.33837700 & 1.26177200 & 1.54550300 \\ \mathrm{H} & 4.22359700 & 2.64617600 & -0.28979400 \\ \mathrm{H} & 4.62287200 & 1.77052100 & -1.77067900 \\ \mathrm{H} & 5.91497300 & 2.36188100 & -0.71280700 \\ \mathrm{H} & 5.71605200 & -0.55166700 & -1.65071100 \\ \mathrm{H} & 6.93296600 & 0.11938300 & -0.56376400 \\ \mathrm{H} & 6.01803600 & -1.30758100 & -0.07647900\end{array}$<smiles>CN(C)c1ncc(Nc2ccc(CC(C)(C)C)cc2)cn1</smiles>

\section{DFT Enthalpy $=-880.474746$ DFT Free Energy $=-880.548970$}

$\begin{array}{lrrr}\mathrm{O}^{2} & & & \\ \mathrm{C} & 1.98986900 & -0.86067400 & -0.11744800 \\ \mathrm{C} & 3.02079500 & -0.05982900 & -0.63137900 \\ \mathrm{C} & 2.71284700 & 1.27941700 & -0.92386100 \\ \mathrm{C} & 1.44918100 & 1.79635600 & -0.70112900 \\ \mathrm{C} & 0.39661400 & 0.98302100 & -0.21544500 \\ \mathrm{C} & 0.70983200 & -0.36698700 & 0.08388600 \\ \mathrm{~N} & -0.81105900 & 1.59494700 & -0.00302000 \\ \mathrm{C} & -1.99058700 & 0.93514500 & -0.00925700 \\ \mathrm{C} & -3.11486200 & 1.57969100 & 0.57938400 \\ \mathrm{~N} & -4.32329300 & 1.06307500 & 0.62406900 \\ \mathrm{C} & -4.49274300 & -0.14911700 & 0.03968600 \\ \mathrm{~N} & -3.52833500 & -0.82367200 & -0.62455400 \\ \mathrm{C} & -2.31665200 & -0.29736700 & -0.64025500 \\ \mathrm{~N} & -5.73088500 & -0.71546400 & 0.10988600 \\ \mathrm{C} & -6.84036400 & -0.06191300 & 0.78557600 \\ \mathrm{C} & -6.02602300 & -1.99945700 & -0.50517200 \\ \mathrm{C} & 4.40736300 & -0.61569600 & -0.85957400 \\ \mathrm{C} & 5.42694800 & -0.50508000 & 0.32039700 \\ \mathrm{C} & 6.74984700 & -1.13590300 & -0.15334700 \\ \mathrm{C} & 4.91964300 & -1.26806100 & 1.55633800 \\ \mathrm{C} & 5.67634600 & 0.96634600 & 0.69423100 \\ \mathrm{H} & 2.19798400 & -1.89667400 & 0.13007100 \\ \mathrm{H} & 3.48475500 & 1.92502700 & -1.32967900 \\ \mathrm{H} & 1.22197800 & 2.83426700 & -0.91320900 \\ \mathrm{H} & -0.04479700 & -1.00922500 & 0.52071000 \\ \mathrm{H} & -2.96521000 & 2.55701500 & 1.03131900 \\ \mathrm{H} & -1.56962000 & -0.83265200 & -1.21789300 \\ \mathrm{H} & -7.64708700 & 0.14753600 & 0.07331100 \\ \mathrm{H} & -7.23875000 & -0.71577900 & 1.56953900 \\ \mathrm{H} & -6.49874400 & 0.86908400 & 1.22623500 \\ \mathrm{H} & -6.83403300 & -1.88819900 & -1.23735700 \\ \mathrm{H} & -6.35229900 & -2.71720400 & 0.25634700 \\ \mathrm{H} & -5.13727400 & -2.37651400 & -1.00085400 \\ & & & \end{array}$




$\begin{array}{rrrr}\mathrm{H} & 4.32392100 & -1.67543500 & -1.12551700 \\ \mathrm{H} & 4.85035200 & -0.11370300 & -1.72702300 \\ \mathrm{H} & 7.50779100 & -1.08802900 & 0.63431600 \\ \mathrm{H} & 7.14591100 & -0.61417500 & -1.03022100 \\ \mathrm{H} & 6.61332000 & -2.18811100 & -0.42212700 \\ \mathrm{H} & 5.65913600 & -1.22400900 & 2.36171500 \\ \mathrm{H} & 3.98716400 & -0.84429300 & 1.93647500 \\ \mathrm{H} & 4.74282500 & -2.32376400 & 1.32608900 \\ \mathrm{H} & 6.42450600 & 1.03593100 & 1.48975400 \\ \mathrm{H} & 6.05007700 & 1.53565600 & -0.16301100 \\ \mathrm{H} & 4.76476700 & 1.45168900 & 1.05054900\end{array}$<smiles>CCc1ccc(Nc2cnc(N(C)C)nc2)cc1</smiles>

\section{CBS-QB3 Enthalpy $=-761.302859$ \\ CBS-QB3 Free Energy = -761.369159 \\ DFT Enthalpy $=-762.591044$ \\ DFT Free Energy $=-762.657062$}

3.02555300
4.11666700
3.89917300
2.65257800
1.53496400
1.76277700
0.34935300
-0.85955700
-1.98083100
-3.21424200
-3.41171600
-2.44728900
-1.21171300
-4.67860000
-5.78956000
-5.00323100
5.49277500
3.17276300
4.72855600
2.48943700
0.95439400
-1.80754800
-0.46190000
-6.54431000
-6.26284600
-5.42657200
-5.76096300
-5.40914700
-4.10789800
5.39815900
6.02997400

$-0.31861600$

0.23394000

0.70093000

0.62157400

0.10699700

$-0.37897900$

0.04803700

0.04212000

$-0.38974900$

$-0.42647400$

0.00731200

0.52011800

0.52920800

$-0.05963100$

$-0.57652700$

0.40253600

0.26936300

$-0.71306500$

1. 12568400

0.96903800

$-0.84918000$

$-0.72129800$

0.98730400

0.20474600

$-1.41215700$

$-0.91402100$

1.19305300

$-0.42277800$

0.78783000

0.37992900

1. 15389800 


$\begin{array}{lrrr}\mathrm{C} & 6.32710500 & -0.70769200 & -0.98722700 \\ \mathrm{H} & 7.31496100 & -1.17284000 & -0.92380800 \\ \mathrm{H} & 6.46616500 & 0.36966400 & -1.10955300 \\ \mathrm{H} & 5.83231400 & -1.08214900 & -1.88726300\end{array}$<smiles>c1ccc(Nc2ccccc2)cc1</smiles>

CBS-QB3 Enthalpy $=-517.033358$

CBS-QB3 Free Energy= -517.081265

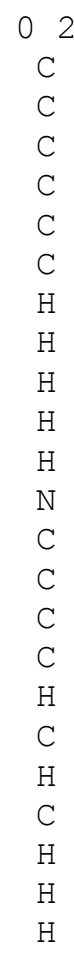<smiles>CON(c1ccccc1)c1ccccc1</smiles>

$$
\begin{array}{rr}
3.79683500 & 0.62683900 \\
3.59133700 & -0.65229000 \\
2.32381500 & -1.20693600 \\
1.20177100 & -0.48001100 \\
1.43464200 & 0.81014700 \\
2.71500500 & 1.34149400 \\
4.79284800 & 1.05357500 \\
4.43014100 & -1.21814200 \\
2.14435400 & -2.20333000 \\
0.61131600 & 1.35711300 \\
2.87701600 & 2.31877400 \\
-0.00002500 & -1.12657800 \\
-1.20184300 & -0.48008600 \\
-1.43469100 & 0.81008200 \\
-2.32377900 & -1.20695800 \\
-2.71500100 & 1.34148200 \\
-0.61125600 & 1.35689600 \\
-3.59128800 & -0.65225100 \\
-2.14431100 & -2.20331400 \\
-3.79680100 & 0.62685200 \\
-2.87701800 & 2.31874900 \\
-4.43010000 & -1.21801500 \\
-4.79283000 & 1.05355400
\end{array}
$$

$$
\begin{array}{r}
0.08738500 \\
-0.44003400 \\
-0.44977400 \\
0.02745800 \\
0.57615900 \\
0.60780900 \\
0.11117000 \\
-0.82971600 \\
-0.83478100 \\
1.01707400 \\
1.04937600 \\
-0.00022200 \\
-0.02754400 \\
-0.57630100 \\
0.44987500 \\
-0.60783400 \\
-1.01719300 \\
0.44022200 \\
0.83496900 \\
-0.08725600 \\
-1.04943100 \\
0.83002200 \\
-0.11092600
\end{array}
$$

CBS-QB3 Enthalpy= -707.039061

CBS-QB3 Free Energy= -707.097844
-3.61696000
-3.56391800
-2.36439800
-1.20293500
-1.25166500
-2.45802100
-4.55382500
-4.46083200
-2.31266100
-0.35899400

$-1.61933100$

$-0.61266400$

0.02678500

$-0.33437000$

$-1.33248200$

$-1.97317300$

$-2.11913300$

$-0.32826500$

0.80892300

$-1.59400900$
0.36953900

$-0.59472300$

$-0.88066400$

$-0.19063100$

0.78524200

1.05554300

0.58605700

$-1.13319500$

$-1.62638700$

1.33770600 


$\begin{array}{lrrr}\mathrm{H} & -2.49048100 & -2.74322000 & 1.81787900 \\ \mathrm{C} & 1.31549500 & -0.18072400 & -0.25318700 \\ \mathrm{C} & 1.60789000 & -1.50213700 & -0.60648300 \\ \mathrm{C} & 2.31254000 & 0.64534900 & 0.27149300 \\ \mathrm{C} & 2.89414200 & -1.99636900 & -0.41611100 \\ \mathrm{H} & 0.83873800 & -2.13030500 & -1.03813600 \\ \mathrm{C} & 3.59598000 & 0.13813300 & 0.44887500 \\ \mathrm{H} & 2.05947200 & 1.66034100 & 0.54489800 \\ \mathrm{C} & 3.89376400 & -1.18082400 & 0.11166700 \\ \mathrm{H} & 3.11672200 & -3.02000700 & -0.69531600 \\ \mathrm{H} & 4.36662600 & 0.77994900 & 0.86075900 \\ \mathrm{H} & 4.89531200 & -1.56919300 & 0.25510800 \\ \mathrm{~N} & 0.01271400 & 0.35046900 & -0.49774300 \\ \mathrm{O} & -0.07398800 & 1.61950900 & -0.75301500 \\ \mathrm{O} & -0.17012800 & 2.47489900 & 0.69415100 \\ \mathrm{C} & -0.64808700 & 3.72449000 & 0.29666700 \\ \mathrm{H} & -0.66032800 & 4.34130100 & 1.20459200 \\ \mathrm{H} & 0.01013700 & 4.21203200 & -0.43619800 \\ \mathrm{H} & -1.66893500 & 3.67694100 & -0.10601800\end{array}$

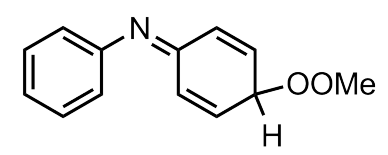

\section{CBS-QB3 Enthalpy $=-707.040615$ CBS-QB3 Free Energy= -707.099852}

$O^{O}{ }^{C}$
$C$
$C$
$C$
$C$
$C$
$C$
$C$
$H$
$H$
$H$
$H$
$H$
$H$
C
C
C
C
H
C
H
H
H
C
H
N
O
O
C

$$
\begin{array}{rr}
5.04449400 & -0.70852800 \\
4.67126600 & 0.43257200 \\
3.44817000 & 1.04570900 \\
2.55317500 & 0.50337800 \\
2.93904200 & -0.63877000 \\
4.17716400 & -1.23182300 \\
6.00621800 & -1.17480900 \\
5.34356400 & 0.85524900 \\
3.16022800 & 1.94618300 \\
2.27612000 & -1.03582100 \\
4.46423300 & -2.10718000 \\
0.19768400 & 0.63249900 \\
-0.09195600 & -0.73062700 \\
-0.96248800 & 1.44531600 \\
-1.33191700 & -1.23168900 \\
0.72842100 & -1.31263000 \\
-2.20069600 & 0.94540000 \\
-0.74810900 & 2.47599700 \\
-1.54421900 & -2.21925800 \\
-3.04698300 & 1.55339800 \\
-2.50365300 & -0.48690600 \\
-2.85771200 & -1.00949400 \\
1.35856500 & 1.18965000 \\
-3.55112800 & -0.60395600 \\
-4.79326400 & -0.30890000 \\
-5.71363000 & 0.09328100
\end{array}
$$

0.35073300

1.06182600

0.81978400

$-0.11596200$

$-0.83684100$

$-0.60536300$

0.53033900

1.80039800

1.34919800

$-1.59702700$

$-1.17786000$

$-0.31950500$

0.15918800

$-0.70958800$

0.14487900

0.55977600

$-0.75544300$

$-0.96747200$

0.54016100

$-1.05342400$

$-0.42090500$

$-1.32379900$

$-0.37333300$

0.55626700

$-0.16457300$

0.83202000 


$\begin{array}{lrrr}\mathrm{H} & -6.64208300 & 0.27968600 & 0.28778400 \\ \mathrm{H} & -5.87159600 & -0.70210400 & 1.56744100 \\ \mathrm{H} & -5.38284000 & 1.00720500 & 1.33533000\end{array}$<smiles>COC1C=CC=CC1=Nc1ccccc1</smiles>

CBS-QB3 Enthalpy= -707.033722

CBS-QB3 Free Energy= -707.092022

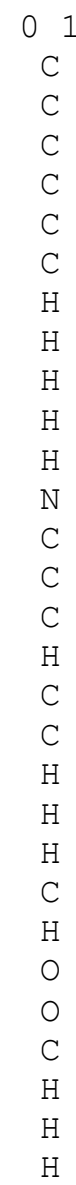<smiles>[O-]N(c1ccccc1)c1ccccc1</smiles>

$$
\begin{array}{rr}
-4.36952300 & -0.11978100 \\
-3.71308800 & 0.37174100 \\
-2.33366600 & 0.54640900 \\
-1.58027800 & 0.20213800 \\
-2.24448400 & -0.28482300 \\
-3.62838100 & -0.43902600 \\
-5.44629100 & -0.24247700 \\
-4.28019200 & 0.62948700 \\
-1.82069100 & 0.94325500 \\
-1.67050700 & -0.51860600 \\
-4.12789500 & -0.81066800 \\
-0.19733700 & 0.45549400 \\
0.67926200 & -0.47110000 \\
0.41615800 & -1.91225500 \\
1.41554700 & -2.79642900 \\
-0.61481800 & -2.24120300 \\
3.14658600 & -1.09345000 \\
2.80079600 & -2.37955100 \\
1.18184200 & -3.85085400 \\
4.16276200 & -0.74387200 \\
3.54268400 & -3.12625500 \\
2.15088000 & -0.05945800 \\
2.29447000 & 0.07048700 \\
2.48996000 & 1.17099500 \\
2.28536200 & 2.22775000 \\
1.55993600 & 3.25690500 \\
0.58066600 & 2.89554600 \\
1.45312300 & 4.03127300 \\
2.12516900 & 3.65754400
\end{array}
$$

$-0.01661400$

$-1.14498600$

$-1.14066400$

$-0.00926700$

1.12642400

1.11877900

$-0.01985500$

$-2.03281200$

$-2.00887600$

2.01601300

2.00692300

$-0.00202700$

0.09118800

0.04703500

$-0.15618300$

0.06126200

$-0.17924300$

$-0.31858700$

$-0.26190500$

$-0.31758800$

$-0.57775200$

0.26488600

1.35040700

$-0.35350200$

0.63670500

$-0.01804600$

$-0.33565300$

0.74599300

$-0.86592600$

CBS-QB3 Enthalpy= -592.145207

CBS-QB3 Free Energy= -592.195346

$$
02
$$

$\mathrm{C}$
$\mathrm{C}$
$\mathrm{C}$
$\mathrm{C}$
3.77863800
3.61571300
2.36548500
1. 25899000

-0.88155400
0.36997200
0.97265700
0.31450200
$-0.11877800$
0.47597900
0.52055400
$-0.02830900$




$\begin{array}{lrrr}\mathrm{C} & 1.41776100 & -0.93358100 & -0.64219300 \\ \mathrm{C} & 2.67704800 & -1.52404900 & -0.68072500 \\ \mathrm{H} & 4.75655200 & -1.34733900 & -0.15167000 \\ \mathrm{H} & 4.46840100 & 0.88059000 & 0.90878200 \\ \mathrm{H} & 2.22085900 & 1.94942500 & 0.96098500 \\ \mathrm{H} & 0.57271500 & -1.42499400 & -1.10560900 \\ \mathrm{H} & 2.79709000 & -2.48642400 & -1.16502300 \\ \mathrm{C} & -1.25896800 & 0.31453600 & 0.02840900 \\ \mathrm{C} & -1.41791800 & -0.93341200 & 0.64253800 \\ \mathrm{C} & -2.36530600 & 0.97257700 & -0.52087400 \\ \mathrm{C} & -2.67721900 & -1.52383200 & 0.68092900 \\ \mathrm{H} & -0.57298200 & -1.42470100 & 1.10629200 \\ \mathrm{C} & -3.61556100 & 0.36991700 & -0.47648900 \\ \mathrm{H} & -2.22057900 & 1.94925300 & -0.96148500 \\ \mathrm{C} & -3.77866100 & -0.88144800 & 0.11853900 \\ \mathrm{H} & -2.79742100 & -2.48607400 & 1.16545200 \\ \mathrm{H} & -4.46812400 & 0.88047000 & -0.90961500 \\ \mathrm{H} & -4.75658900 & -1.34721000 & 0.15132400 \\ \mathrm{~N} & 0.00001100 & 0.98638500 & 0.00015600 \\ \mathrm{O} & -0.00000200 & 2.26682500 & 0.00024800\end{array}$

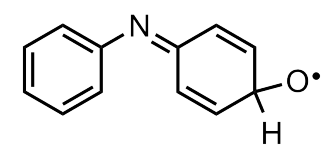

\section{CBS-QB3 Enthalpy= -592.104316 CBS-QB3 Free Energy= -592.155419}

$\begin{array}{lrrr}02 & & \\ \mathrm{C} & -4.14703000 & -0.69538900 & -0.13471700 \\ \mathrm{C} & -3.83428600 & 0.46585100 & -0.84260800 \\ \mathrm{C} & -2.59309700 & 1.07099100 & -0.69054800 \\ \mathrm{C} & -1.62094900 & 0.49979500 & 0.14604400 \\ \mathrm{C} & -1.94550100 & -0.66277900 & 0.86490100 \\ \mathrm{C} & -3.20140400 & -1.24724000 & 0.72640200 \\ \mathrm{H} & -5.12236900 & -1.15542700 & -0.24250000 \\ \mathrm{H} & -4.56754300 & 0.91015500 & -1.50658600 \\ \mathrm{H} & -2.34949000 & 1.98664600 & -1.21604300 \\ \mathrm{H} & -1.22030400 & -1.08211500 & 1.55281300 \\ \mathrm{H} & -3.44074500 & -2.13807200 & 1.29684600 \\ \mathrm{C} & 0.74384600 & 0.61676500 & 0.16574900 \\ \mathrm{C} & 0.99058400 & -0.74297700 & -0.34612100 \\ \mathrm{C} & 1.93085200 & 1.42774600 & 0.47228300 \\ \mathrm{C} & 2.22420800 & -1.25520500 & -0.41355900 \\ \mathrm{H} & 0.14194400 & -1.30646500 & -0.71386600 \\ \mathrm{C} & 3.17032800 & 0.93047700 & 0.43026100 \\ \mathrm{H} & 1.73731900 & 2.46030200 & 0.73935000 \\ \mathrm{H} & 2.41296300 & -2.23677800 & -0.83529600 \\ \mathrm{H} & 4.04110500 & 1.53612200 & 0.65557900 \\ \mathrm{C} & 3.44355600 & -0.50861300 & 0.06867900 \\ \mathrm{H} & 3.77140500 & -1.01664400 & 1.01571300 \\ \mathrm{~N} & -0.40665700 & 1.17861800 & 0.31348200 \\ \mathrm{O} & 4.55945800 & -0.70057200 & -0.70262300\end{array}$


<smiles>OC1C=CC=CC1=Nc1ccccc1</smiles>

CBS-QB3 Enthalpy $=-592.097722$
CBS-QB3 Free Energy $=-592.149527$

$$
\begin{gathered}
\mathrm{O}^{2} \\
\mathrm{C} \\
\mathrm{C} \\
\mathrm{C} \\
\mathrm{C} \\
\mathrm{C} \\
\mathrm{C} \\
\mathrm{H} \\
\mathrm{H} \\
\mathrm{H} \\
\mathrm{H} \\
\mathrm{H} \\
\mathrm{N} \\
\mathrm{C} \\
\mathrm{C} \\
\mathrm{C} \\
\mathrm{H} \\
\mathrm{C} \\
\mathrm{C} \\
\mathrm{H} \\
\mathrm{H} \\
\mathrm{H} \\
\mathrm{C} \\
\mathrm{H} \\
\mathrm{O}
\end{gathered}
$$

$$
\begin{aligned}
& -4.05599300 \\
& -3.58924900 \\
& -2.27229300 \\
& -1.38476400 \\
& -1.85961600 \\
& -3.18783400 \\
& -5.08818700 \\
& -4.25944300 \\
& -1.90626100 \\
& -1.19021800 \\
& -3.54334700 \\
& -0.08154900 \\
& 0.97174200 \\
& 1.03998900 \\
& 2.21454600 \\
& 0.12024000 \\
& 3.54573900 \\
& 3.47954900 \\
& 2.23583500 \\
& 4.48525100 \\
& 4.37973200 \\
& 2.31453100 \\
& 2.22318700 \\
& 2.47699500
\end{aligned}
$$<smiles>COc1ccc(Nc2ccccc2)cc1</smiles>

CBS-QB3 Enthalpy= -707.079181 CBS-QB3 Free Energy= -707.139365

$$
\begin{array}{r}
-0.41015700 \\
0.76301500 \\
1.16887300 \\
0.38237200 \\
-0.79274200 \\
-1.17760300 \\
-0.71460500 \\
1.37301400 \\
2.09088200 \\
-1.38218300 \\
-2.08290400 \\
0.86052700 \\
0.20019200 \\
-1.09522200 \\
-1.74008700 \\
-1.51284900 \\
0.02889500 \\
-1.18959300 \\
-2.69553200 \\
0.47755800 \\
-1.77959200 \\
0.89399300 \\
1.18408400 \\
2.10335300
\end{array}
$$

$-0.11826800$

$-0.71223900$

$-0.53370900$

0.21563800

0.81831700

0.65449000

$-0.24636300$

$-1.30783500$

$-0.96924100$

1.43429700

1.13441300

0.42393400

0.12609800

$-0.54540800$

$-0.70172400$

$-0.93575600$

0.31936600

$-0.23103500$

$-1.21553500$

0.62028500

$-0.36581300$

0.47018300

1.53941100

$-0.15320800$

\footnotetext{
01

$\mathrm{C}$
$\mathrm{C}$
$\mathrm{C}$
$\mathrm{C}$
$\mathrm{C}$
$\mathrm{C}$
$\mathrm{H}$
$\mathrm{H}$
$\mathrm{H}$
$\mathrm{H}$
$\mathrm{C}$
$\mathrm{C}$
$\mathrm{C}$
$\mathrm{C}$
}

$$
\begin{array}{r}
-0.24656800 \\
0.98786200 \\
1.40820000 \\
0.62188100 \\
-0.60845300 \\
-1.03551400 \\
1.60759300 \\
2.36379100 \\
-1.21766100 \\
-1.97669900 \\
0.42665100 \\
-0.88706500 \\
1.08738200 \\
-1.51306300
\end{array}
$$

$$
\begin{array}{r}
-0.17966100 \\
0.38518800 \\
0.41867500 \\
-0.11116000 \\
-0.69523300 \\
-0.73276600 \\
0.78958700 \\
0.87327400 \\
-1.15457900 \\
-1.19997300 \\
0.00360500 \\
0.49131400 \\
-0.35908800 \\
0.59381800
\end{array}
$$




$\begin{array}{lrrr}\mathrm{H} & -1.82608800 & -1.40745000 & 0.81063500 \\ \mathrm{C} & -5.04702600 & 0.45585300 & -0.23805800 \\ \mathrm{H} & -3.76236100 & 2.10134000 & -0.74355000 \\ \mathrm{C} & -5.13034000 & -0.85419300 & 0.23186100 \\ \mathrm{H} & -4.00245000 & -2.52747700 & 0.97531300 \\ \mathrm{H} & -5.94693200 & 0.98997200 & -0.52300600 \\ \mathrm{H} & -6.09010600 & -1.34948700 & 0.31643900 \\ \mathrm{~N} & -1.42288500 & 1.12051900 & -0.10099900 \\ \mathrm{H} & -1.50362300 & 2.12451700 & -0.11391600 \\ \mathrm{O} & 3.81795800 & -0.79748900 & -0.20091800 \\ \mathrm{O} & 4.82151900 & 0.26728800 & -0.14395500 \\ \mathrm{C} & 5.95333000 & -0.33260600 & 0.46093500 \\ \mathrm{H} & 6.31192800 & -1.18228600 & -0.12800600 \\ \mathrm{H} & 6.70655000 & 0.45818900 & 0.46069100 \\ \mathrm{H} & 5.73962000 & -0.64857000 & 1.48648700\end{array}$<smiles>[O-]c1ccc(Nc2ccccc2)cc1</smiles>

\section{CBS-QB3 Enthalpy= -592.189979 CBS-QB3 Free Energy= -592.240774}

$\begin{array}{lrrr}02 & & \\ \mathrm{C} & 3.56363800 & -0.38899000 & -0.09717200 \\ \mathrm{C} & 3.24082900 & 0.95497600 & 0.35439900 \\ \mathrm{C} & 1.95109300 & 1.40074900 & 0.41724500 \\ \mathrm{C} & 0.86331300 & 0.56381100 & 0.04262100 \\ \mathrm{C} & 1.13913100 & -0.74694200 & -0.42875800 \\ \mathrm{C} & 2.42812200 & -1.20429300 & -0.49387400 \\ \mathrm{H} & 4.07091700 & 1.58689900 & 0.64760400 \\ \mathrm{H} & 1.73637000 & 2.40541800 & 0.77150300 \\ \mathrm{H} & 0.32423700 & -1.37338500 & -0.76694700 \\ \mathrm{H} & 2.64913100 & -2.19744200 & -0.86732100 \\ \mathrm{C} & -1.65686900 & 0.43749800 & 0.06716500 \\ \mathrm{C} & -1.85862600 & -0.83919000 & 0.60926600 \\ \mathrm{C} & -2.74538000 & 1.12367100 & -0.49003500 \\ \mathrm{C} & -3.12250700 & -1.41907500 & 0.56551500 \\ \mathrm{H} & -1.03914100 & -1.36056800 & 1.08585200 \\ \mathrm{C} & -4.00656500 & 0.54090900 & -0.51297800 \\ \mathrm{H} & -2.59235500 & 2.11031200 & -0.91498300 \\ \mathrm{C} & -4.20159300 & -0.73806300 & 0.00577500 \\ \mathrm{H} & -3.26536800 & -2.40647500 & 0.98964000 \\ \mathrm{H} & -4.83726700 & 1.08509200 & -0.94748500 \\ \mathrm{H} & -5.18340800 & -1.19525700 & -0.01970400 \\ \mathrm{~N} & -0.40962900 & 1.08775900 & 0.11306600 \\ \mathrm{H} & -0.45630900 & 2.09240300 & 0.20000600 \\ \mathrm{O} & 4.73663600 & -0.80895700 & -0.15058000\end{array}$<smiles>O=C1C=CC(=Nc2ccccc2)C=C1</smiles>

CBS-QB3 Enthalpy= -591.594964 


\section{CBS-QB3 Free Energy= -591.644566}

$$
\begin{array}{ll}
0 & 1
\end{array}
$$

$$
\begin{array}{r}
-4.11626100 \\
-3.86124300 \\
-2.61813100 \\
-1.58489400 \\
-1.85273100 \\
-3.11290900 \\
-5.09452000 \\
-4.64165300 \\
-2.41487100 \\
-1.08596300 \\
-3.31134600 \\
0.78684200 \\
1.04425900 \\
1.95481800 \\
2.29721000 \\
0.19804800 \\
3.20773500 \\
1.73674500 \\
2.50812000 \\
4.07354700 \\
3.47847900 \\
-0.37205800 \\
4.61215700
\end{array}
$$

$$
\begin{array}{r}
-0.71107900 \\
0.51251800 \\
1.11749500 \\
0.47999900 \\
-0.74520100 \\
-1.32607400 \\
-1.17032300 \\
1.00481700 \\
2.08024400 \\
-1.20959200 \\
-2.26228600 \\
0.61149700 \\
-0.73771800 \\
1.43617300 \\
-1.21028900 \\
-1.32753400 \\
0.96066300 \\
2.44051500 \\
-2.19236800 \\
1.55508200 \\
-0.41229800 \\
1.16036800 \\
-0.86190400
\end{array}
$$
$-0.07363600$
$-0.69534700$
$-0.57097800$
0.13701800
0.77389400
0.67202000
$-0.15412100$
$-1.26449100$
$-1.02395700$
1.38250300
1.18167200
0.09691500
$-0.42696700$
0.42508600
$-0.53665100$
$-0.75599500$
0.34010200
0.76953100
$-0.94506400$
0.60806200
$-0.13390200$
0.26628700
$-0.21343600$<smiles>COc1ccccc1Nc1ccccc1</smiles>

CBS-QB3 Enthalpy= -707.082195 CBS-QB3 Free Energy= -707.141520

$$
\begin{array}{r}
2.87949200 \\
3.07432300 \\
1.98413000 \\
0.65309100 \\
0.47479900 \\
1.57458800 \\
3.72574300 \\
4.06697500 \\
-0.52152100 \\
1.40543600 \\
-1.73825900 \\
-2.28604000 \\
-2.60282300 \\
-3.66270700 \\
-1.63752800 \\
-3.97332900 \\
-2.18748200 \\
-4.51646300 \\
-4.06683800 \\
-4.62105500
\end{array}
$$

$$
\begin{array}{r}
-0.03191700 \\
-0.18726900 \\
-0.31519600 \\
-0.25873200 \\
-0.11895700 \\
-0.00356400 \\
0.05914500 \\
-0.23092000 \\
-0.12818200 \\
0.09813700 \\
-0.17468500 \\
0.77872800 \\
-0.89313900 \\
0.98190500 \\
1.38312000 \\
-0.66981900 \\
-1.63494800 \\
0.26248600 \\
1.72548700 \\
-1.23597400
\end{array}
$$




$\begin{array}{rrrr}\mathrm{H} & -5.58651800 & 0.08034600 & 0.42908000 \\ \mathrm{~N} & -0.36214200 & 0.30556700 & -0.40964100 \\ \mathrm{H} & -0.03359400 & 1.22083600 & -0.68277500 \\ \mathrm{O} & 2.18696800 & 1.19927500 & -0.57121100 \\ \mathrm{O} & 2.05188500 & 1.96354700 & 0.74567100 \\ \mathrm{C} & 2.92011700 & 3.06667600 & 0.60966600 \\ \mathrm{H} & 2.78538500 & 3.63193400 & 1.53643300 \\ \mathrm{H} & 3.96564700 & 2.75349900 & 0.51818700 \\ \mathrm{H} & 2.64402300 & 3.69814200 & -0.24202000\end{array}$<smiles>[O-]c1ccccc1Nc1ccccc1</smiles>

\section{CBS-QB3 Enthalpy= -592.198325 \\ CBS-QB3 Free Energy= $\mathbf{- 5 9 2 . 2 4 8 5 9 3}$}

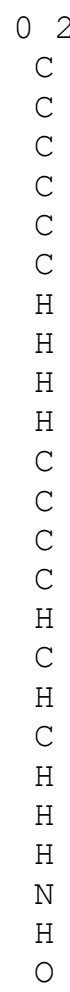<smiles>O=C1C=CC=CC1=Nc1ccccc1</smiles>

$$
\begin{array}{r}
3.72587600 \\
3.57156500 \\
2.26286700 \\
1.10304000 \\
1.30746300 \\
2.59619700 \\
4.71782200 \\
4.41605500 \\
0.46921700 \\
2.74473700 \\
-1.40974600 \\
-1.79752400 \\
-2.39489600 \\
-3.14016600 \\
-1.06270300 \\
-3.73194300 \\
-2.09846100 \\
-4.11351600 \\
-3.42612000 \\
-4.47869300 \\
-5.15674500 \\
-0.08275400 \\
0.07433900 \\
2.06306700
\end{array}
$$

$$
\begin{array}{r}
-0.13990500 \\
0.11049200 \\
0.16752800 \\
-0.03249100 \\
-0.31048600 \\
-0.36267000 \\
-0.18177100 \\
0.26743600 \\
-0.51621300 \\
-0.58973200 \\
0.02662400 \\
0.50064300 \\
-0.43302700 \\
0.48356900 \\
0.91787200 \\
-0.43701400 \\
-0.79532900 \\
0.01271700 \\
0.85791700 \\
-0.79819300 \\
0.00453600 \\
0.03544800 \\
0.12729100 \\
0.36777100
\end{array}
$$

CBS-QB3 Enthalpy $=-591.582347$ CBS-QB3 Free Energy= -591.632393

$$
\begin{array}{rr}
-4.02096800 & -0.45059400 \\
-3.61983800 & 0.65048400 \\
-2.30539600 & 1.09349800 \\
-1.34911400 & 0.40296400
\end{array}
$$

0.01356000
-0.74524800
-0.69948000
0.06570800 


$\mathrm{C}$
$\mathrm{C}$
$\mathrm{H}$
$\mathrm{H}$
$\mathrm{H}$
$\mathrm{H}$
$\mathrm{H}$
$\mathrm{N}$
$\mathrm{C}$
$\mathrm{C}$
$\mathrm{C}$
$\mathrm{H}$
$\mathrm{C}$
$\mathrm{C}$
$\mathrm{H}$
$\mathrm{H}$
$\mathrm{H}$
$\mathrm{C}$
$\mathrm{O}$

-1.76218600
-3.09104800
-5.05370100
-4.34137600
-1.98597700
-1.04791600
-3.40102400
-0.05689500
1.01342100
1.08687900
2.27700900
0.16626000
3.56666100
3.53057700
2.31641100
4.49476700
4.45251600
2.33279900
2.35569100

$-0.69803500$

$-1.10873800$

$-0.77857900$

1.17834000

1.96658700

$-1.19322600$

$-1.94609300$

0.92311700

0.21900100

$-1.19158500$

$-1.81653200$

$-1.70954300$

0.17387900

$-1.13114700$

$-2.85198700$

0.70359500

$-1.69543300$

0.96922900

2.14874700
0.83827000

0.81588200

$-0.00491700$

$-1.35856400$

$-1.25516700$

1. 48519100

1. 43114500

0.10426000

$-0.01715700$

$-0.39688200$

$-0.51278300$

$-0.63290000$

0.08710000

$-0.24585000$

$-0.83290100$

0.26605700

$-0.34198700$

0.21241200

0.50263000<smiles>Cc1ccc(Nc2ccc(C)cc2)cc1</smiles>

\section{CBS-QB3 Enthalpy= -595.492338 CBS-QB3 Free Energy= -595.550082}

O 2
$C$
$C$
$C$
$C$
$C$
$C$
$C$
$H$
$H$
$H$
$H$
N
C
C
C
C
H
C
H
C
H
H
C
H
H
H
C
H

$$
\begin{array}{r}
3.82493800 \\
3.59098700 \\
2.32494900 \\
1.20147400 \\
1.44725700 \\
2.73052600 \\
4.42484700 \\
2.14650000 \\
0.63207500 \\
2.89243900 \\
0.00000400 \\
-1.20147600 \\
-1.44727500 \\
-2.32493300 \\
-2.73054500 \\
-0.63210400 \\
-3.59097500 \\
-2.14647500 \\
-3.82494200 \\
-2.89247300 \\
-4.42482300 \\
-5.21210600 \\
-5.94152700 \\
-5.54123300 \\
-5.25465800 \\
5.21211300 \\
5.94141200
\end{array}
$$

-0.43325100
0.85674600
1.40758800
0.67625900
-0.61722900
-1.14234700
1.43587100
2.40929800
-1.17649300
-2.12471100
1.32184800
0.67626500
-0.61720400
1.40758500
-1.14231700
-1.17645300
0.85674600
2.40928700
-0.43323500
-2.12466500
1.43586500
-1.02219100
-0.32501600
-1.25174000
-1.94743800
-1.02218200
-0.32519600

$-0.05623900$

0.45139200

0.45215000

$-0.01672200$

$-0.54733500$

$-0.56796300$

0.83609700

0.82412500

$-0.98810800$

$-1.00063400$

0.00002200

0.01674900

0.54739700

$-0.45216800$

0.56801100

0.98821100

$-0.45142500$

$-0.82416000$

0.05623100

1.00071500

$-0.83616400$

0.05437900

0.47746600

$-0.96519100$

0.63229100

$-0.05444900$

$-0.47806400$ 


$\begin{array}{lrrr}\mathrm{H} & 5.54150900 & -1.25122500 & 0.96514600 \\ \mathrm{H} & 5.25453700 & -1.94770300 & -0.63193100\end{array}$<smiles>CON(c1ccc(C)cc1)c1ccc(C)cc1</smiles>

\section{CBS-QB3 Enthalpy= -785.497342 \\ CBS-QB3 Free Energy= -785.566668}

$$
\begin{aligned}
& \text { O C } \\
& \text { C } \\
& \text { C } \\
& \text { C } \\
& \text { C } \\
& \text { C } \\
& \text { C } \\
& \text { H } \\
& \text { H } \\
& \text { H } \\
& \text { H } \\
& \text { C } \\
& \text { C } \\
& \text { C } \\
& \text { C } \\
& \text { H } \\
& \text { C } \\
& \text { H } \\
& \text { C } \\
& \text { H } \\
& \text { H } \\
& \text { N } \\
& \text { O } \\
& \text { O } \\
& \text { C } \\
& \text { H } \\
& \text { H } \\
& \text { H } \\
& \text { C } \\
& \text { H } \\
& \text { H } \\
& \text { H } \\
& \text { C } \\
& \text { H } \\
& \text { H } \\
& \text { H }
\end{aligned}
$$<smiles>Cc1ccc(N([O-])c2ccc(C)cc2)cc1</smiles>

CBS-QB3 Enthalpy= -670.604195 CBS-QB3 Free Energy= -670.663982

$$
\begin{array}{r}
-3.71950100 \\
-3.60511000 \\
-2.38303700 \\
-1.23197000 \\
-1.32635800 \\
-2.55940100 \\
-4.48989500 \\
-2.30661400 \\
-0.44828300 \\
-2.61777500 \\
1.28971400 \\
1.55397700 \\
2.30025400 \\
2.81915200 \\
0.77959000 \\
3.55922700 \\
2.07368200 \\
3.84586200 \\
3.01162500 \\
4.33482500 \\
0.00997500 \\
-0.02449400 \\
-0.10202400 \\
-0.44673800 \\
-0.44756300 \\
0.28445600 \\
-1.44838700 \\
5.22580800 \\
5.90043600 \\
5.66984400 \\
5.20233000 \\
-5.04867300 \\
-5.86550700 \\
-5.27311000 \\
-5.05658700
\end{array}
$$

0.20892100

$-0.67313800$

$-0.91944800$

$-0.27258500$

0.62258000

0.84998100

$-1.18057700$

$-1.59919400$

1.15258600

1.55501400

$-0.29875900$

$-0.72894600$

0.30502600

$-0.53845300$

$-1.21721600$

0.47944900

0.64303900

0.06557000

$-0.87765800$

0.95770300

$-0.53546700$

$-0.74275400$

0.75122800

0.37924500

1.30544400

$-0.30774200$

$-0.06929900$

0.24179600

$-0.55471200$

1.19219500

0.21682800

0.44087100

0.46555800

$-0.35850600$

1. 38434900

02 


$\begin{array}{lrrr}\mathrm{C} & 3.61238900 & 0.60418200 & 0.50423300 \\ \mathrm{C} & 2.36333700 & 1.20508500 & 0.53646300 \\ \mathrm{C} & 1.25801700 & 0.54483900 & -0.01282300 \\ \mathrm{C} & 1.43076000 & -0.70578200 & -0.61398800 \\ \mathrm{C} & 2.69270100 & -1.29122100 & -0.63707700 \\ \mathrm{H} & 4.45916600 & 1.12656000 & 0.93791000 \\ \mathrm{H} & 2.21888900 & 2.18677600 & 0.96613300 \\ \mathrm{H} & 0.59486700 & -1.20618200 & -1.08455100 \\ \mathrm{H} & 2.81435800 & -2.25685300 & -1.11679400 \\ \mathrm{C} & -1.25799900 & 0.54482200 & 0.01297900 \\ \mathrm{C} & -1.43091400 & -0.7055690 & 0.61452800 \\ \mathrm{C} & -2.36313600 & 1.20488200 & -0.53689300 \\ \mathrm{C} & -2.69287200 & -1.29101000 & 0.63743200 \\ \mathrm{H} & -0.59514600 & -1.20580300 & 1.08549500 \\ \mathrm{C} & -3.61219400 & 0.60399600 & -0.50484600 \\ \mathrm{H} & -2.21855300 & 2.18642000 & -0.96687200 \\ \mathrm{C} & -3.80435600 & -0.65735500 & 0.07487200 \\ \mathrm{H} & -2.81467900 & -2.25648000 & 1.11743400 \\ \mathrm{H} & -4.45883100 & 1.12620800 & -0.93899900 \\ \mathrm{~N} & 0.00002000 & 1.21637700 & 0.00017800 \\ \mathrm{O} & -0.00002400 & 2.49769300 & 0.00028300 \\ \mathrm{C} & -5.16489500 & -1.30913000 & 0.07932800 \\ \mathrm{H} & -5.94982700 & -0.59086500 & 0.33048700 \\ \mathrm{H} & -5.40726900 & -1.72286600 & -0.90571700 \\ \mathrm{H} & -5.21246400 & -2.12749700 & 0.80067100 \\ \mathrm{C} & 5.16483600 & -1.30930200 & -0.07956300 \\ \mathrm{H} & 5.95012200 & -0.59059800 & -0.32829500 \\ \mathrm{H} & 5.40610100 & -1.72528500 & 0.90481500 \\ \mathrm{H} & 5.21304700 & -2.12609700 & -0.80265200\end{array}$

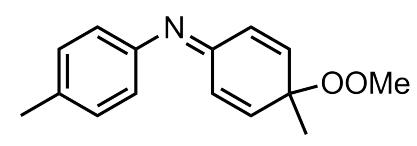

\section{CBS-QB3 Enthalpy $=-785.501909$ CBS-QB3 Free Energy= -785.568445}

$\mathrm{O}^{\mathrm{C}}{ }^{\mathrm{C}}$
$\mathrm{C}$
$\mathrm{C}$
$\mathrm{C}$
$\mathrm{C}$
$\mathrm{C}$
$\mathrm{H}$
$\mathrm{H}$
$\mathrm{H}$
$\mathrm{H}$
$\mathrm{C}$
$\mathrm{C}$
$\mathrm{C}$
$\mathrm{C}$
$\mathrm{H}$
$\mathrm{C}$
$\mathrm{H}$
$\mathrm{H}$

$$
\begin{array}{r}
4.85036000 \\
4.43643200 \\
3.17412400 \\
2.25235600 \\
2.66309900 \\
3.94321700 \\
5.12217900 \\
2.87239800 \\
1.98672400 \\
4.24033600 \\
-0.11408700 \\
-0.32380000 \\
-1.32526400 \\
-1.53638600 \\
0.53114500 \\
-2.53504000 \\
-1.17378400 \\
-1.68634000
\end{array}
$$

$$
\begin{array}{r}
-0.34258000 \\
-0.51604000 \\
-0.12480200 \\
0.41930300 \\
0.60580900 \\
0.23408100 \\
-0.95337900 \\
-0.24056800 \\
1.07270600 \\
0.39866800 \\
0.53879400 \\
-0.43550400 \\
1.15840200 \\
-0.66160100 \\
-1.00177600 \\
0.96278400 \\
1.80242700 \\
-1.41861600
\end{array}
$$




$\begin{array}{lrrr}\mathrm{H} & -3.41331600 & -0.98221100 & 1.44549700 \\ \mathrm{C} & -2.77031100 & 0.64471000 & 0.10085200 \\ \mathrm{~N} & 1.01330600 & -1.09093500 & 0.85307300 \\ \mathrm{O} & -3.70603000 & 0.34158300 & -0.97085100 \\ \mathrm{O} & -4.99714700 & 0.00434600 & -0.37189300 \\ \mathrm{C} & -5.44398500 & -1.14849900 & -1.06484100 \\ \mathrm{H} & -6.43139500 & -1.35494200 & -0.64467700 \\ \mathrm{H} & -5.53555900 & -0.95214300 & -2.13735700 \\ \mathrm{H} & -4.77781300 & -2.00082500 & -0.89782800 \\ \mathrm{C} & -3.30137400 & 1.82532000 & 0.93528300 \\ \mathrm{H} & -3.48172800 & 2.68529100 & 0.28611800 \\ \mathrm{H} & -4.24025500 & 1.55067700 & 1.41706100 \\ \mathrm{H} & -2.57500700 & 2.10221900 & 1.70189700 \\ \mathrm{C} & 6.24152300 & 0.67292200 & -0.73492000 \\ \mathrm{H} & 6.99223500 & 0.27729200 & -0.04216300 \\ \mathrm{H} & 6.50423200 & 0.31479700 & -1.73443400 \\ \mathrm{H} & 6.33304600 & 1.76111800 & -0.73326500\end{array}$

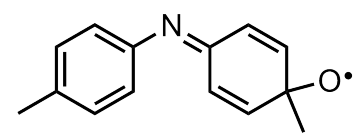

\section{CBS-QB3 Enthalpy $=-670.565816$ CBS-QB3 Free Energy= -670.625414}

$\begin{array}{lrrr}\mathrm{O}^{2} & & \\ \mathrm{C} & 4.07374500 & -0.43195200 & 0.06617700 \\ \mathrm{C} & 3.76110700 & 0.82961700 & 0.58950100 \\ \mathrm{C} & 2.50345100 & 1.39134900 & 0.42254300 \\ \mathrm{C} & 1.48753900 & 0.68619300 & -0.24195100 \\ \mathrm{C} & 1.79576600 & -0.57358800 & -0.77760700 \\ \mathrm{C} & 3.07018300 & -1.11319300 & -0.62652700 \\ \mathrm{H} & 4.52227300 & 1.38718700 & 1.12661200 \\ \mathrm{H} & 2.27910400 & 2.37791900 & 0.81060900 \\ \mathrm{H} & 1.04199800 & -1.11337200 & -1.33925400 \\ \mathrm{H} & 3.28605000 & -2.08574500 & -1.05805600 \\ \mathrm{C} & -0.87933700 & 0.76984800 & -0.15783400 \\ \mathrm{C} & -1.08315800 & -0.51200900 & 0.53823200 \\ \mathrm{C} & -2.09286500 & 1.51769500 & -0.51461700 \\ \mathrm{C} & -2.30575500 & -1.01896800 & 0.73023100 \\ \mathrm{H} & -0.21147700 & -1.01861200 & 0.93354800 \\ \mathrm{C} & -3.31956000 & 1.01577100 & -0.34860000 \\ \mathrm{H} & -1.93015300 & 2.50360300 & -0.93475400 \\ \mathrm{H} & -2.45458100 & -1.93544100 & 1.29278500 \\ \mathrm{H} & -4.20701900 & 1.58121700 & -0.61331800 \\ \mathrm{C} & -3.57075400 & -0.35736700 & 0.23265200 \\ \mathrm{~N} & 0.25329000 & 1.32088400 & -0.43390700 \\ \mathrm{O} & -4.53373500 & -0.31815000 & 1.22183500 \\ \mathrm{C} & -4.25121600 & -1.27211300 & -0.85569200 \\ \mathrm{H} & -4.46503300 & -2.25429800 & -0.43274500 \\ \mathrm{H} & -5.18042800 & -0.81194500 & -1.19325600 \\ \mathrm{H} & -3.55906200 & -1.36990200 & -1.69324900 \\ \mathrm{C} & 5.45992700 & -1.01126800 & 0.21287100 \\ \mathrm{H} & 6.15314900 & -0.57020300 & -0.51200400 \\ \mathrm{H} & 5.86823800 & -0.82013100 & 1.20884000 \\ \mathrm{H} & 5.45935500 & -2.09135900 & 0.05062700\end{array}$


<smiles>Cc1ccc(N=C2C=CC(=O)C=C2)cc1</smiles>

CBS-QB3 Enthalpy= -630.824031

CBS-QB3 Free Energy= -630.878811

\begin{tabular}{|c|c|c|c|}
\hline $\mathrm{C}$ & -373677600 & -036469900 & 001017500 \\
\hline $\mathrm{C}$ & -3.39712000 & 0.85135500 & -0.62167700 \\
\hline $\mathrm{C}$ & -2.11735300 & 1.37317300 & -0.52646300 \\
\hline $\mathrm{C}$ & -1.10308300 & 0.66452700 & 0.14230800 \\
\hline $\mathrm{C}$ & -1.44064900 & -0.54479900 & 0.77321600 \\
\hline $\mathrm{C}$ & -2.73963200 & -1.03771000 & 0.69997300 \\
\hline $\mathrm{H}$ & -4.15681000 & 1.40618700 & -1.16314600 \\
\hline $\mathrm{H}$ & -1.86837700 & 2.32743800 & -0.97480900 \\
\hline $\mathrm{H}$ & -0.69699500 & -1.06779400 & 1.36264000 \\
\hline $\mathrm{H}$ & -2.98228500 & -1.96292000 & 1.21245400 \\
\hline $\mathrm{N}$ & 0.15121300 & 1.26689100 & 0.23805700 \\
\hline $\mathrm{C}$ & 1.27298600 & 0.64031300 & 0.07793000 \\
\hline C & 1.44411100 & -0.73417200 & -0.41218600 \\
\hline C & 2.66496300 & -1.28740000 & -0.51206400 \\
\hline $\mathrm{H}$ & 0.56278100 & -1.27713300 & -0.72933400 \\
\hline $\mathrm{C}$ & 3.71222600 & 0.84256400 & 0.30558100 \\
\hline C & 3.89517200 & -0.55648500 & -0.13109500 \\
\hline $\mathrm{H}$ & 2.81214600 & -2.28961300 & -0.89904800 \\
\hline $\mathrm{H}$ & 4.61420600 & 1.38873100 & 0.55645100 \\
\hline C & -5.13779000 & -0.91421700 & -0.11195100 \\
\hline $\mathrm{H}$ & -5.88271000 & -0.15500000 & 0.14299600 \\
\hline $\mathrm{H}$ & -5.35582800 & -1.25098400 & -1.13104900 \\
\hline $\mathrm{H}$ & -5.28186800 & -1.76514200 & 0.55638100 \\
\hline $\mathrm{C}$ & 2.49142100 & 1.39824600 & 0.37863600 \\
\hline $\mathrm{H}$ & 2.33840500 & 2.42330600 & 0.69610800 \\
\hline 0 & 4.99849800 & -1.07868600 & -0.20080500 \\
\hline
\end{tabular}<smiles>COC1C=C(C)C=CC1=Nc1ccc(C)cc1</smiles>

CBS-QB3 Enthalpy= -785.493752

CBS-QB3 Free Energy= -785.561390

\footnotetext{
01

C

C

C

C

C

C

$\mathrm{H}$

$\mathrm{H}$

$\mathrm{H}$

$\mathrm{H}$
}

4.42828400
3.94150300
2.59209900
1.66582900
2.14529000
3.50585700
4.63597400
2.23048300
1.45088700
3.85464200

-0.13992900
0.25435700
0.50147300
0.32106200
-0.05909000
-0.27755200
0.38521500
0.83171900
-0.14621300
-0.55765300

$-0.13904300$

1.11467900

1.32236000

0.28341600

$-0.97800200$

$-1.17846600$

1.93903400

2.28912300

$-1.80577200$

$-2.16751800$ 


$\begin{array}{lrrr}\text { N } & 0.32105700 & 0.64138400 & 0.51606400 \\ \mathrm{C} & -0.64877000 & -0.16276700 & 0.26769600 \\ \mathrm{C} & -0.55247000 & -1.57243800 & -0.08966800 \\ \mathrm{C} & -1.65411200 & -2.34904500 & -0.13948800 \\ \mathrm{H} & 0.42958300 & -1.99816100 & -0.24745600 \\ \mathrm{C} & -3.18153200 & -0.54434600 & 0.38476800 \\ \mathrm{C} & -3.00769200 & -1.84454300 & 0.10256000 \\ \mathrm{H} & -1.54495800 & -3.40809000 & -0.35581900 \\ \mathrm{H} & -4.17045200 & -0.13306500 & 0.55367100 \\ \mathrm{C} & -4.14818100 & -2.82492700 & 0.02088100 \\ \mathrm{H} & -5.10441600 & -2.34038100 & 0.22319800 \\ \mathrm{H} & -4.20054800 & -3.28017800 & -0.97403700 \\ \mathrm{H} & -4.01892800 & -3.64113600 & 0.73995100 \\ \mathrm{C} & 5.90004100 & -0.40003200 & -0.35151100 \\ \mathrm{H} & 6.51069800 & 0.42087800 & 0.03563500 \\ \mathrm{H} & 6.22058800 & -1.31209200 & 0.16354600 \\ \mathrm{H} & 6.13349000 & -0.52001900 & -1.41139200 \\ \mathrm{C} & -2.04824600 & 0.43844000 & 0.41612800 \\ \mathrm{H} & -2.06389500 & 1.03326700 & 1.33447400 \\ \mathrm{O} & -2.16527400 & 1.35687700 & -0.69834800 \\ \mathrm{O} & -3.23383300 & 2.29087700 & -0.37281500 \\ \mathrm{C} & -2.60517900 & 3.55194200 & -0.19154300 \\ \mathrm{H} & -3.42297800 & 4.23532600 & 0.05053000 \\ \mathrm{H} & -1.88298200 & 3.52536200 & 0.63078500 \\ \mathrm{H} & -2.10805700 & 3.87787200 & -1.10969700\end{array}$<smiles>CC1=CC([O])C(=Nc2ccc(C)cc2)C=C1</smiles>

CBS-QB3 Enthalpy $=-670.557355$ CBS-QB3 Free Energy= -670.618127

$\mathrm{O}^{2}$
$\mathrm{C}$
$\mathrm{C}$
$\mathrm{C}$
$\mathrm{C}$
$\mathrm{C}$
$\mathrm{C}$
$\mathrm{H}$
$\mathrm{H}$
$\mathrm{H}$
$\mathrm{H}$
$\mathrm{N}$
$\mathrm{C}$
$\mathrm{C}$
$\mathrm{C}$
$\mathrm{H}$
$\mathrm{C}$
$\mathrm{C}$
$\mathrm{H}$
$\mathrm{H}$
$\mathrm{C}$
$\mathrm{H}$
$\mathrm{H}$

$$
\begin{array}{rr}
-4.00226200 & -0.39792600 \\
-3.57876700 & 0.40560800 \\
-2.28792800 & 0.91101700 \\
-1.35167800 & 0.59075800 \\
-1.76879400 & -0.20560200 \\
-3.07531800 & -0.68279800 \\
-4.27900100 & 0.65092900 \\
-1.97898500 & 1.55253600 \\
-1.07370700 & -0.41989100 \\
-3.37792900 & -1.28852900 \\
-0.07556800 & 1.16501800 \\
1.01146400 & 0.49177600 \\
1.15378500 & -0.95929100 \\
2.36805100 & -1.54270600 \\
0.25287300 & -1.55843700 \\
3.59898300 & 0.54134200 \\
3.62904800 & -0.79697900 \\
2.43051600 & -2.62409200 \\
4.50877800 & 1.12920100 \\
4.91294800 & -1.58372100 \\
5.78398700 & -0.92848400 \\
4.97081200 & -2.28623100
\end{array}
$$

$$
\begin{array}{r}
0.04299600 \\
-1.02410200 \\
-1.08226500 \\
-0.08711700 \\
0.98923100 \\
1.04811400 \\
-1.81677500 \\
-1.89919100 \\
1.79294900 \\
1.89664100 \\
-0.15144100 \\
-0.09508400 \\
-0.15152900 \\
-0.21015200 \\
-0.18596700 \\
-0.08506100 \\
-0.16937100 \\
-0.29276700 \\
-0.02757000 \\
-0.21613100 \\
-0.17174600 \\
0.62210000
\end{array}
$$




$\begin{array}{rrrr}\mathrm{H} & 4.97673400 & -2.17588600 & -1.13537700 \\ \mathrm{C} & -5.42360800 & -0.90074100 & 0.11766700 \\ \mathrm{H} & -6.11274400 & -0.10066300 & 0.40945400 \\ \mathrm{H} & -5.76300800 & -1.28298100 & -0.84908200 \\ \mathrm{H} & -5.52368100 & -1.70389400 & 0.85084900 \\ \mathrm{C} & 2.31107600 & 1.32513500 & -0.00277500 \\ \mathrm{H} & 2.27094300 & 2.00702700 & -0.88390400 \\ \mathrm{O} & 2.30767500 & 2.21238000 & 1.03799300\end{array}$<smiles>COc1cc(C)ccc1Nc1ccc(C)cc1</smiles>

CBS-QB3 Enthalpy= -785.540346 CBS-QB3 Free Energy= -785.609486

$\begin{array}{lrrr}\mathrm{O}^{1} & & & \\ \mathrm{C} & -4.43332400 & -0.26732300 & 0.18805000 \\ \mathrm{C} & -4.00455600 & 0.64503000 & -0.78210300 \\ \mathrm{C} & -2.65550900 & 0.87382300 & -1.01702500 \\ \mathrm{C} & -1.67266900 & 0.20616200 & -0.27074500 \\ \mathrm{C} & -2.09182300 & -0.68992500 & 0.72156700 \\ \mathrm{C} & -3.44810200 & -0.92088500 & 0.93197800 \\ \mathrm{H} & -4.73958500 & 1.18191100 & -1.37373500 \\ \mathrm{H} & -2.35048400 & 1.57385000 & -1.78806900 \\ \mathrm{H} & -1.36304300 & -1.17902500 & 1.35381000 \\ \mathrm{H} & -3.74334900 & -1.61657300 & 1.71143200 \\ \mathrm{~N} & -0.32741700 & 0.51384200 & -0.51467400 \\ \mathrm{C} & 0.80637200 & -0.25050900 & -0.32896300 \\ \mathrm{C} & 0.82697000 & -1.64296100 & -0.15302700 \\ \mathrm{C} & 2.03247700 & -2.32300900 & -0.01753600 \\ \mathrm{H} & -0.10006000 & -2.19928000 & -0.15591100 \\ \mathrm{C} & 3.25501100 & -0.28954700 & -0.24663000 \\ \mathrm{C} & 3.27041600 & -1.67177200 & -0.05685300 \\ \mathrm{H} & 2.00551300 & -3.40129700 & 0.10601300 \\ \mathrm{H} & 4.17870200 & 0.27431100 & -0.31799800 \\ \mathrm{C} & 4.56485600 & -2.42879600 & 0.11820900 \\ \mathrm{H} & 5.39892800 & -1.90573500 & -0.35539700 \\ \mathrm{H} & 4.81607700 & -2.55376400 & 1.17763600 \\ \mathrm{H} & 4.50412700 & -3.42800200 & -0.32072500 \\ \mathrm{C} & -5.90047000 & -0.54782100 & 0.40613900 \\ \mathrm{H} & -6.50565300 & 0.34950600 & 0.25376700 \\ \mathrm{H} & -6.26849100 & -1.31036500 & -0.28971300 \\ \mathrm{H} & -6.08960000 & -0.91237500 & 1.41860300 \\ \mathrm{C} & 2.05909200 & 0.40647600 & -0.38751300 \\ \mathrm{O} & 2.09021000 & 1.74900700 & -0.68053100 \\ \mathrm{O} & 1.70145200 & 2.52880100 & 0.55974700 \\ \mathrm{C} & 2.87304500 & 3.18959900 & 0.99216700 \\ \mathrm{H} & 2.54739000 & 3.77982100 & 1.85391500 \\ \mathrm{H} & 3.64643400 & 2.48215200 & 1.31159300 \\ \mathrm{H} & 3.26646900 & 3.85769500 & 0.21947300 \\ \mathrm{H} & -0.13547300 & 1.45655200 & -0.82199300 \\ & & & \end{array}$


<smiles>Cc1ccc(Nc2ccc(C)cc2[O-])cc1</smiles>

CBS-QB3 Enthalpy= -670.656798
CBS-QB3 Free Energy= -670.716321

$\begin{array}{lrrr}\mathrm{O}^{2} & & & \\ \mathrm{C} & 4.09351800 & -0.39117900 & -0.01131400 \\ \mathrm{C} & 3.72344600 & 0.87944100 & 0.44579500 \\ \mathrm{C} & 2.39913400 & 1.29158400 & 0.44309500 \\ \mathrm{C} & 1.38735100 & 0.43995400 & -0.02655600 \\ \mathrm{C} & 1.74787000 & -0.82376100 & -0.51233300 \\ \mathrm{C} & 3.08013900 & -1.22342200 & -0.49418800 \\ \mathrm{H} & 4.48563100 & 1.55812100 & 0.81471900 \\ \mathrm{H} & 2.13469200 & 2.27690900 & 0.81185200 \\ \mathrm{H} & 1.00090200 & -1.47540500 & -0.94366500 \\ \mathrm{H} & 3.33630800 & -2.20364700 & -0.88318200 \\ \mathrm{~N} & 0.07388600 & 0.92359600 & -0.03549600 \\ \mathrm{C} & -1.12973100 & 0.29735500 & 0.01319300 \\ \mathrm{C} & -1.37750500 & -1.06062800 & 0.26631300 \\ \mathrm{C} & -2.67832100 & -1.52615000 & 0.30238500 \\ \mathrm{H} & -0.56116400 & -1.74066600 & 0.46683200 \\ \mathrm{C} & -3.58702200 & 0.66312200 & -0.13630300 \\ \mathrm{C} & -3.80225600 & -0.67179000 & 0.08819900 \\ \mathrm{H} & -2.85395300 & -2.57611100 & 0.51219800 \\ \mathrm{H} & -4.40906200 & 1.35361300 & -0.28735300 \\ \mathrm{C} & -5.19082900 & -1.25837800 & 0.12594400 \\ \mathrm{H} & -5.95084500 & -0.48925600 & -0.01924100 \\ \mathrm{H} & -5.32010600 & -2.01411000 & -0.65593800 \\ \mathrm{H} & -5.38480000 & -1.75105600 & 1.08428900 \\ \mathrm{C} & 5.53085000 & -0.84778000 & 0.03166200 \\ \mathrm{H} & 6.21830100 & -0.01967500 & -0.15730100 \\ \mathrm{H} & 5.78415300 & -1.26365900 & 1.01330200 \\ \mathrm{H} & 5.72464700 & -1.62400600 & -0.71189900 \\ \mathrm{C} & -2.26421800 & 1.23235500 & -0.17634600 \\ \mathrm{O} & -2.03240100 & 2.44583700 & -0.35492800 \\ \mathrm{H} & -0.05725700 & 1.93273800 & -0.11400000\end{array}$<smiles>CC1=CC(=O)C(=Nc2ccc(C)cc2)C=C1</smiles>

CBS-QB3 Enthalpy= -670.043402 CBS-QB3 Free Energy= -670.101979

\footnotetext{
01

C

$\mathrm{C}$

C

C

C

C

$\mathrm{H}$

$\mathrm{H}$
}

$-3.98405300$

$-3.60682300$

$-2.31467300$

$-1.32397100$

$-1.69738700$

$-3.00960200$

$-4.34717900$

$-2.03581000$
-0.40661400
0.71263200
1.20970100
0.56310800
-0.55062500
-1.01273200
1.21437500
2.09465600

0.00661000

$-0.75042900$

$-0.70744300$

0.05394700

0.82503700

0.80332300

$-1.36569600$

$-1.26648300$ 


$\mathrm{H}$
$\mathrm{H}$
$\mathrm{N}$
$\mathrm{C}$
$\mathrm{C}$
$\mathrm{C}$
$\mathrm{H}$
$\mathrm{C}$
$\mathrm{C}$
$\mathrm{H}$
$\mathrm{H}$
$\mathrm{C}$
$\mathrm{H}$
$\mathrm{H}$
$\mathrm{H}$
$\mathrm{C}$
$\mathrm{H}$
$\mathrm{H}$
$\mathrm{H}$
$\mathrm{C}$
$\mathrm{O}$

-0.96861600
-3.28034600
-0.05700800
1.04611300
1.19213800
2.40950900
0.30272900
3.59248000
3.64561100
2.49547100
4.48919600
4.94073200
5.79670700
4.94898000
5.07057000
-5.40524000
-6.09833700
-5.72852300
-5.51550500
2.32895800
2.29837000

-1.01952200
-1.86160500
1.14003100
0.48167500
-0.93469800
-1.50880400
-1.50291200
0.54506300
-0.77553500
-2.55200900
1.12739400
-1.53512500
-0.89056400
-2.35738600
-1.98359100
-0.91174900
-0.16591600
-1.13378400
-1.82208200
1.29109800
2.48354600

1.47542500

1. 42298900

0.08499000

0.00203700

$-0.32809900$

$-0.39916700$

$-0.56881500$

0.15334100

$-0.12909400$

$-0.68769300$

0.33434200

$-0.20412800$

$-0.00272200$

0.51967200

$-1.19501300$

$-0.01965900$

0.38293100

$-1.04123000$

0.57263800

0.21979300

0.45853900<smiles>CN(C)c1ncc(Nc2ccccc2)cn1</smiles>

\section{CBS-QB3 Enthalpy= -682.848895 CBS-QB3 Free Energy= -682.907381}

$\mathrm{O}^{2}$
$\mathrm{C}$
$\mathrm{C}$
$\mathrm{C}$
$\mathrm{C}$
$\mathrm{C}$
$\mathrm{C}$
$\mathrm{H}$
$\mathrm{H}$
$\mathrm{H}$
$\mathrm{H}$
$\mathrm{H}$
$\mathrm{C}$
$\mathrm{C}$
$\mathrm{C}$
$\mathrm{H}$
$\mathrm{H}$
$\mathrm{C}$
$\mathrm{N}$
$\mathrm{N}$
$\mathrm{N}$
$\mathrm{N}$
$\mathrm{C}$
$\mathrm{H}$
$\mathrm{H}$
$\mathrm{H}$

$$
\begin{array}{rr}
4.88167000 & 0.94914800 \\
4.82096400 & -0.31187300 \\
3.63077100 & -1.02000600 \\
2.44261900 & -0.47109000 \\
2.52733800 & 0.80118900 \\
3.73240600 & 1.48891700 \\
5.81729800 & 1.49520900 \\
5.71279400 & -0.74606000 \\
3.56982400 & -2.00681900 \\
1.65606200 & 1.21565700 \\
3.77930700 & 2.45191100 \\
0.06668600 & -0.76509800 \\
-0.37125300 & 0.52492300 \\
-1.00463700 & -1.64755100 \\
0.34315400 & 1.25357400 \\
-0.76054800 & -2.67241700 \\
-2.55636500 & -0.01951200 \\
1.32098000 & -1.26268700 \\
-2.27195200 & -1.29824800 \\
-1.64042600 & 0.88835600 \\
-3.86182600 & 0.36937600 \\
-4.92361600 & -0.53204200 \\
-5.46298700 & -0.10395900 \\
-5.63878000 & -0.67944000 \\
-4.49678500 & -1.48900900
\end{array}
$$

0.10801600

$-0.49194400$

$-0.51540200$

0.02488100

0.64458400

0.68770300

0.14011800

$-0.93033000$

$-0.95808600$

1.13651800

1.18466700

$-0.03276500$

$-0.44564500$

0.28641500

$-0.81591900$

0.55449200

$-0.06119800$

$-0.01591000$

0.29146700

$-0.46700700$

$-0.02386300$

0.39513000

1. 24757100

$-0.42239500$

0.67725900 


$\begin{array}{lrrr}\mathrm{C} & -4.27657200 & 1.71095800 & -0.40209900 \\ \mathrm{H} & -3.41242500 & 2.27688700 & -0.73470300 \\ \mathrm{H} & -5.01523000 & 1.65978500 & -1.20987600 \\ \mathrm{H} & -4.73918100 & 2.21933900 & 0.45183300\end{array}$<smiles>CON(c1ccccc1)c1cnc(N(C)C)nc1</smiles>

\section{CBS-QB3 Enthalpy $=-872.849436$ CBS-QB3 Free Energy= -872.919476}

$\begin{array}{lrrr}\mathrm{O}^{1} & & & \\ \mathrm{C} & 4.28382000 & -2.19134000 & -0.41779200 \\ \mathrm{C} & 4.53414400 & -0.82538600 & -0.29291300 \\ \mathrm{C} & 3.51689500 & 0.05870200 & 0.04710900 \\ \mathrm{C} & 2.22383100 & -0.42915100 & 0.26536400 \\ \mathrm{C} & 1.96316500 & -1.79866300 & 0.14035100 \\ \mathrm{C} & 2.99412400 & -2.66927600 & -0.19996700 \\ \mathrm{H} & 5.08284200 & -2.87303200 & -0.68433800 \\ \mathrm{H} & 5.53212600 & -0.43908100 & -0.46772500 \\ \mathrm{H} & 3.69923200 & 1.12050800 & 0.12274600 \\ \mathrm{H} & 0.96495700 & -2.17971800 & 0.31326100 \\ \mathrm{H} & 2.78285300 & -3.72866600 & -0.29249200 \\ \mathrm{C} & -0.19303800 & 0.17282900 & 0.42031100 \\ \mathrm{C} & -1.08687600 & -0.01452300 & 1.47083500 \\ \mathrm{C} & -0.73796400 & 0.13470000 & -0.86515300 \\ \mathrm{H} & -0.73325600 & 0.01932200 & 2.49857700 \\ \mathrm{H} & -0.10506200 & 0.29821000 & -1.73231200 \\ \mathrm{C} & -2.81131500 & -0.26767400 & 0.00154000 \\ \mathrm{~N} & 1.18570900 & 0.45680000 & 0.66510200 \\ \mathrm{O} & 1.49066600 & 1.72234300 & 0.82911200 \\ \mathrm{O} & 1.54743800 & 2.45298100 & -0.63821800 \\ \mathrm{C} & 1.24821000 & 3.79153900 & -0.35390400 \\ \mathrm{H} & 1.32710700 & 4.31868900 & -1.31211500 \\ \mathrm{H} & 0.23185700 & 3.91926000 & 0.04010300 \\ \mathrm{H} & 1.96896500 & 4.23777800 & 0.34392700 \\ \mathrm{~N} & -4.13532100 & -0.50424900 & -0.21318200 \\ \mathrm{~N} & -2.38012000 & -0.23339300 & 1.28401800 \\ \mathrm{~N} & -2.02449300 & -0.08340200 & -1.08597900 \\ \mathrm{C} & -4.70583700 & -0.53967700 & -1.55055100 \\ \mathrm{H} & -5.19936700 & -1.50284400 & -1.72315200 \\ \mathrm{H} & -5.45503000 & 0.25268300 & -1.66519200 \\ \mathrm{H} & -3.91924300 & -0.39914900 & -2.28488000 \\ \mathrm{C} & -5.07150000 & -0.69761100 & 0.88272200 \\ \mathrm{H} & -4.53623400 & -0.67445500 & 1.82647900 \\ \mathrm{H} & -5.83175500 & 0.09251900 & 0.87747600 \\ \mathrm{H} & -5.58119800 & -1.66174400 & 0.77506000\end{array}$


<smiles>CN(C)c1ncc(N([O-])c2ccccc2)cn1</smiles>

\section{CBS-QB3 Enthalpy $=-757.959498$ CBS-QB3 Free Energy= -758.020688}

$\begin{array}{lrrr}\mathrm{O}^{2} & & & \\ \mathrm{C} & 4.70455000 & -1.32450400 & 0.22674100 \\ \mathrm{C} & 4.76610100 & -0.07944500 & -0.40077200 \\ \mathrm{C} & 3.63194900 & 0.71315100 & -0.51155900 \\ \mathrm{C} & 2.41295300 & 0.25631600 & 0.00605600 \\ \mathrm{C} & 2.34721000 & -0.98544800 & 0.65178300 \\ \mathrm{C} & 3.49334100 & -1.76720200 & 0.75490500 \\ \mathrm{H} & 5.59300200 & -1.93925700 & 0.31003100 \\ \mathrm{H} & 5.70523700 & 0.27685800 & -0.80855400 \\ \mathrm{H} & 3.66041100 & 1.68660400 & -0.98066400 \\ \mathrm{H} & 1.42112900 & -1.32454800 & 1.09638400 \\ \mathrm{H} & 3.43861100 & -2.72312900 & 1.26316900 \\ \mathrm{C} & -0.06011900 & 0.64998400 & -0.06124200 \\ \mathrm{C} & -1.06334300 & 1.48605200 & 0.44654800 \\ \mathrm{C} & -0.49667400 & -0.56372600 & -0.59943400 \\ \mathrm{H} & -0.79859300 & 2.46582900 & 0.82935800 \\ \mathrm{H} & 0.20113900 & -1.25198900 & -1.06672500 \\ \mathrm{C} & -2.65651900 & -0.07403400 & -0.03872800 \\ \mathrm{~N} & 1.28101100 & 1.11124000 & -0.10297200 \\ \mathrm{O} & 1.47118000 & 2.37357500 & -0.23505200 \\ \mathrm{~N} & -3.96443800 & -0.45526200 & 0.00612200 \\ \mathrm{~N} & -2.33810700 & 1.14060000 & 0.46719500 \\ \mathrm{~N} & -1.76884000 & -0.93208700 & -0.58948100 \\ \mathrm{C} & -4.41545100 & -1.72334800 & -0.54348800 \\ \mathrm{H} & -4.95837300 & -2.29041500 & 0.22085900 \\ \mathrm{H} & -5.09356500 & -1.55464300 & -1.38888300 \\ \mathrm{H} & -3.55926300 & -2.29803800 & -0.88191900 \\ \mathrm{C} & -5.00208500 & 0.41437100 & 0.53692200 \\ \mathrm{H} & -4.55021900 & 1.31325900 & 0.94386400 \\ \mathrm{H} & -5.70951700 & 0.69154200 & -0.25403700 \\ \mathrm{H} & -5.55829200 & -0.10511400 & 1.32508300\end{array}$<smiles>COC1C=CC(=Nc2cnc(N(C)C)nc2)C=C1</smiles>

CBS-QB3 Enthalpy $=-872.850164$ CBS-QB3 Free Energy= -872.919562
01
C
C
C
C
$\mathrm{H}$
3.84905200
2.31892700
1.30716600
1.74617600
2.08252900
-0.04899900
1.59433100
0.86915200
-0.33320200
2.57015900

0.03026500

0.42065300

$-0.23313400$

$-0.81128000$

0.83731200 


$\begin{array}{lrrr}\text { H } & 1.07468500 & -0.92134600 & -1.43192700 \\ \mathrm{C} & -1.06164800 & 0.79088000 & -0.30497700 \\ \mathrm{C} & -1.23247600 & -0.60583700 & 0.12400900 \\ \mathrm{C} & -2.29398200 & 1.52637900 & -0.61031600 \\ \mathrm{C} & -2.42916600 & -1.20526100 & 0.11455500 \\ \mathrm{H} & -0.36795600 & -1.13578600 & 0.50368600 \\ \mathrm{C} & -3.48996700 & 0.93085500 & -0.65986300 \\ \mathrm{H} & -2.17010700 & 2.58351500 & -0.81520700 \\ \mathrm{H} & -2.55422900 & -2.21695700 & 0.48503800 \\ \mathrm{H} & -4.38901700 & 1.48382500 & -0.90562000 \\ \mathrm{C} & -3.66858100 & -0.53758400 & -0.40025300 \\ \mathrm{H} & -3.99811700 & -1.04239700 & -1.32233200 \\ \mathrm{~N} & 0.05068800 & 1.44779600 & -0.38065400 \\ \mathrm{O} & -4.68350000 & -0.79177800 & 0.58705000 \\ \mathrm{O} & -5.96047800 & -0.56602000 & -0.09652700 \\ \mathrm{C} & -6.88765300 & -0.28361300 & 0.93412200 \\ \mathrm{H} & -7.84039500 & -0.15091100 & 0.41670500 \\ \mathrm{H} & -6.96391000 & -1.11999100 & 1.63637400 \\ \mathrm{H} & -6.62104600 & 0.63194800 & 1.47152000 \\ \mathrm{~N} & 5.11374600 & -0.53494300 & 0.20833400 \\ \mathrm{~N} & 2.98522400 & -0.79033300 & -0.69413800 \\ \mathrm{~N} & 3.55571400 & 1.15386200 & 0.57485600 \\ \mathrm{C} & 5.53566000 & -1.80456000 & -0.35717600 \\ \mathrm{H} & 6.40257600 & -1.65723900 & -1.01221600 \\ \mathrm{H} & 5.82626100 & -2.50064000 & 0.43919000 \\ \mathrm{H} & 4.72035500 & -2.23495700 & -0.93019400 \\ \mathrm{C} & 6.11818500 & 0.19253700 & 0.96489600 \\ \mathrm{H} & 6.97640400 & 0.43421300 & 0.32610500 \\ \mathrm{H} & 5.68745600 & 1.11210500 & 1.34837200 \\ \mathrm{H} & 6.47857500 & -0.41829500 & 1.80122800 \\ & & & \\ & & & \end{array}$<smiles>CN(C)c1ncc(N=C2C=CC([O])C=C2)cn1</smiles>

\section{CBS-QB3 Enthalpy= -757.913901 CBS-QB3 Free Energy= -757.975659}

$\begin{array}{lrrr}0{ }^{2} & & \\ \text { C } & 2.95150200 & -0.01381200 & -0.02767600 \\ \text { C } & 1.39802700 & 1.61742100 & 0.31579400 \\ \text { C } & 0.36774600 & 0.80510600 & -0.19270600 \\ \text { C } & 0.80709200 & -0.43476800 & -0.68764900 \\ \text { H } & 1.15715700 & 2.62206200 & 0.65398900 \\ \text { H } & 0.11703600 & -1.09596100 & -1.20569000 \\ \text { C } & -1.99933900 & 0.65920200 & -0.12818600 \\ \text { C } & -2.11179800 & -0.71149300 & 0.39379400 \\ \text { C } & -3.26138200 & 1.35125100 & -0.41339900 \\ \text { C } & -3.28879000 & -1.34564300 & 0.46235500 \\ \text { H } & -1.22020300 & -1.18311500 & 0.78894800 \\ \text { C } & -4.44629000 & 0.73224100 & -0.37862700 \\ \text { H } & -3.17268000 & 2.40087400 & -0.66935900 \\ \text { H } & -3.38150900 & -2.33292800 & 0.90215900 \\ \text { H } & -5.37314700 & 1.25038000 & -0.59788400 \\ \text { C } & -4.57295500 & -0.73225500 & -0.03832500\end{array}$




$\begin{array}{lrrr}\mathrm{H} & -4.81948300 & -1.25899800 & -1.00145500 \\ \mathrm{~N} & -0.91056900 & 1.33779200 & -0.30230800 \\ \mathrm{O} & -5.67953700 & -1.06209700 & 0.69466600 \\ \mathrm{~N} & 4.23797200 & -0.44914700 & 0.11306100 \\ \mathrm{~N} & 2.06579600 & -0.84347400 & -0.61857000 \\ \mathrm{~N} & 2.65616700 & 1.22847000 & 0.42030400 \\ \mathrm{C} & 4.67267200 & -1.73614400 & -0.40301100 \\ \mathrm{H} & 5.39218600 & -1.60027700 & -1.22037900 \\ \mathrm{H} & 5.16336900 & -2.31096200 & 0.39018400 \\ \mathrm{H} & 3.81394400 & -2.28761000 & -0.77252900 \\ \mathrm{C} & 5.27786800 & 0.39881000 & 0.67170800 \\ \mathrm{H} & 6.00591200 & 0.67799700 & -0.10060500 \\ \mathrm{H} & 4.83129500 & 1.29971900 & 1.08042100 \\ \mathrm{H} & 5.81075000 & -0.13939600 & 1.46304500\end{array}$

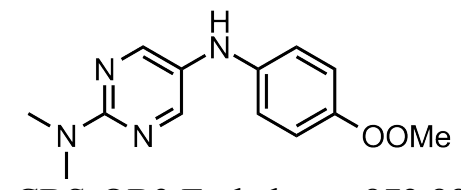

\section{CBS-QB3 Enthalpy $=-872.887074$ CBS-QB3 Free Energy= -872.959967}

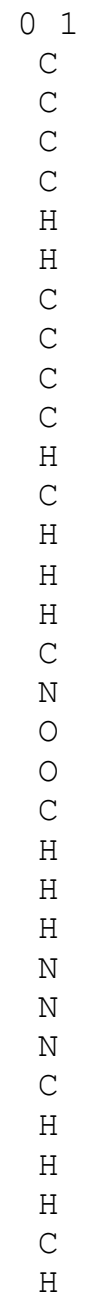

$$
\begin{array}{r}
3.77591400 \\
2.28923200 \\
1.34801800 \\
1.75904500 \\
2.05830500 \\
1.09521700 \\
-1.14352500 \\
-1.27680000 \\
-2.30723000 \\
-2.53598500 \\
-0.39535800 \\
-3.55766300 \\
-2.21698300 \\
-2.64690100 \\
-4.45380900 \\
-3.67980300 \\
0.09423400 \\
-4.90874800 \\
-5.52763300 \\
-6.81287500 \\
-7.26087100 \\
-6.76370400 \\
-7.41830900 \\
4.99419500 \\
2.93870800 \\
3.49101300 \\
5.40985400 \\
6.25767500 \\
5.72547900 \\
4.58274300 \\
5.97940500 \\
6.87021100
\end{array}
$$
$-0.02647500$
0.53527300
$-0.36964600$
$-1.09391200$
1.13655900
$-1.83283200$
$-0.23231500$
0.37131500
$-0.50121000$
0.68075300
0.60232500
$-0.17604900$
$-0.97024300$
1.14310400
$-0.37987100$
0.42428200
$-0.57858400$
0.80358000
$-0.41153600$
0.01847100
$-0.85438400$
0.84737100
0.31085000
0.15802500
$-0.92884200$
0.70422200
$-0.60369300$
$-1.25476700$
0.07727500
$-1.21313700$
1.07671600
0.53073600 


$\begin{array}{rrrr}\mathrm{H} & 5.55073000 & 0.62298800 & 1.61130200 \\ \mathrm{H} & 6.28895000 & -0.98721400 & 1.79398300 \\ \mathrm{H} & 0.10510300 & 2.56831900 & -0.78942300\end{array}$<smiles>CN(C)c1ncc(Nc2ccc([O-])cc2)cn1</smiles>

CBS-QB3 Enthalpy= -757.999698 CBS-QB3 Free Energy= - 758.062087

$$
\begin{array}{rr}
2.93056100 & 0.01728000 \\
1.33111500 & -1.51153900 \\
0.37857700 & -0.84333800 \\
0.84414500 & 0.29582700 \\
1.05674400 & -2.41281800 \\
0.17699800 & 0.86750000 \\
-2.10998900 & -0.63726600 \\
-2.12990800 & 0.64493400 \\
-3.34166400 & -1.23838100 \\
-3.31616100 & 1.29786300 \\
-1.19809100 & 1.09610800 \\
-4.53070600 & -0.59618700 \\
-3.32170000 & -2.22131600 \\
-3.34238200 & 2.27301100 \\
-5.47196400 & -1.04830200 \\
-4.59460100 & 0.72395700 \\
-0.94116000 & -1.33079900 \\
-5.67537400 & 1.32004800 \\
4.21137200 & 0.46350300 \\
2.08684100 & 0.73567000 \\
2.58891700 & -1.11227600 \\
4.68660500 & 1.64829800 \\
5.46867300 & 1.37926100 \\
5.11317300 & 2.35800100 \\
3.86144800 & 2.11729900 \\
5.20202900 & -0.25001800 \\
6.00801000 & -0.62383200 \\
4.73108900 & -1.08680000 \\
5.64303500 & 0.42425700 \\
-1.04384200 & -2.28402200
\end{array}
$$

0.02839300

$-0.52529900$

0.24770100

0.91376400

$-1.07058400$

1.55444500

0.16345700

$-0.44474700$

0.54210600

$-0.64971600$

$-0.76300400$

0.33903600

1.00542000

$-1.12201000$

0.62892800

$-0.26680500$

0.38367300

$-0.45074200$

$-0.09620800$

0.80644400

$-0.63237700$

0.60114100

1.32124000

$-0.11617700$

1.12731300

$-0.88654900$

$-0.24352600$

$-1.39230800$

$-1.62874900$

0.69934000<smiles>CN(C)c1ncc(N=C2C=CC(=O)C=C2)cn1</smiles>

CBS-QB3 Enthalpy= -757.405082

CBS-QB3 Free Energy= -757.464883

01

C
2.92003800

0.01418600

0.05240000 


$\begin{array}{lrrr}\mathrm{C} & 1.37911900 & -1.63703800 & -0.23946000 \\ \mathrm{C} & 0.32753900 & -0.78616400 & 0.16293200 \\ \mathrm{C} & 0.75423400 & 0.48353900 & 0.60429200 \\ \mathrm{H} & 1.14460900 & -2.66141600 & -0.51709200 \\ \mathrm{H} & 0.05185100 & 1.17038700 & 1.06879200 \\ \mathrm{C} & -2.04609600 & -0.64226400 & 0.05480300 \\ \mathrm{C} & -2.17097000 & 0.73005300 & -0.44115300 \\ \mathrm{C} & -3.28716300 & -1.35496400 & 0.35128500 \\ \mathrm{C} & -3.37237800 & 1.32954500 & -0.53623000 \\ \mathrm{H} & -1.27928400 & 1.24044600 & -0.78344000 \\ \mathrm{C} & -4.48965100 & -0.75506300 & 0.28659500 \\ \mathrm{H} & -3.17181700 & -2.38574800 & 0.66630200 \\ \mathrm{H} & -3.48325300 & 2.33229600 & -0.93361400 \\ \mathrm{H} & -5.40807600 & -1.27145500 & 0.54066700 \\ \mathrm{C} & -4.62564600 & 0.64912700 & -0.14250200 \\ \mathrm{~N} & -0.94417600 & -1.31418800 & 0.22791600 \\ \mathrm{O} & -5.70968500 & 1.21483600 & -0.20241900 \\ \mathrm{~N} & 4.20637200 & 0.43865200 & -0.06853400 \\ \mathrm{~N} & 2.01429000 & 0.87980900 & 0.56182100 \\ \mathrm{~N} & 2.63889500 & -1.25859300 & -0.32000500 \\ \mathrm{C} & 4.62072100 & 1.77076400 & 0.34424400 \\ \mathrm{H} & 5.39088900 & 1.70088100 & 1.12084500 \\ \mathrm{H} & 5.04294900 & 2.31491800 & -0.50793700 \\ \mathrm{H} & 3.76500100 & 2.31385900 & 0.73206200 \\ \mathrm{C} & 5.25969300 & -0.42807200 & -0.57496900 \\ \mathrm{H} & 6.00152700 & -0.62308000 & 0.20840500 \\ \mathrm{H} & 4.83023800 & -1.36909000 & -0.90302300 \\ \mathrm{H} & 5.76856500 & 0.05766900 & -1.41442300 \\ & & & \\ & & & \end{array}$<smiles>COC1C=CC=CC1=Nc1cnc(N(C)C)nc1</smiles>

\section{CBS-QB3 Enthalpy $=-872.842164$ CBS-QB3 Free Energy= -872.911400}

$\begin{array}{lrrr}01 & & \\ \mathrm{C} & -3.21108100 & 0.05037100 & 0.01605600 \\ \mathrm{C} & -1.31884900 & 1.04936700 & -0.77279800 \\ \mathrm{C} & -0.50356800 & 0.24430400 & 0.03817500 \\ \mathrm{C} & -1.21060300 & -0.62110900 & 0.88558500 \\ \mathrm{H} & -0.85912800 & 1.78814400 & -1.42463200 \\ \mathrm{H} & -0.67873300 & -1.22588600 & 1.61652700 \\ \mathrm{~N} & 0.87688000 & 0.45193600 & 0.06236200 \\ \mathrm{C} & 1.74103500 & -0.49919900 & 0.03374900 \\ \mathrm{C} & 1.46987900 & -1.90892200 & -0.23479400 \\ \mathrm{C} & 2.46880300 & -2.79042400 & -0.46127800 \\ \mathrm{H} & 0.44079200 & -2.21336100 & -0.37456300 \\ \mathrm{C} & 4.22148300 & -1.14248400 & -0.13901000 \\ \mathrm{C} & 3.86885500 & -2.40083900 & -0.43196400 \\ \mathrm{H} & 2.22592500 & -3.81104300 & -0.73814100 \\ \mathrm{H} & 5.25302300 & -0.81093600 & -0.15193300 \\ \mathrm{H} & 4.62032500 & -3.13924200 & -0.68782100 \\ \mathrm{C} & 3.21278500 & -0.11440800 & 0.28599400\end{array}$




$\mathrm{H}$
$\mathrm{O}$
$\mathrm{O}$
$\mathrm{C}$
$\mathrm{H}$
$\mathrm{H}$
$\mathrm{H}$
$\mathrm{N}$
$\mathrm{N}$
$\mathrm{N}$
$\mathrm{C}$
$\mathrm{H}$
$\mathrm{H}$
$\mathrm{H}$
$\mathrm{C}$
$\mathrm{H}$
$\mathrm{H}$
$\mathrm{H}$

$$
\begin{array}{r}
3.31013500 \\
3.58651200 \\
3.47193500 \\
2.74376900 \\
1.73657300 \\
2.70670100 \\
3.27027900 \\
-4.57140400 \\
-2.63833600 \\
-2.53305200 \\
-5.28682300 \\
-5.93874000 \\
-5.91518200 \\
-4.57526700 \\
-5.39199400 \\
-6.02473400 \\
-4.75296600 \\
-6.04733800
\end{array}
$$

0.00220000

1. 12943100

2.15610600

3.22863500

2.90809000

3.97998300

3. 64051800

$-0.08291500$

0.95612000

$-0.72214900$

$-0.97925400$

$-0.41136400$

$-1.66478600$

$-1.55081100$

0.74175500

1. 41768500

1.33110300

0.10769700
1.37609500

$-0.29584200$

0.73955300

0.16315600

$-0.10810200$

0.95624100

$-0.70405600$

$-0.03297400$

$-0.80771400$

0.88960300

0.85818400

1. 53428200

0.27794900

1. 44583800

$-0.90271100$

$-0.31301200$

$-1.55283100$

$-1.51052700$<smiles>CN(C)c1ncc(N=C2C=CC=CC2[O])cn1</smiles>

CBS-QB3 Enthalpy= -757.907404

CBS-QB3 Free Energy= -757.970175

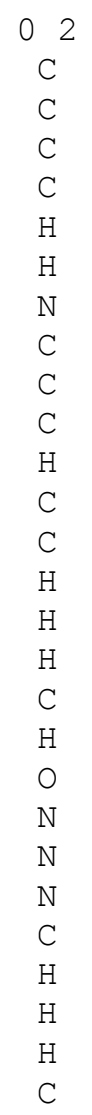

$$
\begin{array}{r}
-2.83272900 \\
-1.12044500 \\
-0.17831600 \\
-0.73726700 \\
-0.77801400 \\
-0.11337700 \\
1.15326400 \\
2.16102200 \\
2.15074900 \\
3.28054100 \\
1.21798800 \\
4.71034300 \\
4.56752400 \\
3.24696700 \\
5.67299900 \\
5.42367800 \\
3.54518200 \\
3.47389800 \\
3.80203200 \\
-4.15949000 \\
-2.41341500 \\
-2.03312700 \\
-4.72230600 \\
-5.42649100 \\
-5.26708700 \\
-3.92433500 \\
-5.10816200
\end{array}
$$

$-0.05473100$

0.12746000

$-0.26028900$

$-0.61103200$

0.35154500

$-1.02645600$

$-0.40208400$

$-0.09798000$

0.55487300

0.68239500

0.97485000

$-0.32681200$

0.18488900

1.19349600

$-0.64015000$

0.27081900

$-0.39412300$

$-1.42733700$

0.34422600

0.10751400

0.25118600

$-0.52293900$

$-0.26000600$

$-1.09422700$

0.58927600

$-0.55481000$

0.55154500 


$\begin{array}{llrr}\mathrm{H} & -5.80641500 & -1.01608800 & -0.25522200 \\ \mathrm{H} & -4.57274000 & -1.65299100 & 0.85558500 \\ \mathrm{H} & -5.69075300 & -0.37464300 & 1.39594900\end{array}$<smiles>COc1ccccc1Nc1cnc(N(C)C)nc1</smiles>

CBS-QB3 Enthalpy $=-872.891601$ CBS-QB3 Free Energy= -872.961154

01

C

C

C

C

$\mathrm{H}$

$\mathrm{H}$

$\mathrm{N}$

C

C

C

$\mathrm{H}$

C

C

$\mathrm{H}$

O

O

C

$\mathrm{H}$

$\mathrm{H}$

$\mathrm{H}$

N

$\mathrm{N}$

C

$\mathrm{H}$

$\mathrm{H}$

$\mathrm{H}$

$\mathrm{H}$

$\mathrm{H}$

H

\begin{abstract}
3.27409700
1.33796200

0.57949800

1. 32026600

0.84895100

0.81434700

$-0.82052000$

$-1.74409300$

$-1.40700900$

$-2.39977200$

$-0.36680900$

$-4.10331400$

$-3.75172500$

$-2.10876200$

$-5.13830500$

$-4.51517700$

$-3.11897300$

$-3.48578600$

$-3.23259200$

$-4.01844100$

$-3.72933800$

$-3.81009800$

$-5.08523100$

4.63327300

2.65622500

2. 64252700

5.39459100

6.05539200

6.01826900

4.71381600

5.40890400

6.06793600

4.73796700

6.03541300

$-1.20382200$
\end{abstract}

0.13308500

$-0.58996700$

0.28788600

1.09035800

$-1.23668200$

1.81400700

0.40773600

$-0.58024100$

$-1.86728500$

$-2.80417400$

$-2.12732300$

$-1.21697400$

$-2.49487200$

$-3.79109900$

$-0.92411800$

$-3.23265800$

$-0.28158900$

0.92300500

2.01058600

3.10107900

3.43665900

3.89362100

2.85629700

0.03780900

$-0.68888900$

1.04022700

0.90560300

1.56222100

0.30454800

1.51402900

$-0.87288400$

$-0.31464100$

$-1.49243300$

$-1.51144900$

1.34197000
0.03033400

0.99887000

0.21842700

$-0.65199200$

1.72348800

$-1.28865200$

0.34491600

0.05467900

$-0.39232500$

$-0.65947500$

$-0.53997000$

$-0.08447500$

$-0.50528100$

$-1.00221600$

0.04615800

$-0.71875400$

0.21973800

0.75115100

$-0.29999300$

0.13043000

1.13212000

$-0.59389500$

0.11243700

$-0.07948400$

0.90794100

$-0.74485200$

$-0.96139000$

$-0.38107300$

$-1.63292700$

$-1.54836900$

0.74492700

1.42206900

1.33160200

0.11173900

0.36252500<smiles>CN(C)c1ncc(Nc2ccccc2[O-])cn1</smiles>

CBS-QB3 Enthalpy $=-758.009057$ 


\section{CBS-QB3 Free Energy= -758.070949}

$\begin{array}{lrrr}\text { O } 2 & & \\ \mathrm{C} & -2.84534600 & -0.01322000 & 0.02449300 \\ \mathrm{C} & -0.73384000 & 0.67081700 & 0.57095300 \\ \mathrm{C} & -0.18876500 & -0.52741100 & 0.09255500 \\ \mathrm{C} & -1.12632400 & -1.45322400 & -0.38529300 \\ \mathrm{H} & -0.09983600 & 1.43065400 & 1.01690000 \\ \mathrm{H} & -0.79425300 & -2.42432800 & -0.74840100 \\ \mathrm{~N} & 1.16947600 & -0.85875100 & 0.13209300 \\ \mathrm{C} & 2.27638500 & -0.08729400 & -0.01854900 \\ \mathrm{C} & 2.31727100 & 1.26529200 & -0.38968200 \\ \mathrm{C} & 3.53900400 & 1.90241600 & -0.51873700 \\ \mathrm{H} & 1.40373100 & 1.80561900 & -0.60099900 \\ \mathrm{C} & 4.76089200 & -0.10734000 & 0.06772900 \\ \mathrm{C} & 4.75822800 & 1.21688000 & -0.28002700 \\ \mathrm{H} & 3.56453200 & 2.94398100 & -0.81712600 \\ \mathrm{H} & 5.67845400 & -0.65582300 & 0.24325000 \\ \mathrm{H} & 5.69461700 & 1.75337300 & -0.38521500 \\ \mathrm{C} & 3.53045300 & -0.84301000 & 0.21354400 \\ \mathrm{O} & 3.46709500 & -2.05354000 & 0.51395500 \\ \mathrm{~N} & -4.17957700 & 0.26144100 & -0.04478100 \\ \mathrm{~N} & -2.03080600 & 0.93617800 & 0.53424800 \\ \mathrm{~N} & -2.42660300 & -1.22116300 & -0.41766200 \\ \mathrm{C} & -5.14132600 & -0.71150000 & -0.53718100 \\ \mathrm{H} & -5.82702500 & -1.01235700 & 0.26437300 \\ \mathrm{H} & -5.73509000 & -0.27292200 & -1.34673600 \\ \mathrm{H} & -4.61747900 & -1.58745600 & -0.90626000 \\ \mathrm{C} & -4.73313900 & 1.51301000 & 0.44568000 \\ \mathrm{H} & -5.38828300 & 1.33228400 & 1.30675700 \\ \mathrm{H} & -3.92711600 & 2.17566800 & 0.74433200 \\ \mathrm{H} & -5.32793400 & 1.99130300 & -0.34025500 \\ \mathrm{H} & 1.43054100 & -1.82810900 & 0.31754600\end{array}$<smiles>CN(C)c1ncc(N=C2C=CC=CC2=O)cn1</smiles>

\section{CBS-QB3 Enthalpy= -757.410032 CBS-QB3 Free Energy= -757.469994}

$$
\begin{array}{cc}
\mathrm{O}^{1} & 1 \\
\mathrm{C} \\
\mathrm{C} \\
\mathrm{C} \\
\mathrm{C} \\
\mathrm{C} \\
\mathrm{C} \\
\mathrm{H} \\
\mathrm{H} \\
\mathrm{H} \\
\mathrm{H} \\
\mathrm{H}
\end{array}
$$

$$
\begin{aligned}
& -4.80250700 \\
& -4.74995700 \\
& -3.59075100 \\
& -2.43803900 \\
& -2.50216000 \\
& -3.68174900 \\
& -5.71835700 \\
& -5.62499700 \\
& -3.54150200 \\
& -1.64443200 \\
& -3.72562900
\end{aligned}
$$
0.11789200
$-0.57009400$
$-0.56141200$
0.09032300
0.79683900
0.81279500
0.13036200
$-1.09744600$
$-1.06206000$
1.37217200
1.37541500 


$\begin{array}{lrrr}\mathrm{N} & -1.31341400 & 1.18287600 & 0.08776300 \\ \mathrm{C} & -0.11937800 & 0.75448700 & -0.10107300 \\ \mathrm{C} & 0.24472600 & -0.58950200 & -0.57572700 \\ \mathrm{H} & -0.53629900 & -1.22976500 & -0.98205300 \\ \mathrm{C} & 2.44757200 & -0.14319200 & -0.12813800 \\ \mathrm{C} & 1.05344800 & 1.71074400 & 0.09510500 \\ \mathrm{O} & 0.87430800 & 2.90134000 & 0.22549600 \\ \mathrm{~N} & 3.66564100 & -0.72394500 & -0.00222200 \\ \mathrm{~N} & 2.30204000 & 1.12514300 & 0.15559300 \\ \mathrm{~N} & 1.45113500 & -1.02123100 & -0.62140700 \\ \mathrm{C} & 4.80207800 & 0.04751400 & 0.48654800 \\ \mathrm{H} & 5.24803400 & -0.46021700 & 1.34757700 \\ \mathrm{H} & 5.56185300 & 0.13011600 & -0.29822300 \\ \mathrm{H} & 4.46265000 & 1.03931300 & 0.76851500 \\ \mathrm{C} & 3.95088800 & -2.10883100 & -0.35692500 \\ \mathrm{H} & 3.06189800 & -2.57882300 & -0.76198500 \\ \mathrm{H} & 4.75447700 & -2.13853800 & -1.10025400 \\ \mathrm{H} & 4.28500300 & -2.65944200 & 0.52912200\end{array}$

